\title{
HiggsSignals: Confronting arbitrary Higgs sectors with measurements at the Tevatron and the LHC
}

\author{
Philip Bechtle ${ }^{1, \mathrm{a}}$, Sven Heinemeyer ${ }^{2, \mathrm{~b}}$, Oscar Stål ${ }^{3, \mathrm{c}}$, Tim Stefaniak ${ }^{1,4, \mathrm{~d}}$, Georg Weiglein ${ }^{5, \mathrm{e}}$ \\ ${ }^{1}$ Physikalisches Institut der Universität Bonn, Nußallee 12, 53115 Bonn, Germany \\ ${ }^{2}$ Instituto de Física de Cantabria (CSIC-UC), Santander, Spain \\ 3 The Oskar Klein Centre, Department of Physics, Stockholm University, 10691 Stockholm, Sweden \\ ${ }^{4}$ Bethe Center for Theoretical Physics, University of Bonn, Nußallee 12, 53115 Bonn, Germany \\ ${ }^{5}$ Deutsches Elektronen-Synchrotron DESY, Notkestrasse 85, 22607 Hamburg, Germany
}

Received: 10 May 2013 / Accepted: 6 December 2013 / Published online: 7 February 2014

(C) The Author(s) 2014. This article is published with open access at Springerlink.com

\begin{abstract}
HiggsSignals is a Fortran90 computer code that allows to test the compatibility of Higgs sector predictions against Higgs rates and masses measured at the LHC or the Tevatron. Arbitrary models with any number of Higgs bosons can be investigated using a model-independent input scheme based on HiggsBounds. The test is based on the calculation of a $\chi^{2}$ measure from the predictions and the measured Higgs rates and masses, with the ability of fully taking into account systematics and correlations for the signal rate predictions, luminosity and Higgs mass predictions. It features two complementary methods for the test. First, the peak-centered method, in which each observable is defined by a Higgs signal rate measured at a specific hypothetical Higgs mass, corresponding to a tentative Higgs signal. Second, the mass-centered method, where the test is evaluated by comparing the signal rate measurement to the theory prediction at the Higgs mass predicted by the model. The program allows for the simultaneous use of both methods, which is useful in testing models with multiple Higgs bosons. The code automatically combines the signal rates of multiple Higgs bosons if their signals cannot be resolved by the experimental analysis. We compare results obtained with HiggsSignals to official ATLAS and CMS results for various examples of Higgs property determinations and find very good agreement. A few examples of Higgs Signals applications are provided, going beyond the scenarios investigated by the LHC collaborations. For models with more than one Higgs boson we recommend to use HiggsSignals and
\end{abstract}

\footnotetext{
a e-mail: bechtle@physik.uni-bonn.de

b e-mail: Sven.Heinemeyer@cern.ch

c e-mail: oscar.stal@fysik.su.se

d e-mail: tim@th.physik.uni-bonn.de

e e-mail: Georg.Weiglein@desy.de
}

HiggsBounds in parallel to exploit the full constraining power of Higgs search exclusion limits and the measurements of the signal seen at $m_{H} \approx 125.5 \mathrm{GeV}$.

\section{Contents}

1 Introduction . . . . . . . . . . 2

2 Higgs signals in collider searches . . . . . . . 3

3 Statistical approach in HiggsSignals . . . . . . 4

3.1 The peak-centered $\chi^{2}$ method ... . . . . 5 5

3.1.1 Signal strength modifiers . . . . . . . . 6

3.1.2 Higgs mass observables . . . . . . . 7

3.1.3 Assignment of multiple Higgs bosons . . 7

3.2 The mass-centered $\chi^{2}$ method . . . . . . . . 8

3.2.1 Theory mass uncertainties . . . . . . . 9

3.2.2 The Stockholm clustering scheme . . . . 9

3.3 Simultaneous use of both methods . . . . . . . 11

4 Using HiggsSignals . . . . . . . . . . . . . 11

4.1 Installation . . . . . . . . . . . . 11

4.2 Input and output . . . . . . . . . . . 11

4.3 Running HiggsSignals on the command line 14

4.4 Higgs Signals subroutines . . . . . . . 15

4.5 Example programs . . . . . . . . . . . . 19

4.6 Input of new experimental data into Higgs-

Signals . . . . . . . . . . 20

5 Higgs Signals applications . . . . . . . . 21

5.1 Performance studies of HiggsSignals . . . 22

5.1.1 The peak-centered $\chi^{2}$ method for a SM-

like Higgs boson . . . . . . . . . . . 22

5.1.2 Combining search channels with the masscentered $\chi^{2}$ method . . . . . . . . . 25

5.2 Validation with official fit results for Higgs coupling scaling factors . . . . . . . 26 
5.3 Example applications of HiggsSignals . . . 32

6 Conclusions .............. 35

7 Appendix ............... . . 36

7.1 Theory mass uncertainties in the mass-centered $\chi^{2}$ method ............... 36

\section{Introduction}

Searches for a Higgs boson [1-6] have been one of the driving factors behind experimental particle physics over many years. Until recently, results from these searches have always been in the form of exclusion limits, where different Higgs mass hypotheses are rejected at a certain confidence level (usually $95 \%$ ) by the non-observation of any signal. This has been the case for Standard Model (SM) Higgs searches at LEP [7], the Tevatron [8], and (until July 2012) also for the LHC experiments [9, 10]. Limits have also been presented on extended Higgs sectors in theories beyond the SM, where one prominent example are the combined limits on the Higgs sector of the minimal supersymmetric standard model (MSSM) from the LEP experiments [11,12]. To test the predictions of models with arbitrary Higgs sectors consistently against all the available experimental data on Higgs exclusion limits, we have presented the public tool HiggsBounds $[13,14]$, which recently appeared in version 4 . 0 . $0[15,16]$.

With the recent discovery of a new state-compatible with a SM Higgs boson-by the LHC experiments ATLAS [17] and CMS [18], models with extended Higgs sectors are facing new constraints. It is no longer sufficient to test for nonexclusion, but the model predictions must be tested against the measured mass and rates of the observed state, which contains more information. Testing the model predictions of a Higgs sector with an arbitrary number of Higgs bosons against this Higgs signal ${ }^{1}$ (and potentially against other signals of additional Higgs states discovered in the future) is the purpose of a new public computer program, HiggsSignals, which we present here.

HiggsSignals is a Fortran90/2003 code, which evaluates a $\chi^{2}$ measure to provide a quantitative answer to the statistical question of how compatible the Higgs search data (measured signal strengths and masses) is with the model predictions. This $\chi^{2}$ value can be evaluated with two distinct methods, namely the peak-centered and the mass-centered $\chi^{2}$ method. In the peak-centered $\chi^{2}$ method, the (neutral) Higgs signal rates and masses predicted by the model are

\footnotetext{
${ }^{1}$ Here, and in the following, the phrase Higgs signal refers to any hint or observation of a signal in the data of the Tevatron/LHC Higgs searches, regardless of whether in reality this is due to the presence of a Higgs boson. In fact, the user can directly define the Higgs signals, i.e. the signal strength at a given mass peak or as a function of Higgs masses, which should be considered as observables in HiggsSignals, see Sect. 4.6 for more details.
}

tested against the various signal rate measurements published by the experimental collaborations for a fixed Higgs mass hypothesis. This hypothetical Higgs mass is typically motivated by the signal "peak" observed in the channels with high mass resolution, i.e. the searches for $H \rightarrow \gamma \gamma$ and $H \rightarrow Z Z^{(*)} \rightarrow 4 \ell$. In this way, the model is tested at the mass position of the observed peak. In the mass-centered $\chi^{2}$ method on the other hand, HiggsSignals tries to find for every neutral Higgs boson in the model the corresponding signal rate measurements, which are performed under the assumption of a Higgs boson mass equal to the predicted Higgs mass. Thus, the $\chi^{2}$ is evaluated at the model-predicted mass position. For this method to be applicable, the experimental measurements therefore have to be given for a certain mass range.

The input from the user is given in the form of Higgs masses, production cross sections, and decay rates in a format similar to that used in HiggsBounds. The experimental data from Tevatron and LHC Higgs searches is provided with the program, so there is no need for the user to include these values manually. However, it is possible for the user to modify or add to the data at will. Like HiggsBounds, the aim is to always keep HiggsSignals updated with the latest experimental results.

The usefulness of a generic code such as HiggsSignals has become apparent in the last year, given the intense work by theorists to use the new Higgs measurements as constraints on the SM and theories for new physics [1968]. With HiggsSignals, there now exists a public tool that can be used for both model-independent and modeldependent studies of Higgs masses, couplings, rates, etc. in a consistent framework. The $\chi^{2}$ output of HiggsSignals also makes it convenient to use it as direct input to global fits, where a first example application can be found in Ref. [69].

This document serves both as an introduction to the physics and statistical methods used by HiggsSignals and as a technical manual for users of the code. It is organized as follows. Section 2 contains a very brief review of Higgs searches at hadron colliders, focusing on the published data which provides the key experimental input for HiggsSignals and the corresponding theory predictions. In Section 3 we present the HiggsSignals algorithms, including the precise definitions of the two $\chi^{2}$ methods mentioned above. Section 4 provides the technical description (user manual) for how to use the code. We discuss the performance of HiggsSignals and validate with official fit results for Higgs coupling scaling factors from ATLAS and CMS in Sect. 5. Furthermore, we give some examples of fit results, which can be obtained by interpreting all presently available Higgs measurements. We conclude in Sect. 6. In the appendix, details are given on the implementation of theory mass uncertainties in the mass-centered $\chi^{2}$ method. 
(a)

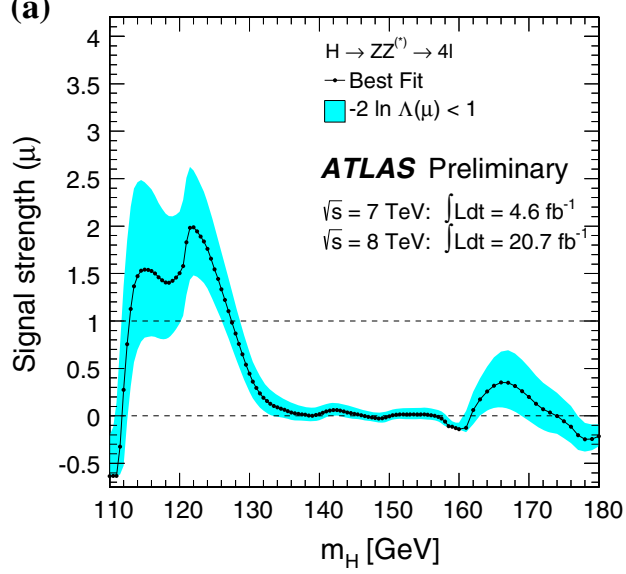

Fig. 1 Measured signal strength modifiers by ATLAS in the search for $H \rightarrow Z Z^{(*)} \rightarrow 4 \ell$ [75] (a) and the best fit rates (in all currently investigated Higgs decay channels) for a Higgs signal at $m_{H}=125.7 \mathrm{GeV}$ according to CMS [77] (b). a The best-fit signal strength $\hat{\mu}$ for the LHC Higgs process $(p p) \rightarrow H \rightarrow Z Z^{(*)} \rightarrow 4 \ell$, given as a function of the

\section{Higgs signals in collider searches}

The experimental data used in Higgs Signals is collected at hadron colliders, mainly the LHC, but there are also some complementary measurements from the Tevatron collider. This will remain the case for the foreseeable future, but the Higgs Signals methods can be easily extended to include data from, for instance, a future $e^{+} e^{-}$linear collider. In this section we give a very brief review of Higgs searches at hadron colliders, focussing the description on the experimental data that provides the basic input for HiggsSignals. For a more complete review see, e.g., Ref. [70-72].

Most searches for Higgs bosons at the LHC are performed under the assumption of the SM. This fixes completely the couplings of the Higgs state to fermions and vector bosons, and both the cross sections and branching ratios are fully specified as a function of the Higgs boson mass, $m_{H}$. Most up-to-date predictions, including an extensive list of references, can be found in $[73,74]$. This allows experiments to measure one-parameter scalings of the total SM rate of a certain (ensemble of) signal channel(s), so-called signal strength modifiers, corresponding to the best fit to the data. These measurements are the basic experimental input used by HiggsSignals. Two examples of this (from ATLAS and CMS) are shown in Fig. 1. The left plot (taken from [75]) shows the measured value of the signal strength modifier, which we denote by $\hat{\mu}$, in the inclusive $p p \rightarrow H \rightarrow Z Z^{(*)} \rightarrow 4 \ell$ process as a function of $m_{H}$ (black line). The cyan band gives a $\pm 1 \sigma$ uncertainty on the measured rate. Since the signal strength modifier is measured relative to its SM value ( $\hat{\mu}=1$, displayed in Fig. 1 by a dashed line), this contains also the theory uncertainties on the SM Higgs cross section (b)

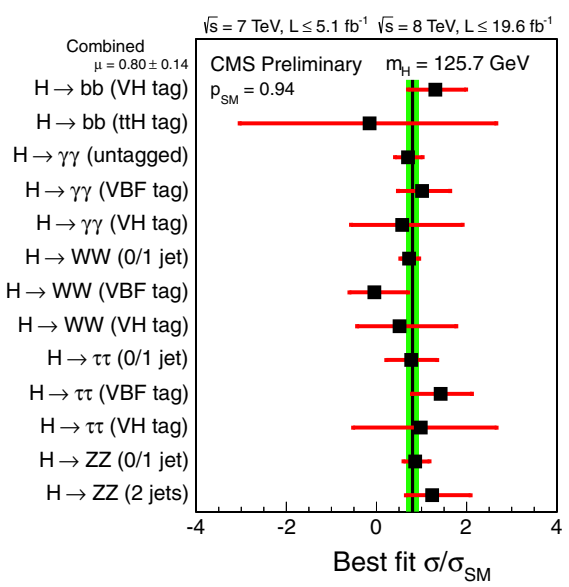

assumed Higgs mass $m_{H}$. The cyan band gives the $68 \%$ C.L. uncertainty of the measurement. b The signal strength of various Higgs channels measured at a fixed hypothetical Higgs mass of $m_{H}=125.7 \mathrm{GeV}$. The combined signal strength scales all Higgs signal rates uniformly and is estimated to $\hat{\mu}_{\mathrm{comb}}=0.80 \pm 0.14$

and branching ratios $[73,74,76]$. As can be seen from Fig. 1, the measured value of $\hat{\mu}$ is allowed to take on negative values. In the absence of sizable signal-background interferenceas is the case for the SM-the signal model would not give $\hat{\mu}<0$. This must therefore be understood as statistical downward fluctuations of the data w.r.t. the background expectation (the average background-only expectation is $\hat{\mu}=0$ ). To keep $\hat{\mu}$ as an unbiased estimator of the true signal strength, it is however essential that the full range of values is retained. As we shall see in more detail below, the applicability of HiggsSignals is limited to the mass range for which measurements of $\hat{\mu}$ are reported. It is therefore highly desirable that experiments publish this information even for mass regions where a SM Higgs signal has been excluded.

A second example of HiggsSignals input, this time from CMS, is shown in the right plot of Fig. 1 (from [77]). This figure summarizes the measured signal strength modifiers for all relevant Higgs decay channels at an interesting value of the Higgs mass, here $m_{H}=125.7 \mathrm{GeV}$. This particular value is typically selected to correspond to the maximal significance for a signal seen in the data. It is important to note that, once a value for $m_{H}$ has been selected, this plot shows a compilation of information for the separate channels that is also available directly from the mass-dependent plots (as shown in Fig. 1a). Again, the error bars on the measured $\hat{\mu}$ values correspond to $1 \sigma$ uncertainties that include both experimental (systematic and statistical) uncertainties, as well as SM theory uncertainties.

The idea of HiggsSignals is to compare the experimental measurements of signal strength modifiers to the Higgs sector predictions in arbitrary models. The model predictions must be provided by the user for each parameter 
point to be tested. To be able to do this consistently, we here describe the basic definitions that we apply. The production of Higgs bosons at hadron colliders can essentially proceed through five partonic subprocesses: gluon fusion (ggf), vector boson fusion (vbf), associated production with a gauge boson $(H W / H Z)$, or associated production with top quarks $(t t H)$, see $[73,74]$ for details. In models with an enhanced Higgs coupling to bottom quarks, the process $b \bar{b} \rightarrow H$ is usually added. In this five-flavor scheme a $b$ quark parton distribution describes the collinear gluon splitting to pairs of bottom quarks inside the proton. This contribution should be matched consistently, and in most cases, added to the gluon fusion subprocess (as prescribed by the Santander matching procedure [78]). We therefore sometimes refer to the sum of the gluon fusion and $b \bar{b} \rightarrow H$ subprocesses as single Higgs production (singleH). Internally, HiggsSignals uses the same LHC cross sections for SM Higgs production at $\sqrt{s}=7$ and $8 \mathrm{TeV}$ as HiggsBounds-4 [15]. The same holds for the reference SM branching ratios, which follow the prescription of the LHC Higgs Cross Section Working Group [73, 74], see also [76] for more details. These branching ratios are the same as those used by the LHC experiments.

The theory prediction for the signal strength modifier of one specific analysis, from a single Higgs boson $H$, is computed in Higgs Signals as

$\mu=\sum_{i} c_{i} \omega_{i}$,

where the sum runs over all channels considered in this analysis. A channel is characterized by one specific production and one specific decay mode. The individual channel signal strength is given by

$c_{i}=\frac{[\sigma \times \mathrm{BR}]_{i}}{\left[\sigma_{\mathrm{SM}} \times \mathrm{BR}_{\mathrm{SM}}\right]_{i}}$,

and the SM channel weight is

$\omega_{i}=\frac{\epsilon_{i}\left[\sigma_{\mathrm{SM}} \times \mathrm{BR}_{\mathrm{SM}}\right]_{i}}{\sum_{j} \epsilon_{j}\left[\sigma_{\mathrm{SM}} \times \mathrm{BR}_{\mathrm{SM}}\right]_{j}}$.

The SM weights contain the relative experimental efficiencies, $\epsilon_{i}$, for the different channels. Unfortunately, these are rarely quoted in experimental publications. If they are available, these numbers can be used by HiggsSignals, which leads to a more reliable comparison between theory predictions and the experimental data for these channels. In the case of unknown efficiencies, all channels considered by the analysis are treated equally, i.e. we set all $\epsilon_{i} \equiv 1$. Note, however, that for many observables approximate numbers for the channel efficiencies can be inferred by reproducing official fit results on scale factors for production cross sections or coupling strengths, which will be further discussed in Sect. 5.2.

One final word of caution should be added here: If the model features a non-standard tensor structure for the par- ticles, which should be confronted with the data, these interactions might lead to observable differences in the experimentally measured kinematic distributions and therefore to changes of the signal acceptance/efficiency of the Higgs analyses. In order to obtain reliable results from HiggsSignals for these types of models, one needs to check whether these effects are negligible. An interface for HiggsSignals, where the user can insert model signal efficiencies for each analysis, which are changed with respect to the SM signal efficiencies, is a planned feature for future development. However, it is impossible to completely unfold this model dependence using only the currently available public information.

\section{Statistical approach in HiggsSignals}

As mentioned already in the introduction, HiggsSignals contains two different statistical methods to test models against the experimental data. These methods are complementary, and to provide a full model test it is advisable in many situations to use both simultaneously. Nevertheless, we leave the final choice of method to the user, and we therefore first describe both methods separately, before discussing their combination in Sect. 3.3.

As already touched upon in the previous section, the search results of ATLAS and CMS are reported in the form of the signal strength modifier $\hat{\mu}$, the ratio of the best-fit signal strength to the expected SM strength of a signal in a certain channel, and its uncertainty $\Delta \hat{\mu}$. In the profile likelihood approach [79] used by the experimental collaborations, $\Delta \hat{\mu}$ is derived from the allowed variation of the signal strength multiplier $\mu$ around the best fit value $\hat{\mu}$. This is calculated using the likelihood ratio $\lambda(\mu)=\mathcal{L}(\mu, \hat{\theta}) / \mathcal{L}(\hat{\mu}, \hat{\theta})$; the ratio of the likelihood function $\mathcal{L}$ for a given $\mu$ with nuisance parameters $\hat{\hat{\theta}}$ optimized at the given value of $\mu$, divided by $\mathcal{L}$ for $\hat{\mu}$ and $\hat{\theta}$ optimized simultaneously (see [79] for more details).

The uncertainty of $\hat{\mu}$ is then calculated using a test statistics based on $-2 \ln \lambda(\mu)$. According to [80,81], this can be expressed as

$-2 \ln \lambda(\mu)=\frac{(\mu-\hat{\mu})^{2}}{\sigma^{2}}+\mathcal{O}(1 / \sqrt{N})$,

where $N$ is the data sample size. Generally, as shown in [79], this converges quite quickly to a central or non-central $\chi^{2}$ distribution, depending on the nuisance parameters. If the test statistics follows a $\chi^{2}$ distribution, the uncertainties of the measurement can generally be treated as Gaussian, hence we interpret all uncertainties $\Delta \hat{\mu}$ as Gaussian, and neglect the $\mathcal{O}(1 / \sqrt{N})$ term. Looking at the experimental results used in HiggsSignals and the available event sample sizes, this 
is justified in almost all analyses, apart from $H \rightarrow Z Z^{*}$, where visible differences from the Gaussian approximation are still possible due to the small event sample size. The largest remaining effects of non-Gaussian distributions are taken into account in HiggsSignals by using asymmetric uncertainties on the measured signal strength in the $\chi^{2}$ calculation, if published as such by the collaborations.

While the $\chi^{2}$ calculated in HiggsSignals can be expected to statistically approximate the true $-2 \ln \lambda$ distribution, cf. Eq. (4), there are three relevant experimental input quantities which can systematically affect the accuracy of the HiggsSignals output in case they are not presented in a complete form in the publicly disclosed information: Firstly, the relative efficiencies $\epsilon_{i}$ of the various Higgs channels/processes considered in the (categories of a) Higgs analysis, as introduced in Eq. (3). Secondly, the correlations of the relevant experimental systematic uncertainties (e.g. of the jet energy scale (JES), $e^{ \pm} / \gamma$ identification and energy scale, tagging efficiencies, etc.) between different Higgs search analyses. Thirdly, the use of continuous variables for classification of channels/production processes (e.g. by using multivariate techniques), which cannot be mapped directly onto signal strengths measurements for distinct categories used as experimental input for the $\chi^{2}$ fit in HiggsSignals. An example for this is the CMS $H \rightarrow Z Z^{*} \rightarrow 4 \ell$ analysis [82]. The effects of such an approach and an approximate solution to this problem within HiggsSignals is discussed in Sect. 5.2.

While the signal efficiencies, $\epsilon_{i}$, could be provided straight-forwardly for every analysis as public information, the communication of the (correlated) systematics, both from experimental and theoretical sources, used in a given analysis is not common. However, within the Gaussian approximation these could in principle be taken into account in HiggsSignals. For the future it would be desirable if this information was provided in a model-independent way. Some ideas on how information on correlated systematic uncertainties in Higgs boson rate measurements could be communicated can be found in Ref. [83]. We discuss the possible impact of including this information in Sect. 5.2 for a few relevant cases.

The $\chi^{2}$ based approach in Higgs Signals could in principle be replaced by the use of likelihood curves from the collaborations, which are currently available in $\left(m_{H}, \hat{\mu}\right)$ grids for a few analyses [77,84], albeit not for the categories individually. Once they are available for the majority of analyses and for every single (category of an) analysis, the $\chi^{2}$ could partly be replaced by the use of these likelihoods. However, significant modifications of the final likelihood by a tool like HiggsSignals would still be required to make it applicable to arbitrary Higgs sectors, due to potentially different signal compositions and hence changed theoretical rate uncertainties. Moreover, the necessity of incorporating cor- related systematics, as mentioned above, remains also in this approach. Already with the currently available statistics the ignorance of efficiencies and correlations of experimental systematics are often the dominant effects for the typically small deviations between the official results by the collaborations and the HiggsSignals results. The assumption on the parabolic shape of the likelihood, on the other hand, has typically a relatively small impact. More details will be given in Sect. 5.2.

\subsection{The peak-centered $\chi^{2}$ method}

The objective of this method is to perform a $\chi^{2}$ test for the hypothesis that a local excess, "signal" (or "peak observable"), in the observed data at a specified mass is generated by the model. In short, this test tries to minimize the total $\chi^{2}$ by assigning, to each Higgs signal in the experimental dataset used, any number of Higgs bosons of the model. From each signal, both the predicted signal strength modifiers and the corresponding predicted Higgs masses (for channels with good mass resolution) enter the total $\chi^{2}$ evaluation in a correlated way. Schematically, the total $\chi^{2}$ is given by

$\chi_{\text {tot }}^{2}=\chi_{\mu}^{2}+\sum_{i=1}^{N_{H}} \chi_{m_{i}}^{2}$,

where $N_{H}$ is the number of (neutral) Higgs bosons of the model. The calculation of the individual contributions from the signal strength modifiers, $\chi_{\mu}^{2}$, and the Higgs masses, $\chi_{m_{i}}^{2}$, will be discussed below.

The input data used in this method is based on the prejudice that a Higgs signal has been observed at a particular Higgs mass value, which does not necessarily have to be the exact same value for all observables. Technically, each observable is defined by a single text file, which contains all relevant information needed by HiggsSignals. An experimental dataset ${ }^{2}$ is then a collection of observables, whose text files are stored in a certain subdirectory of the HiggsSignals distribution. Users may add, modify or remove the experimental data for their own purposes, see Sect. 4.6 for more details.

Currently, an obvious and prominent application of the peak-centered $\chi^{2}$ method would be the test of a single Higgs boson against the rate and mass measurements performed at around $125-126 \mathrm{GeV}$ in all channels reported by the experimental collaborations at the LHC and Tevatron. This scenario will be discussed in detail in Sect. 5. However, HiggsSignals is implemented in a way that is much more general: Firstly, contributions from other Higgs bosons in the

\footnotetext{
2 The most up-to-date experimental data is contained in the folder Expt_tables/latestresults. A summary of these observables, as included in the HiggsSignals-1.0.0 release, is given in Sect. 5, Fig. 2.
} 
model to the Higgs signals will be considered, and if relevant, included in the test automatically. Secondly, the extension of this test to more Higgs signals (in other mass regions) can simply be achieved by the inclusion of the proper experimental data, or for a phenomenological study, the desired pseudo-data.

\subsubsection{Signal strength modifiers}

For $N$ defined signal observables, the total $\chi^{2}$ contribution is given by

$\chi_{\mu}^{2}=\sum_{\alpha=1}^{N} \chi_{\mu, \alpha}^{2}=(\hat{\mu}-\boldsymbol{\mu})^{T} \mathbf{C}_{\mu}^{-1}(\hat{\boldsymbol{\mu}}-\boldsymbol{\mu})$,

where the observed and predicted signal strength modifiers are contained in the $N$-dimensional vectors $\hat{\mu}$ and $\boldsymbol{\mu}$, respectively. $\mathbf{C}_{\mu}$ is the signal strength covariance matrix.

The signal strength covariance matrix $\mathbf{C}_{\mu}$ is constructed in the following way. The diagonal elements $\left(\mathbf{C}_{\mu}\right)_{\alpha \alpha}$ (corresponding to signal observable $\alpha$ ) should first of all contain the intrinsic experimental (statistical and systematic) $1 \sigma$ uncertainties on the signal strengths squared, denoted by $\left(\Delta \hat{\mu}_{\alpha}^{*}\right)^{2}$. These will be treated as uncorrelated uncertainties, since there is no information publicly available on their correlations. We define these uncorrelated uncertainties by subtracting from the total uncertainty $\Delta \hat{\mu}_{\alpha}$ (which is given directly from the $1 \sigma$ error band in the experimental data, cf. Fig. 1) the luminosity uncertainty as well as the theory uncertainties on the predicted signal rate (which we shall include later as correlated uncertainties). Hereby, we assume that these uncertainties can be treated as Gaussian errors. This gives

$$
\left(\Delta \hat{\mu}_{\alpha}^{*}\right)^{2}=\left(\Delta \hat{\mu}_{\alpha}\right)^{2}-\left(\Delta \mathcal{L} \cdot \hat{\mu}_{\alpha}\right)^{2}-\sum_{a=1}^{k}\left(\omega_{a}^{\alpha} \Delta c_{a}^{\mathrm{SM}}\right)^{2} \cdot \hat{\mu}_{\alpha}^{2}
$$

Here, $\Delta \mathcal{L}$ is the relative uncertainty on the luminosity, and $\Delta c_{a}^{\mathrm{SM}}$ is the SM channel rate uncertainty (for a total of $k$ channels contributing to the analysis with signal $\alpha$ ) given by

$\left(\Delta c_{a}^{\mathrm{SM}}\right)^{2}=\left(\Delta \sigma_{a}^{\mathrm{SM}}\right)^{2}+\left(\Delta \mathrm{BR}_{a}^{\mathrm{SM}}\right)^{2}$,

where $\Delta \sigma_{a}^{\mathrm{SM}}$ and $\Delta \mathrm{BR}_{a}^{\mathrm{SM}}$ are the relative systematic uncertainties of the production cross section $\sigma_{a}$ and branching ratio $\mathrm{BR}_{a}$, respectively, of the channel $a$ in the SM. Their values are taken from the LHC Higgs Cross Section Working Group [73,74], evaluated around $m_{H} \sim 125 \mathrm{GeV}$ :

$$
\begin{array}{ll}
\Delta \sigma_{\mathrm{ggf}}^{\mathrm{SM}}=14.7 \%, & \Delta \mathrm{BR}^{\mathrm{SM}}(H \rightarrow \gamma \gamma)=5.4 \%, \\
\Delta \sigma_{\mathrm{VBF}}^{\mathrm{SM}}=2.8 \%, & \Delta \mathrm{BR}^{\mathrm{SM}}(H \rightarrow W W)=4.8 \%, \\
\Delta \sigma_{\mathrm{WH}}^{\mathrm{SM}}=3.7 \%, & \Delta \mathrm{BR}^{\mathrm{SM}}(H \rightarrow Z Z)=4.8 \%, \\
\Delta \sigma_{\mathrm{ZH}}^{\mathrm{SM}}=5.1 \%, & \Delta \mathrm{BR}^{\mathrm{SM}}(H \rightarrow \tau \tau)=6.1 \%, \\
\Delta \sigma_{\mathrm{ttH}}^{\mathrm{SM}}=12.0 \%, & \Delta \mathrm{BR}^{\mathrm{SM}}(H \rightarrow b b)=2.8 \% .
\end{array}
$$

The SM channel weights, $\omega_{a}$, have been defined in (3).

The advantage of extracting $\left(\Delta \hat{\mu}_{\alpha}^{*}\right)^{2}$ via Eq. (7) over using the experimental values $\left(\Delta \hat{\mu}_{\alpha}\right)^{2}$ directly is that it allows for the correlations in the theory uncertainties on the different channel rates to be taken into account. These are correlated to other signals which use the same channels, and since we want to investigate other models beyond the SM, the theory uncertainties on the channel rates are in general different. The same applies for the relative luminosity uncertainties, which can usually be taken equal for all analyses within one collaboration, thus leading to manageable correlations in the signal strength modifiers.

In the next step, we insert these correlated uncertainties into the covariance matrix. To each matrix element $\left(\mathbf{C}_{\mu}\right)_{\alpha \beta}$, including the diagonal, we add a term $\left(\Delta \mathcal{L}_{\alpha} \hat{\mu}_{\alpha}\right)\left(\Delta \mathcal{L}_{\beta} \hat{\mu}_{\beta}\right)$ if the signals $\alpha$ and $\beta$ are observed in analyses from the same collaboration (note that usually the further simplification $\Delta \mathcal{L}_{\alpha}=\Delta \mathcal{L}_{\beta}$ applies in this case). We then add the correlated theory uncertainties of the signal rates, given by

$$
\begin{aligned}
& \left(\sum _ { a = 1 } ^ { k _ { \alpha } } \sum _ { b = 1 } ^ { k _ { \beta } } \left[\delta_{p(a) p(b)} \Delta \sigma_{p(a)}^{\text {model }} \Delta \sigma_{p(b)}^{\text {model }}\right.\right. \\
& \left.\left.\quad+\delta_{d(a) d(b)} \Delta \mathrm{BR}_{d(a)}^{\text {model }} \Delta \mathrm{BR}_{d(b)}^{\text {model }}\right] \cdot \omega_{a, \alpha}^{\text {model }} \omega_{b, \beta}^{\text {model }}\right) \mu_{\alpha} \mu_{\beta} .
\end{aligned}
$$

Here, $k_{\alpha}$ and $k_{\beta}$ are the respective numbers of Higgs (production $\times$ decay) channels considered in the experimental analyses where the signals $\alpha$ and $\beta$ are observed. We use the index notation $p(a)$ and $d(a)$, to map the channel $a$ onto its production and decay processes, respectively. In other words, analyses where the signals share a common production and/or decay mode have correlated systematic uncertainties. These channel rate uncertainties are inserted in the covariance matrix according to their relative contributions to the total signal rate in the model, i.e. via the channel weight evaluated from the model predictions,

$\omega_{i}^{\text {model }}=\frac{\epsilon_{i}[\sigma \times \mathrm{BR}]_{i}}{\sum_{j} \epsilon_{j}[\sigma \times \mathrm{BR}]_{j}}$.

If the theory uncertainties on the Higgs production and decay rates, as well as the channel weights of the model under investigation, are equal to those in the SM, and also the predicted signal strength matches with the observed signal 
strength, the uncertainties $\left(\Delta \hat{\mu}_{\alpha}\right)^{2}$ extracted from the experimental data are exactly restored for the diagonal elements $\left(\mathbf{C}_{\mu}\right)_{\alpha \alpha}$, cf. Eq. (7). Finally, it is worth emphasizing again that this procedure only takes into account the correlations of the luminosity and theoretical signal rate uncertainties, whereas correlations between common experimental uncertainties (energy scale uncertainties, etc.) are neglected. Since this information is not publicly available so far, it could not be included in HiggsSignals.

\subsubsection{Higgs mass observables}

The other type of observables that give contributions to the total $\chi^{2}$ in the peak-centered method is the measured masses corresponding to the observed signals. Not all signals come with a mass measurement; this is something which is specified explicitly in the experimental input data. In general, a Higgs boson in the model that is not assigned to a signal (see below for the precise definition), receives a zero $\chi^{2}$ contribution from this signal. This would be the case, for example, for multiple Higgs bosons that are not close in mass to the observed signal.

HiggsSignals allows the probability density function (pdf) for the Higgs boson masses to be modeled either as a uniform distribution (box), as a Gaussian, or as a box with Gaussian tails. In the Gaussian case, a full correlation in the theory mass uncertainty is taken into account for a Higgs boson that is considered as an explanation for two (or more) signal observables (which include a mass measurement).

Assume that a signal $\alpha$ is observed at the mass $\hat{m}_{\alpha}$, and that a Higgs boson $h_{i}$ with a predicted mass $m_{i}$ (potentially with a theory uncertainty $\Delta m_{i}$ ), is assigned to this signal. Its $\chi^{2}$ contribution is then simply given by

$\chi_{m_{i}, \alpha}^{2}$

$$
=\left\{\begin{array}{ll}
0, & \text { for }\left|m_{i}-\hat{m}_{\alpha}\right| \leq \Delta m_{i}, \\
\infty, & \text { otherwise }
\end{array} \text { with } \quad \Delta m_{i}=\Delta m_{i}+\Delta \hat{m}_{\alpha},\right.
$$

for a uniform (box) mass pdf, and

$$
\begin{aligned}
& \chi_{m_{H, i}, \alpha}^{2} \\
& \quad= \begin{cases}0, & \text { for }\left|m_{i}-\hat{m}_{\alpha}\right| \leq \Delta m_{i}, \\
\left(m_{i}-\Delta m_{i}-\hat{m}_{\alpha}\right)^{2} /\left(\Delta \hat{m}_{\alpha}\right)^{2}, & \text { for } m_{i}-\Delta m_{i}<\hat{m}_{\alpha}, \\
\left(m_{i}+\Delta m_{i}-\hat{m}_{\alpha}\right)^{2} /\left(\Delta \hat{m}_{\alpha}\right)^{2}, & \text { for } m_{i}+\Delta m_{i}>\hat{m}_{\alpha},\end{cases}
\end{aligned}
$$

for a box-shaped pdf with Gaussian tails. Here, we denote the experimental uncertainty of the mass measurement of the analysis associated to signal $\alpha$ by $\Delta \hat{m}_{\alpha}$. The use of a box-shaped mass pdf, Eq. (12), is not recommended in situations where the theory mass uncertainty is small compared to the experimental precision of the mass measurement (and in particular when $\Delta m_{i}=0$ ), since this can lead to overly restrictive results in the assignment of the Higgs boson(s) to high-resolution channels. Moreover, a box-shaped pdf is typically not a good description of the experimental uncertainty of a mass measurement in general. We included this option mostly for illustrational purposes.

In the case of a Gaussian mass pdf the $\chi^{2}$ calculation is performed in a similar way as the calculation of $\chi_{\mu}^{2}$ in Eq. (6). We define for each Higgs boson $h_{i}$

$\chi_{m_{i}}^{2}=\sum_{\alpha=1}^{N} \chi_{m_{i}, \alpha}^{2}=\left(\hat{\mathbf{m}}-\mathbf{m}_{i}\right)^{T} \mathbf{C}_{\mathbf{m}_{i}}^{-1}\left(\hat{\mathbf{m}}-\mathbf{m}_{i}\right)$,

where the $\alpha$ th entry of the predicted mass vector $\mathbf{m}_{i}$ is given by $m_{i}$, if the Higgs boson $h_{i}$ is assigned to the signal $\alpha$, or $\hat{m}_{\alpha}$ otherwise (thus leading to a zero $\chi^{2}$ contribution from this observable and this Higgs boson). As can be seen from Eq. (14), we construct a mass covariance matrix $\mathbf{C}_{m_{i}}$ for each Higgs boson $h_{i}$ in the model. The diagonal elements $\left(\mathbf{C}_{m_{i}}\right)_{\alpha \alpha}$ contain the experimental mass resolution squared, $\left(\Delta \hat{m}_{\alpha}\right)^{2}$, of the analysis in which the signal $\alpha$ is observed. The squared theory mass uncertainty, $\left(\Delta m_{i}\right)^{2}$, enters all matrix elements $\left(\mathbf{C}_{m_{i}}\right)_{\alpha \beta}$ (including the diagonal) where the Higgs boson $h_{i}$ is assigned to both signal observables $\alpha$ and $\beta$. Thus, the theoretical mass uncertainty is treated as fully correlated.

The sign of this correlation depends on the relative position of the predicted Higgs boson mass, $m_{i}$, with respect to the two (different) observed mass values, $\hat{m}_{\alpha, \beta}$ (where we assume $\hat{m}_{\alpha}<\hat{m}_{\beta}$ for the following discussion): If the predicted mass lies outside the two measurements, i.e. $m_{i}<\hat{m}_{\alpha}, \hat{m}_{\beta}$ or $m_{i}>\hat{m}_{\alpha}, \hat{m}_{\beta}$, then the correlation is assumed to be positive. If it lies in between the two mass measurements, $\hat{m}_{\alpha}<m_{i}<\hat{m}_{\beta}$, the correlation is negative (i.e. we have anti-correlated observables). The necessity of this sign dependence can be illustrated as follows: Let us assume the predicted Higgs mass is varied within its theoretical uncertainty. In the first case, the deviations of $m_{i}$ from the theoretical mass uncertainties $\hat{m}_{\alpha, \beta}$ both either increase or decrease (depending on the direction of the mass variation). Thus, the theoretical mass uncertaintines are positively correlated. However, in the latter case, a variation of $m_{i}$ towards one mass measurement always corresponds to a larger deviation of $m_{i}$ from the other mass measurements. Therefore, the theoretical mass uncertainties for these observables have to be anti-correlated.

\subsubsection{Assignment of multiple Higgs bosons}

If a model contains an extended (neutral) Higgs sector, it is a priori not clear which Higgs boson(s) give the best explanation of the experimental observations. Moreover, possible superpositions of the signal strengths of the Higgs bosons have to be taken into account. Another (yet hypothetical) complication arises if more than one Higgs signal has been discovered in the same Higgs search, indicating the 
discovery of another Higgs boson. In this case, care has to be taken that a Higgs boson of the model is only considered as an explanation of one of these signals.

In the peak-centered $\chi^{2}$ method, these complications are taken into account by the automatic assignment of the Higgs bosons in the model to the signal observables. In this procedure, Higgs Signals tests whether the combined signal strength of several Higgs bosons might yield a better fit than the assignment of a single Higgs boson to one signal in an analysis. Moreover, based on the predicted and observed Higgs mass values, as well as their uncertainties, the program decides whether a comparison of the predicted and observed signal rates is valid for the considered Higgs boson. A priori, all possible Higgs combinations which can be assigned to the observed signal(s) of an analysis are considered. If more than one signal exists in one analysis, it is taken care of that each Higgs boson is assigned to at most one signal to avoid double-counting. A signal to which no Higgs boson is assigned contributes a $\chi^{2}$ penalty given by Eq. (6) with the corresponding model prediction $\mu_{\alpha}=0$. This corresponds to the case where an observed signal cannot be explained by any of the Higgs bosons in the model.

For each Higgs search analysis the best Higgs boson assignment is found in the following way: For every possible assignment $\eta$ of a Higgs boson combination to the signal $\alpha$ observed in the analysis, its corresponding tentative $\chi^{2}$ contribution, $\chi_{\alpha, \eta}^{2}$, based on both the signal strength and potentially the Higgs mass measurement, is evaluated. In order to be considered for the assignment, the Higgs combination has to fulfill the following requirements:

- Higgs bosons which have a mass $m_{i}$ close enough to the signal mass $\hat{m}_{\alpha}$, i.e.

$$
\left|m_{i}-\hat{m}_{\alpha}\right| \leq \Lambda \sqrt{\left(\Delta m_{i}\right)^{2}+\left(\Delta \hat{m}_{\alpha}\right)^{2}}
$$

are required to be assigned to the signal $\alpha$. Here, $\Lambda$ denotes the assignment range, which can be modified by the user, see Sect. 4.4 (the default setting is $\Lambda=1$ ).

- If the $\chi^{2}$ contribution from the measured Higgs mass is deactivated for this signal, combinations with a Higgs boson that fulfills Eq. (15) are taken into account for a possible assignment, and not taken into account otherwise.

- If the $\chi^{2}$ contribution from the measured Higgs mass is activated, combinations with a Higgs boson mass which does not fulfill Eq. (15) are still considered. Here, the difference of the measured and predicted Higgs mass is automatically taken into account by the $\chi^{2}$ contribution from the Higgs mass, $\chi_{m}^{2}$.

In the case where multiple Higgs bosons are assigned to the same signal, the combined signal strength modifier $\mu$ is taken as the sum over their predicted signal strength modifiers (corresponding to incoherently adding their rates). The best Higgs-to-signals assignment $\eta_{0}$ in an analysis is that which minimizes the overall $\chi^{2}$ contribution, i.e.

$\eta_{0}=\eta, \quad$ where $\sum_{\alpha=1}^{\mathrm{N}_{\text {signals }}} \chi_{\alpha, \eta}^{2}$ is minimal.

Here, the sum runs over all signals observed within this particular analysis. In this procedure, HiggsSignals only considers assignments $\eta$ where each Higgs boson is not assigned to more than one signal within the same analysis in order to avoid double counting.

There is also the possibility to enforce that a collection of peak observables is either assigned or not assigned in parallel. This can be useful if certain peak observables stem from the same Higgs analysis but correspond to measurements performed for specific tags or categories (e.g. as presently used in $H \rightarrow \gamma \gamma$ analyses). See Sect. 4.6 for a description of these assignment groups.

A final remark should be made on the experimental resolution, $\Delta \hat{m}_{\alpha}$, which enters Eq. (15). In case the analysis has an actual mass measurement that enters the $\chi^{2}$ contribution from the Higgs mass, $\Delta \hat{m}_{\alpha}$ gives the uncertainty of the mass measurement. If this is not the case, $\Delta \hat{m}_{\alpha}$ is an estimate of the mass range in which two Higgs boson signals cannot be resolved. This is taken to be the mass resolution quoted by the experimental analysis. Typical values are, for instance, $10 \%$ (for $V H \rightarrow V(b \bar{b})$ [85]) and 20\% (for $H \rightarrow \tau \tau$ [86] and $H \rightarrow W W^{(*)} \rightarrow \ell v \ell v$ [87]) of the assumed Higgs mass. It should be kept in mind that the HiggsSignals procedure to automatically assign (possibly several) Higgs bosons to the signals potentially introduces sharp transitions from assigned to unassigned signals at certain mass values, see Sect. 5.1.1 for a further discussion. More detailed studies of overlapping signals from multiple Higgs bosons, where possible interference effects are taken into account, are desirable in case evidence for such a scenario emerges in the future data.

\subsection{The mass-centered $\chi^{2}$ method}

The mass-centered $\chi^{2}$ method is complementary to the peakcentered $\chi^{2}$ method, since it allows for a more general test of the model against the experimental data without reference to particular signals. This method uses the data where the measured best-fit signal strength modifiers are published as a function of the Higgs mass over the (full) investigated mass range, as shown in Fig. 1a. ${ }^{3} \mathrm{~A} \chi^{2}$ test can then be performed

\footnotetext{
3 This is sometimes referred to as the "cyan-band plot", or alternatively the " $\hat{\mu}$ plot".
} 
directly at the predicted Higgs mass(es), $m_{i}$, of the model if these fall within the experimentally investigated mass range of an analysis $a$ (denoted by $G_{a}$ ). For Higgs bosons that are outside this mass range, HiggsSignals provides no information. Also in this method, like in the peak-centered case, it can be necessary to consider the combined rates of several Higgs bosons which are close in mass compared to the experimental resolution. We begin with a general discussion of the single Higgs (non-mass-degenerate) case, and outline the combination scheme below.

\subsubsection{Theory mass uncertainties}

In the $\hat{\mu}$ plot the experimental mass uncertainty is already taken into account in the experimental analysis. However, we also want to take into account a possible theoretical uncertainty on the predicted Higgs mass, $\Delta m_{i}$. HiggsSignals provides two different methods to include theoretical Higgs mass uncertainties in the mass-centered $\chi^{2}$ evaluation:

(i) (default setting) In the first method the predicted Higgs mass is varied around $m_{i}$ within its uncertainties. We denote this varied mass by $m^{\prime}$ in the following. For a uniform (box) parametrization of the theoretical mass uncertainty, we have the allowed mass range

$m^{\prime} \in\left[m_{i}-\Delta m_{i}, m_{i}+\Delta m_{i}\right] \equiv M_{i}$.

A tentative $\chi^{2}$ distribution is evaluated as a function of $m^{\prime}$, which, in the uniform (box) parametrization, takes the form

$\chi_{i}^{2}\left(m^{\prime}\right)=\sum_{a=1}^{n} \frac{\left[\mu_{a}\left(m_{i}\right)-\hat{\mu}_{a}\left(m^{\prime}\right)\right]^{2}}{\left(\Delta \hat{\mu}_{a}\left(m^{\prime}\right)\right)^{2}} \quad\left(m^{\prime} \in M_{i}\right)$.

For the Gaussian parametrization, we have

$$
\begin{aligned}
\chi_{i}^{2}\left(m^{\prime}\right)= & \sum_{a=1}^{n}\left(\frac{\left[\mu_{a}\left(m_{i}\right)-\hat{\mu}_{a}\left(m^{\prime}\right)\right]^{2}}{\left(\Delta \hat{\mu}_{a}\left(m^{\prime}\right)\right)^{2}}\right) \\
& +\frac{\left[m_{i}-m^{\prime}\right]^{2}}{\left(\Delta m_{i}\right)^{2}} \text { with } m^{\prime} \in G_{a} .
\end{aligned}
$$

In these expressions, $n$ denotes the total number of considered analyses. Note that the predicted signal strengths, $\mu_{a}$, are always calculated at the predicted central values for the Higgs mass, $m_{i}$, (from the user input), and the signal strength is held fixed in the mass variation. This is clearly an approximation, but for small theory mass uncertainties $\Delta m_{i}$ it is reasonable to treat resulting variations in $\mu$ as a second-order effect. ${ }^{4}$ From a practical viewpoint, it also reduces significantly the amount of model information that has to be supplied by the user. The final values for $\hat{\mu}$ and $\Delta \hat{\mu}$ are chosen for each Higgs boson $h_{i}$ at the mass value $m_{i}^{0}=m^{\prime}$, where $\chi_{i}^{2}\left(m^{\prime}\right)$ is minimized (i.e. for each Higgs boson separately, but combining all channels). In this way, the most conservative value of the predicted Higgs mass, within its theory uncertainty, is used to define the measured signal strength modifiers for the final $\chi^{2}$ evaluation.

(ii) In the second approach to include theory mass uncertainties, Higgs Signals convolves the experimentally measured signal strength modifier, $\hat{\mu}_{a}(m)$, with a theory mass pdf, $g\left(m^{\prime}, m\right)$, resulting in

$\hat{\mu}_{a}^{\text {conv }}(m)=\int_{G_{a}} \mathrm{~d} m^{\prime} \hat{\mu}_{a}\left(m^{\prime}\right) g\left(m^{\prime}, m\right)$.

The theory mass pdf $g\left(m^{\prime}, m\right)$ can again be chosen to be either a uniform (box) distribution or a Gaussian, both centered around the predicted mass value, $m$, and with a box width of $\pm \Delta m$ or a Gaussian width $\Delta m$, respectively. The pdf is normalized to unity over the mass range $G_{a}$ in order to preserve probability. In the case of zero theoretical Higgs mass uncertainty, ${ }^{5} g\left(m^{\prime}, m\right)=\delta\left(m^{\prime}-m\right)$ in either case. The model prediction is therefore tested directly against the measured value $\hat{\mu}(m)$ at the predicted (exact) value for the mass $m$.

The observed signal strength modifier after convolution, $\hat{\mu}_{a}^{\text {conv }}$, now includes contributions to the measured signal strength modifier from the mass region close to the predicted Higgs mass (weighted by $g\left(m^{\prime}, m\right)$ ). Similarly, the upper and lower experimental $1 \sigma$ uncertainty (cyan) band values, $\Delta \hat{\mu}_{a}$, are smeared

$$
\Delta \hat{\mu}_{a}^{\text {conv }}(m)=\int_{G_{a}} \mathrm{~d} m^{\prime} \Delta \hat{\mu}_{a}\left(m^{\prime}\right) g\left(m^{\prime}, m\right) .
$$

In this case it is the smeared quantities, evaluated from Eqs. (20) and (21), that enter the $\chi^{2}$ test.

\subsubsection{The Stockholm clustering scheme}

If more than one neutral Higgs boson of the model has a mass in the relevant region of an analysis, $m_{i} \in G_{a}$, possible superpositions of their signal rates have to be taken into

\footnotetext{
4 This requirement puts an upper limit on a reasonable theoretical mass uncertainty: it should be smaller than the typical mass interval over which the rate predictions vary significantly (in the relevant channels).

5 This is, e.g., the case in the SM, where the Higgs mass is a free parameter, or in the (low-energy) MSSM, where, for instance, the mass of the pseudoscalar Higgs boson $A$ can be chosen to be an input parameter.
} 
account without double-counting. In order to determine the relevant combinations (out of the potentially many options), we use a prescription inspired by jet clustering. In a similar spirit, we call this the Stockholm clustering scheme:

1. Determine the nearest neighboring Higgs bosons $h_{i}$ and $h_{j}$ by their mass difference $\Delta m_{i j}=\left|m_{i}-m_{j}\right|$. If $\min \left(\Delta m_{i j}\right)$ is larger than the experimental mass resolution of the analysis, the clustering is finished, and we proceed to step 4. If it is smaller, the two Higgs bosons $h_{i}$ and $h_{j}$ will be clustered (combined).

2. The combination of two adjacent Higgs bosons $h_{i}$ and $h_{j}$ defines a new Higgs cluster $h_{k}$ with the following properties:

- If both Higgs bosons $h_{i}$ and $h_{j}$ have non-zero theoretical mass uncertainties $\left(\Delta m_{i} \neq 0\right.$ and $\left.\Delta m_{j} \neq 0\right)$ the combined mass is obtained from a Gaussian average (regardless of the choice for Higgs mass pdf),

$m_{k}=\left(\Delta m_{k}\right)^{2}\left(\frac{m_{i}}{\left(\Delta m_{i}\right)^{2}}+\frac{m_{j}}{\left(\Delta m_{j}\right)^{2}}\right)$,

with the combined theoretical mass uncertainty

$$
\Delta m_{k}=\frac{\Delta m_{i} \Delta m_{j}}{\sqrt{\left(\Delta m_{i}\right)^{2}+\left(\Delta m_{j}\right)^{2}}} .
$$

- If either $m_{i}$ or $m_{j}$ is known exactly, for instance $\Delta m_{i}=0$, the mass of the new Higgs cluster is chosen equal to this mass, $m_{k}=m_{i}$, with zero combined theory mass uncertainty, $\Delta m_{k}=\Delta m_{i}=0$.

- If both $m_{i}$ and $m_{j}$ are known exactly, $\Delta m_{i}=\Delta m_{j}=$ 0 , the Higgs cluster is assigned an averaged mass $m_{k}=\left(m_{i}+m_{j}\right) / 2$, with $\Delta m_{k}=0$.

3 . The procedure is repeated from step 1 . The entities considered for further clustering include both the unclustered (initial) Higgs bosons, as well as the already combined Higgs clusters. The single Higgs bosons which form part of a cluster are no longer present.

4. Each single Higgs boson or Higgs cluster $h_{k}$ that remains after the clustering according to steps 1-3 enters the mass-centered $\chi^{2}$ test. Their predicted signal strength modifiers are formed from the incoherent sum (again, neglecting interference effects) of the individual signal strength modifiers for the combined Higgs bosons,

$$
\mu_{k}\left(m_{k}\right)=\sum_{i} \mu_{i}\left(m_{i}\right)
$$

In this way, the predictions that are compared to one implemented analysis are determined. HiggsSignals repeats this procedure for all implemented experimental analyses.
Since the experimental mass resolution can vary significantly between different analyses, the resulting clustering in each case may differ.

The two different treatments of the theoretical mass uncertainties, as discussed above, have to be slightly extended for the case of Higgs clusters:

(i) If the Higgs boson $h_{i}$ is contained within a Higgs cluster $h_{k}$ for one analysis $a$, the considered mass region for the variation of $m^{\prime}$ in (18) is now the overlap region $M_{i} \cap$ $M_{k}$, with $M_{i}=\left[m_{i}-\Delta m_{i}, m_{i}+\Delta m_{i}\right]$ in the case of a uniform (box) Higgs mass pdf. ${ }^{6}$ We denote the resulting tentative total $\chi^{2}$ from the variation of the mass of Higgs boson $h_{i}$ by $\chi_{i}^{2}$. The variation is done for every Higgs boson contained in the cluster $h_{k}$. When the cluster $h_{k}$ is evaluated against the observed results for analysis $a$, the observed values $\hat{\mu}_{a}$ and $\Delta \hat{\mu}_{a}$ are defined at the value of $m^{\prime}$ where the global $\chi^{2}$, composed of all $\chi_{i}^{2}$ distributions, is minimal. ${ }^{7}$

(ii) In the second approach, the convolution of the experimental $\hat{\mu}$ values with theory uncertainties is performed separately for each Higgs boson, or Higgs cluster $k$, with the combined Higgs mass pdf

$$
g_{k}\left(m^{\prime}, m\right)=\frac{1}{N} \sum_{i} g_{i}\left(m^{\prime}, m\right)
$$

The normalization factor $N=\int_{M_{k}} \mathrm{~d} m^{\prime} g_{k}\left(m^{\prime}, m\right)$ to preserve probability. The sum runs over all Higgs bosons which have been combined for this cluster.

Once all model predictions and mass-centered observables have been defined, when necessary using Stockholm clustering as discussed above, the total mass-centered $\chi^{2}$ is evaluated with a signal strength vector ${ }^{8}$ and covariance matrix constructed analogously as in the peak-centered $\chi^{2}$ method, cf. (6). The uncertainties of production cross sections, decay rates, and the luminosity are again treated as fully correlated Gaussian errors. Note that, in this method, there is no contribution from Higgs mass measurements to the total $\chi^{2}$, since the evaluation is done directly against the experimental data at the predicted Higgs mass values (within their uncertainties).

As a final remark, we would like to point out that the $\hat{\mu}$ plots necessary for this method are so far only published for a

\footnotetext{
${ }^{6}$ If $M_{i} \cap M_{k}=\varnothing$, we increase $M_{k}$ until there is a (minimal) overlap. This will effectively lead to an evaluation of the tentative $\chi^{2}$ at the boundary of $M_{i}$ which is closest to the mass $m_{k}$ of the Higgs cluster.

7 The global $\chi^{2}$ is defined in the mass region $\left(M_{i} \cap M_{k}\right) \cup\left(M_{j} \cap M_{k}\right) \cup$ $\ldots$, when the Higgs bosons $h_{i}, h_{j}, \ldots$ are combined in the cluster $h_{k}$.

8 The length of this vector depends in this case on the Higgs masses and the result of the clustering. Each analysis may contribute any number of entries $\alpha$, where $0 \leq \alpha \leq N_{\text {Higgs }}$.
} 
few selected analyses. ${ }^{9}$ Thus, there is not (yet) a full coverage of the various Higgs signal topologies with the mass-centered $\chi^{2}$ method. Furthermore, the published results cover only a limited range in the Higgs mass, which is a further limit to its applicability.

\subsection{Simultaneous use of both methods}

Since the two methods presented here are complementarythey test inherently different statistical hypothesesHiggsSignals allows for the possibility to apply the peak-centered and mass-centered $\chi^{2}$ methods simultaneously. We present here one approach, which attempts to make maximal use of the available experimental information when testing models with multiple Higgs bosons. The user of HiggsSignals is of course free to use other combinations of the two results, which can be derived completely independently.

In the provided combined approach, HiggsSignals first runs the peak-centered $\chi^{2}$ method and assigns the Higgs bosons to the observed signals, tracing the assigned combination for each analysis. In the second step, all remaining Higgs bosons (which have not been assigned) are considered with the mass-centered $\chi^{2}$ method; their respective (masscentered) $\chi^{2}$ contributions are constructed. In this way, a possible double-counting, where a Higgs boson is tested with both the peak- and mass-centered $\chi^{2}$ method against the same data, is avoided. In the last step, the total $\chi^{2}$ is evaluated. Here, the Higgs mass $\chi^{2}$ from the (relevant) signals, as well as the $\chi^{2}$ from combined signal strength vectors from both the peak-centered and the mass-centered approach, are evaluated with a full covariance matrix. This method thus tests the model predictions against the data in the maximal possible way, while ensuring that no Higgs boson is tested more than once against the same experimental data.

As a final recommendation, it should be noted that the mass ranges for the measured $\hat{\mu}$ values are still much smaller than the mass ranges for (SM) Higgs exclusion limits. To constrain theories with Higgs bosons outside this smaller range (or below the lower limit of the range currently considered by LHC searches), it is still highly recommended to run HiggsBounds [13-15] in parallel to HiggsSignals.

\section{Using HiggsSignals}

\subsection{Installation}

The latest version of HiggsSignals can be downloaded from the webpage http://higgsbounds.hepforge.org which is

\footnotetext{
${ }^{9}$ Currently, the $\hat{\mu}$ plots are published only for the $H \rightarrow \gamma \gamma, H \rightarrow$ $Z Z^{(*)}$ and $H \rightarrow W W^{(*)}$ searches.
}

also the home of HiggsBounds. Since HiggsSignals depends on the HiggsBounds libraries, this code (version 4.0.0 or newer) should be downloaded and installed as well. For further detail on how to do this, we refer to the Higgs Bounds manual [13-15]. Like HiggsBounds, HiggsSignals is written in Fortran 90/2003. Both codes can be compiled, for example, using gfortran (version 4.2 or higher). After unpacking the downloaded source files, which should create a new directory for HiggsSignals, the user possibly needs to set the correct path to the Higgs Bounds installation in the configure file. Optionally, the path to a FeynHiggs installation (version 2.9.4 or higher recommended) [88-92] can be set in order to use some of the example programs which use FeynHiggs subroutines (see below). Furthermore, compiler flags necessary for specific platforms can be placed here. Configuration and installation starts with running

./configure

which will generate a makefile from the initial file makefile.in. Once this is done, run

make

to produce the HiggsSignals Fortran library (called libHS.a) and the command line executable. In addition, the user may conveniently use a bash script,

./run_tests.bat

to build the HiggsSignals library and executable as well as the provided example programs (described in Sect. 4.5). The script will then perform a few test runs.

\subsection{Input and output}

HiggsSignals is designed to require mostly the same input as HiggsBounds, so that users already familiar with this code should be able to transfer their existing analyses to also use HiggsSignals with a minimal amount of extra work. There are two ways to run HiggsSignals: either from the command line, or via the subroutines contained in the HiggsSignals library libHS.a. For the command line version, the model predictions (Higgs masses, their theory uncertainties, total widths, production and decay rates) have to be specified in data files using the same format as HiggsBounds-4, see Ref. [15]. The command line version of HiggsSignals is presented in more detail in Sect. 4.3.

In the subroutine version, the model predictions (which can be given as effective couplings, or as cross sections either at partonic or hadronic level) have to be provided via subroutines. Most of these subroutines are shared with the HiggsBounds library (for details we refer again to [15]). In addition to the HiggsBounds input, HiggsSignals 
Table 1 Example for the SLHA block

HiggsSignalsResults after a successful run of HiggsSignals

The number of observables and $\chi^{2}$ contributions are given separately for the signal strength and mass parts in the peak-centered $\chi^{2}$ method, and also for the mass-centered $\chi^{2}$ method

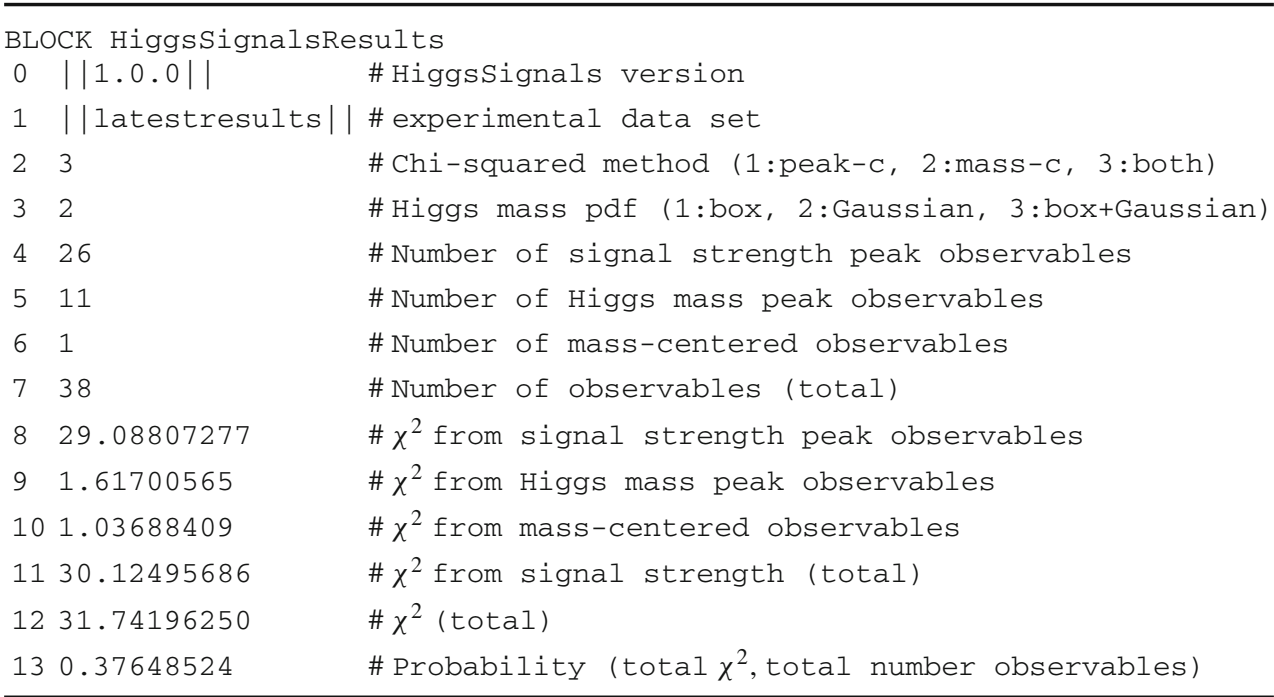

requires input of the theoretical uncertainties on both the Higgs masses and the rate predictions. Therefore, HiggsSignals contains two additional input subroutines to set these quantities, see Sect. 4.4 for more details. An accessible demonstration of how to use the HiggsSignals subroutines is provided by the example programs, discussed further in Sect. 4.5.

As already mentioned, the required input of Higgs production and decay rates can be given either as effective couplings, or as cross sections at partonic or hadronic level. For supersymmetric models there is an option of using the SUSY Les Houches Accord (SLHA) [93,94] for input (either using data files or subroutines). In this case, the production rates are always approximated using the effective couplings specified in the two HiggsBounds specific input SLHA blocks (as specified in Ref. [15]), whereas the Higgs branching ratios are taken directly from the corresponding decay blocks. If present, the theoretical mass uncertainties are read in from the SLHA block DMASS (as available e.g. from FeynHiggs). Otherwise, since there is no consensus yet on how to encode the theoretical rate uncertainties in the SLHA format, these have to be given to Higgs Signals explicitly by hand. ${ }^{10}$

The main results from HiggsSignals are reported in the form of a $\chi^{2}$ value and the number of considered observables. For reference, the code also calculates the $p$ value associated to the total $\chi^{2}$ and the number of degrees of freedom $N$. The user may specify the number of free model parameters $N_{p}$ (see below). Then, the number of degrees

\footnotetext{
$\overline{10}$ This can be done by either calling the subroutine setup_ rate_uncertainties (see below) or by including the rate uncertainties directly in the file usefulbits_HS.f90 in case the subroutine cannot be used (i.e. if HiggsSignals is run on the command line). If the user does not specify the rate uncertainties (in either case), they are assumed to be identical to the SM rate uncertainties, Eq. (9).
}

of freedom is given by $N=N_{\text {obs }}-N_{p}$, where $N_{\text {obs }}$ is the total number of the included observables. Note that if the user does not specify $N_{p}$, the $p$ value is evaluated assuming $N_{p}=0$.

In the case of running with input data files, the HiggsSignals output is written into new files as described in Sect. 4.3. There also exist subroutines, see Sect. 4.4, to specify the extent of screen output and to retrieve many quantities of interest for further analysis.

If HiggsSignals is run in the SLHA mode, the results can be appended to the SLHA file in the form of new SLHAinspired ${ }^{11}$ blocks. The main results are then collected in

\section{BLOCK HiggsSignalsResults,}

as shown for a specific example in Table 1. The first entries of this BLOCK contain general information on the global settings of the HiggsSignals run, i.e. the version number, the experimental data set, the $\chi^{2}$ method and the Higgs mass parametrization used. Moreover, it lists the number of analyzed observables of the different types (BLOCK entries 46 ), as well as the total number (BLOCK entry 7). Next, it gives the corresponding $\chi^{2}$ values separately from the signal strength peak observables (BLOCK entry 8), the Higgs mass peak observables (BLOCK entry 9), and the mass-centered observables (BLOCK entry 10). The total signal strength $\chi^{2}$ for both methods (the sum of BLOCK entries 8 and 10) is provided (BLOCK entry 11 ), as is the total $\chi^{2}$ sum (BLOCK entry 12). The final element (BLOCK entry 13) gives the reference $p$ value, as discussed above.

\footnotetext{
11 These blocks deviate from the SLHA conventions [93,94] in the way that they contain string values (without whitespaces), which are parenthesized by the symbols ' $\mid$ '.
} 
Table 2 Example for the SLHA block HiggsSignals Peak Observables
The first column enumerates through all considered peak observables, as indicated by the dots at the bottom

\begin{tabular}{|c|c|c|c|}
\hline \# OBS & FLAG & VALUE & \# DESCRIPTION \\
\hline 1 & 1 & 201215801 & \#Analysis ID \\
\hline 1 & 2 & ||ATL-CONF-2012-158|| & \# Reference to publication \\
\hline 1 & 3 & || (pp) $->$ h->WW->lnulnu $\mid$ & \# Description (search channel) \\
\hline 1 & 4 & 8.00 & \# Center-of-mass energy ( $\mathrm{TeV})$ \\
\hline 1 & 5 & 13.00 & \# Luminosity $\left(\mathrm{fb}^{-1}\right)$ \\
\hline 1 & 6 & 3.60 & \# Luminosity uncertainty (in \%) \\
\hline 1 & 7 & 8.00 & \# Mass resolution (GeV) \\
\hline 1 & 8 & 126.00 & \# Mass value at peak position (GeV) \\
\hline 1 & 9 & 1.3460 & \# Observed signal strength modifier $(\hat{\mu})$ \\
\hline 1 & 10 & 0.5204 & \# Lower $68 \%$ C.L. uncertainty on $\hat{\mu}$ \\
\hline 1 & 11 & 0.5710 & \# Upper $68 \%$ C.L. uncertainty on $\hat{\mu}$ \\
\hline 1 & 12 & 001 & \# Assigned Higgs combination \\
\hline 1 & 13 & 1 & \# Index of dominant Higgs boson \\
\hline 1 & 14 & 25 & \# PDG number of dominant Higgs boson \\
\hline 1 & 15 & 126.1133 & \# Mass of the dominant Higgs boson \\
\hline 1 & 16 & 0.3305 & \# Signal strength modifier of dom. Higgs \\
\hline 1 & 17 & 0.3305 & $\begin{array}{l}\text { \# Total predicted signal strength } \\
\text { modifier } \mu\end{array}$ \\
\hline 1 & 18 & 1.6196 & $\# \chi^{2}$ from signal strength \\
\hline 1 & 19 & 0.0000 & $\# \chi^{2}$ from Higgs mass \\
\hline 1 & 20 & 1.6196 & $\# \chi^{2}($ total $)$ \\
\hline 1 & 21 & 2.3514 & $\# \chi^{2}$ for no predicted signal $(\mu=0)$ \\
\hline 2 & 1 & 201209202 & \# Analysis ID \\
\hline 2 & 2 & ||ATL-CONF-2012-092|| & \# Reference to publication \\
\hline 2 & 3 & ||$(p p)->h+\ldots->z Z->41||$ & \# Description (search channel) \\
\hline$\vdots$ & $\vdots$ & $\vdots$ & $\vdots$ \\
\hline
\end{tabular}

Additional output specific to the peak-centered $\chi^{2}$ method is collected in

\section{BLOCK HiggsSignals PeakObservables.}

We show an excerpt from this extensive BLOCK for an example (MSSM) parameter point in Table 2. The first identifier, OBS, in the BLOCK enumerates the peak observables, whereas the second number, FLAG, labels the specific quantity (for this peak observable). For every peak observable, the first entries $(F L A G=1-11)$ give general information about the experimental data defining the observable. This is followed by model-specific information and the results from the Higgs Signals run. FLAG $=12$ displays a binary code representing the Higgs boson combination which has been assigned to the signal. It has the same length as the number of Higgs bosons, ${ }^{12}$ such that an assigned Higgs boson with index $k$ corresponds to the binary value $2^{k-1}$. A code of only

\footnotetext{
12 For technical reasons, Higgs Signals is currently limited to models with $n_{H} \leq 9$ neutral Higgs bosons, but this could easily be extended if there is a demand for more.
}

zeroes means that no Higgs boson has been assigned to this peak observable. In the specific example shown in Table 2, the lightest of the three neutral Higgs bosons in the MSSM (with $k=1$ ) has been assigned.

This BLOCK also contains additional information (index $i$, Particle data group (PDG) number, mass, and signal strength contribution under FLAG $=13-16$ ) about the assigned Higgs boson that gives the largest contribution to the total predicted signal strength. The total predicted signal strength is given by FLAG=17. The HiggsSignals results (FLAG=18-20) contain the $\chi^{2}$ contribution from the signal strength and Higgs mass test from this observable, as well as the total $\chi^{2}$ contribution obtained for the assigned Higgs boson combination. Finally, the $\chi^{2}$ obtained for the case with no predicted signal, $\mu=0$, is given for FLAG $=21$. It should be noted that the quoted $\chi^{2}$ values correspond to intermediate results in the total $\chi^{2}$ evaluation, where correlated uncertainties are taken into account by the covariance matrix. For instance, the signal strength $\chi^{2}(\mathrm{FLAG}=18)$ corresponds to $\chi_{\mu, \alpha}^{2}$ in Eq. (6), where $\alpha$ is the index of the peak observable given in the first column of the BLOCK. Thus, this quantity differs from the 
Table 3 Example for the SLHA output block

HiggsSignalsMass

Centeredobservables

containing information about the observables and results from the mass-centered $\chi^{2}$ method

\begin{tabular}{|c|c|c|c|}
\hline \# OBS & FLAG & VALUE & \# DESCRIPTION \\
\hline 1 & 1 & 201215801 & \# Analysis ID \\
\hline 1 & 2 & ||ATL-CONF-2012-158|| & \# Reference to publication \\
\hline 1 & 3 & ||$(p p)->h->W W->\operatorname{lnulnu}||$ & \# Description (search channel) \\
\hline 1 & 4 & 8.00 & \# Center-of-mass energy (TeV) \\
\hline 1 & 5 & 13.00 & \# Luminosity $\left(f b^{-1}\right)$ \\
\hline 1 & 6 & 3.60 & \# Luminosity uncertainty (in) \\
\hline 1 & 7 & 8.00 & \# Mass resolution (GeV) \\
\hline 1 & 8 & 122.65 & $\begin{array}{l}\text { \# Mass of tested Higgs } \\
\text { boson (GeV) }\end{array}$ \\
\hline 1 & 9 & 2.00 & $\begin{array}{l}\text { \# Mass uncertainty of } \\
\text { tested Higgs boson (GeV) }\end{array}$ \\
\hline 1 & 10 & 0.7379 & $\begin{array}{l}\text { \# Signal strength of } \\
\text { tested Higgs boson(s) }\end{array}$ \\
\hline 1 & 11 & 1 & \# Number of combined Higgs bosons \\
\hline 1 & 12 & 001 & \# Combined Higgs boson code \\
\hline 1 & 13 & 122.90 & \# Observed mass value (GeV) \\
\hline 1 & 14 & 1.8269 & \# Observed signal strength $\hat{\mu}$ \\
\hline 1 & 15 & 0.6822 & \# Lower $68 \% \mathrm{C} . \mathrm{L}$. uncertainty on $\hat{\mu}$ \\
\hline 1 & 16 & 0.7462 & \# Upper $68 \%$ C.L. uncertainty on $\hat{\mu}$ \\
\hline 1 & 17 & 2.9617 & $\# \chi^{2}($ total $)$ \\
\hline 2 & 1 & 201209202 & \# Analysis ID \\
\hline 2 & 2 & ||ATL-CONF-2012-092|| & \# Reference to publication \\
\hline 2 & 3 & ||$(p p)->h+\ldots->z Z->41||$ & \# Description (search channel) \\
\hline$\vdots$ & $\vdots$ & $\vdots$ & $\vdots$ \\
\hline
\end{tabular}

naïvely calculated $\chi^{2}=(\mu-\hat{\mu})^{2} /(\Delta \hat{\mu})^{2}$, and might in the extreme case even be negative due to the impact of correlated uncertainties.

The results from the mass-centered $\chi^{2}$ method are summarized in

\section{BLOCK HiggsSignalsMassCenteredobservables}

in a similar way as in BLOCK HiggsSignalsPeak Observables. An example is given in Table 3. The modelindependent information about the observable $(F L A G=1-7)$ is identical to the corresponding information in BLOCK HiggsSignals PeakObservables. However, since the evaluated experimental quantities of the mass-centered observable depend on the model prediction, cf. Sect. 3.2, we give the information of the tested Higgs boson (cluster) at first ( $\mathrm{FLAG}=8-10)$, corresponding to Eqs. (22)(24). The number and binary code of the combined Higgs bosons, which form a Stockholm Higgs cluster, is given by $F L A G=11$ and 12 , respectively. From the experimental data is given the mass position ( $F L A G=13)$, and the measured signal strength with its lower and upper uncertainties $(F L A G=14-16)$. Finally, the resulting $\chi^{2}$ con- tribution from this mass-centered observable is given at $F L A G=17$.

Note that there is also the possibility to create a new SLHA file with the HiggsSignals output blocks even if the input was not provided in SLHA format. Moreover, HiggsSignals can give an extensive screen output with similar information as encoded in the three SLHA output blocks. The level of information that is desired should then be specified before the HiggsSignals run via the subroutine setup_output_level. See Sect. 4.4 for more details.

\subsection{Running HiggsSignals on the command line}

Higgs Signals can be run on the command line as follows:

$$
\begin{aligned}
& \text {./HiggsSignals }<\text { expdata }><\text { mode }><\text { pdf }> \\
& <\text { whichinput }><\text { nHzero }><\text { nHplus }><\text { prefix }>
\end{aligned}
$$

This command line call is very similar to the one of Higgs Bounds and the last four arguments have been directly taken over from HiggsBounds. The user may consult the HiggsBounds manual [15] for more details on these arguments. The number of neutral and charged Higgs bosons of 
the model are specified by nHzero and nHplus, respectively. As in HiggsBounds, the model predictions are read in from the data files specified by prefix. Which data files are required as input depends on the argument whichinput, which can take the string values effC, part, hadr and SLHA for the various input formats. The theory mass uncertainties are read in from the data file $<$ prefix>MHall_uncertainties.dat for both the neutral and charged Higgs bosons. If this file is absent these uncertainties are set to zero. For more information of the data file structure we refer to the HiggsBounds-4 manual [15]. Note that for whichinput=SLHA, all the input is read in from the SLHA input file which, like the ordinary data files, should be specified by $<$ prefix $>$.

The first three arguments are intrinsic HiggsSignals options. The string < expdata $>$ specifies which experimental data set should be used. HiggsSignals will read in the observables found in the directory Expt_tables / $<$ expdata $>$. The second argument, $<$ mode $>$, specifies which $\chi^{2}$ method should be used; it can take the string values peak (for the peak-centered $\chi^{2}$ method, described in Sect. 3.1), mass (for the mass-centered $\chi^{2}$ method, see Sect. 3.2), or both (for the simultaneous use of both methods, as described in Sect. 3.3). Finally, the $<p d f>$ argument takes an integer selecting the parametrization for the Higgs mass uncertainty as either 1 (box), 2 (Gaussian), or 3 (box+Gaussian) pdf.

As an example, the user may run

./HiggsSignals latestresults peak 2 effC 31 example_data/mhmax/mhmax_

which runs the peak-centered $\chi^{2}$ method on the provided parameter points in the $\left(M_{A}, \tan \beta\right)$ plane of the $m_{h}^{\max }$ benchmark scenario [95] of the MSSM, using the most recent Higgs data contained in the directory Expt_tables / latestresults/.

The HiggsSignals output from a successful command line run is collected in the data file <prefix $>$ HiggsSignals_results.dat, except for the case whichinput=SLHA, where the results are attached as SLHA output blocks to the SLHA file, cf. Sect. 4.2. The SUSY spectrum generator SPheno $[96,97]$, used in conjunction with the model building tool SARAH [98-100], can write directly the HiggsBounds (and thus HiggsSignals) data files for input in the effective couplings format.

\subsection{HiggsSignals subroutines}

In this section we present the subroutines needed for the use of HiggsSignals. First, we go step-by-step through the user subroutines encountered during a normal run of
HiggsSignals. Then, we list additional (optional) subroutines for specific applications of HiggsSignals, and for a convenient handling of the output.

\section{Main user subroutines}

The subroutine that is usually called first is

initialize_HiggsSignals(int nHzero, int nHplus, char* expdata)

which sets up the HiggsSignals framework: It allocates internal arrays according to the number of neutral (nHzero) and charged ${ }^{13}$ (nHplus) Higgs bosons in the model and reads in the tables for the SM branching ratios in the same way as done in HiggsBounds. Furthermore, it calls the subroutine setup_observables, which reads in the experimental data contained in the directory Expt_tables/(expdata). The user may create a new directory in Expt_tables/ containing the relevant observables for his study, see Sect. 4.6 for more details. For convenience, we also provide a wrapper subroutine

initialize_HiggsSignals_latestresults(int nHzero, int nHplus)

which does not require the third argument but uses the experimental data from the folder Expt_tables/ latestresults/.

\section{setup_pdf(int pdf)}

The next step is to specify the probability density function (pdf) for the Higgs masses, which is done using setup_pdf. Available settings are pdf $=1$ for a uniform (box-shaped) distribution, $\mathrm{pdf}=2$ for a Gaussian, and $\mathrm{pdf}=3$ for a box-shaped pdf with Gaussian tails. The impact of this choice has been discussed in detail in Sect. 3 and will furthermore be demonstrated in Sect. 5. With the subroutine

HiggsSignals_neutral_input_MassUncertainty(double(nHzero) dMh)

values for the theory mass uncertainties $\Delta m_{i}$ can be specified. This subroutine sets the theoretical uncertainties of the neutral Higgs boson masses (in $\mathrm{GeV}$ ) of the model via the array dMh. The default values (in case this subroutine is not invoked) is for all uncertainties to be zero. Note that HiggsBounds-4 also contains a similar subroutine (set_mass_uncertainties) to set theoretical

\footnotetext{
$\overline{13}$ At this point, there are no measurements available of signal strength quantities for charged Higgs bosons, which are therefore not considered in any way by Higgs Signals.
} 
Table 4 Ordering of the elements of the input arrays $\mathrm{ACS}$ and $\mathrm{ABR}$ for the relative uncertainties of the hadronic production cross sections and branching ratios, respectively

\begin{tabular}{|c|c|c|c|c|c|}
\hline \multirow[t]{2}{*}{ Array } & \multicolumn{5}{|l|}{ Element } \\
\hline & 1 & 2 & 3 & 4 & 5 \\
\hline $\mathrm{dCS}$ & singleH & VBF & $H W$ & $H Z$ & $t \bar{t} H$ \\
\hline $\mathrm{dBR}$ & $H \rightarrow \gamma \gamma$ & $H \rightarrow W W$ & $H \rightarrow Z Z$ & $H \rightarrow \tau \tau$ & $H \rightarrow b \bar{b}$ \\
\hline
\end{tabular}

Recall that the hadronic production mode "singleH" usually contains both the partonic processes $g g \rightarrow H$ and $b \bar{b} \rightarrow H$, currently assuming equal experimental efficiencies. The latter can change in the future once search categories with $b$-tags are included. This table will possibly be extended once measurements in new channels (e.g. $H \rightarrow Z \gamma$ ) are performed

mass uncertainties of the neutral and charged Higgs bosons. These uncertainties are taken into account via mass variation in the HiggsBounds run. Since the treatment of these uncertainties is intrinsically different between the two codes, we allow the user to set the theoretical mass uncertainties for HiggsSignals independently using this subroutine. ${ }^{14}$

setup_rate_uncertainties(double(5) dCS, double(5) dBR)

For models with different uncertainties on the Higgs production cross sections and branching ratios than those for a SM Higgs boson, these should be specified using this subroutine, which sets the theoretical uncertainties of the production and decay rates (in \%) in the considered model. In the current implementation, LHC and Tevatron channels are considered to have the same relative rate uncertainties, and the rate uncertainties are assumed to be the same for all neutral Higgs bosons, independent of their masses. The input arrays should follow the structure of Table 4.

The remaining required input (Higgs boson masses, total widths, branching ratios, cross sections) is identical to the HiggsBounds input and should be set via the HiggsBounds input subroutines, cf. Ref. [15].

\section{setup_nparam (int $\mathrm{Np}$ )}

In order to evaluate a meaningful $p$ value during the HiggsSignals run, the program has to know the number of free model parameters, $N_{p}$, cf. Sect. 4.2. This number is specified by the subroutine setup_nparam. If this subroutine is not called before the main HiggsSignals run, the code assumes no free model parameters, $N_{p}=0$.

run_HiggsSignals (int mode, double csqmu, double csqmh, double csqtot, int nobs, double Pvalue)

\footnotetext{
$\overline{14}$ The use of different theoretical mass uncertainties in HiggsBounds and HiggsSignals is restricted to the subroutine version. In the command line version of both programs, the theoretical uncertainties will be read in from the same data file, namely $<$ prefix >MHall_uncertainties.dat.
}

Once all the input has been specified, the main HiggsSignals evaluation can be run by calling the run _HiggsSignals subroutine to start the $\chi^{2}$ evaluation. The mode flag specifies the $\chi^{2}$ method which is used in the following evaluation process. Possible values are mode $=1$ (peak-centered method, cf. Sect. 3.1), mode $=2$ (masscentered method, cf. Sect. 3.2), or mode $=3$ (simultaneous use of both methods, cf. Sect. 3.3). After a successful run, this subroutine returns the $\chi^{2}$ contribution from the signal strength measurements (csqmu), ${ }^{15}$ the $\chi^{2}$ contribution from the Higgs mass measurements (csqmh), and the total $\chi^{2}$ value (csqtot). It also returns the number of observables involved in the $\chi^{2}$ evaluation (nobs). If the mass-centered $\chi^{2}$ method is employed, it is important to realize that nobs can depend on many parameters, such as the Higgs boson masses of the model (which may be inside or outside the range of an analysis). The Stockholm clustering can also affect the number of observables that are evaluated in the final $\chi^{2}$ calculation. Finally, the associated $p$ value (Pvalue) for the total $\chi^{2}$ with nobs $-N_{p}$ degrees of freedom is calculated.

$$
\text { finish_HiggsSignals () }
$$

At the end of a HiggsSignals run, the user should call this routine to deallocate all internal arrays.

\section{Specific user subroutines}

This section provides a list (alphabetically ordered) of subroutines handling more special features of Higgs Signals.

assign_toyvalues_to_peak(int obsID, double mu_obs, double mh_obs)

If the user wants to perform a dedicated statistical study using pseudo-measurements (also called toy-measurements) for the Higgs signal rates and mass measurements, they can be set via this subroutine for the peak observable with the identification number obsID. This observable ID is unique

\footnotetext{
15 If mode $=3$, csqmu contains the contributions from peak and mass-centered observables.
} 
to the peak observable and is encoded in the experimental data, see Sect. 4.6 for more details. After a (dummy) run of HiggsSignals the observable ID can also be read out with the subroutine get_ID_of_peakobservable (see below). The arguments mu_obs and mh_obs are the pseudo-measured values for the signal strength modifier $\hat{\mu}$ and the Higgs mass $\hat{m}$. Note that the uncertainties are kept at their original values.

assign_rate_uncertainty_scalefactor_to_peak (int obsID, double scale_mu)

If the user wants to scale the uncertainties of the Higgs signal rate and mass measurements, this can be done via this subroutine in an analogous way as setting the toy measurements (using assign_toyvalues_to_peak). Here, scale_mu is the scale factor for the experimental uncertainty on the signal strength of the peak with identification number obsID. The theoretical rate uncertainties, which can be set independently via the subroutine setup_rate_uncertainties (see above), are unaffected by this scale factor. In this way, HiggsSignals allows the user to scale the experimental and theoretical rate uncertainties independently. This is useful if the user is interested in a future projection of the compatibility between the model and the experimental data, assuming that a certain improvement in the precision of the measurements and/or theoretical predictions can be achieved.

After the HiggsSignals run the user can employ the following "get_" subroutines to obtain useful information from the HiggsSignals output. The following three subroutines are contained in the Fortran module io.

get_ID_of_peakobservable(int i, int obsID)

If the peak-centered $\chi^{2}$ method is used, the peak observables are internally enumerated in HiggsSignals based on their alphabetical appearance in the directory Expt_ tables/ (expdata) of the used experimental dataset. This ordering is reflected e.g. in the screen output and the SLHA output. However, a safer way to access the peak observables (for instance to set toy observables) is to use the unique observable ID of the peak observable. For this, the user may call this subroutine which returns the observable ID obs ID internally structured at the position $i$.

get_number_of_observables(int ntotal, int npeakmu, int npeakmh, int nmpred, int nanalyses)

This subroutine returns the total number of various observables: ntotal is the total number of observables, npeakmu and npeakmh are the number of signal strength and Higgs mass observables entering the peak-centered $\chi^{2}$ method, respectively, nmpred is the number of observables consid-
Table 5 Channels codes used for Higgs production and decay modes, for example by the get_rates subroutine (see text for details)

\begin{tabular}{llll}
\hline 1st digit & Production mode & 2nd digit & Decay mode \\
\hline 1 & singleH & 1 & $H \rightarrow \gamma \gamma$ \\
2 & VBF & 2 & $H \rightarrow W W$ \\
3 & $H W$ & 3 & $H \rightarrow Z Z$ \\
4 & $H Z$ & 4 & $H \rightarrow \tau \tau$ \\
5 & $t \bar{t} H$ & 5 & $H \rightarrow b \bar{b}$ \\
\hline
\end{tabular}

ered in the mass-centered $\chi^{2}$ method, and nanalyses gives the number of implemented analyses. Note that several masscentered and peak observables can in general exist for each experimental analysis.

get_peakinfo_from_HSresults(int obsID, double(npeak) mupred, int (npeak) domH, int(npeak) nHcomb)

More information about the HiggsSignals result can be obtained by calling this subroutine. It returns the total predicted signal strength modifier, the index of the dominantly contributing Higgs boson and the number of combined Higgs bosons for the peak observable with observable identifier obsID as mupred, npeak and nHcomb, respectively.

\section{get_Pvalue(int $\mathrm{Np}$, double Pvalue)}

The user may apply the subroutine get_Pvalue to evaluate the $p$ value again after run_HiggsSignals, with the possibility to vary $N_{p}$. The result is based on the total $\chi^{2}$ and the total number of observables from the last HiggsSignals run as well as the number of free parameters, Np, which are passed as input to this subroutine.

get_rates(int i, int collider, int Nchannels, int(Nchannels) IDchannels, double rate)

This subroutine allows the user to read out the predicted signal rate for an arbitrary channel combination. This channel combination is specified by the number of combined channels, Nchannels, and the array IDchannels, which contains the two-digit IDs of these channels as specified in cf. Table 5. The output (rate) is the combined rate. It is more general than get_Rvalues (see below).

get_Rvalues(int i, int collider, double R_H_WW, double R_H_ZZ, double R_H_gaga, double R_H_tautau, double R_H_bb, double R_VH_bb)

This returns the model-predicted signal rates (normalized to the SM signal rates) of Higgs boson i for the six different processes listed in Table 6 . These signal rates are calculated via Eq. (1), assuming that all channels have the same relative 
Table 6 Production and decay modes considered in the signal rate ratio quantities which are returned by the subroutine get_Rvalues

\begin{tabular}{lll}
\hline Argument & Production modes & Decay mode \\
\hline R_H_WW & singleH, VBF, $H W, H Z, t \bar{t} H$ & $H \rightarrow W W$ \\
R_H_ZZ & singleH, VBF, $H W, H Z, t \bar{t} H$ & $H \rightarrow Z Z$ \\
R_H_gaga & singleH, VBF, $H W, H Z, t \bar{t} H$ & $H \rightarrow \gamma \gamma$ \\
R_H_tautau & singleH, VBF, $H W, H Z, t \bar{t} H$ & $H \rightarrow \tau \tau$ \\
R_H_bb & singleH, VBF, $H W, H Z, t \bar{t} H$ & $H \rightarrow b \bar{b}$ \\
R_VH_bb & $H W, H Z$ & $H \rightarrow b \bar{b}$ \\
\hline
\end{tabular}

efficiency, $\epsilon_{i}=1$. These quantities are evaluated either for the Tevatron or LHC with $\sqrt{s}=7 \mathrm{TeV}$ or $8 \mathrm{TeV}$, as specified by the argument collider, taking the values 1, 2 or 3 for Tevatron, LHC7 or LHC8, respectively.

In order to write the HiggsSignals SLHA output blocks, we provide three different SLHA output subroutines, contained in the Fortran module io. For more information about these output blocks, see Sect. 4.2.

HiggsSignals_create_SLHA_output(char* filename, int detailed)

If the user does not use the SLHA input format of Higgs Signals, or rather wants to write the output into a different file, this subroutine can be used to create a new file as specified by the argument filename. If this file already exists, HiggsSignals will not overwrite this file but give a warning. The integer argument detailed takes values of 0 or 1 , determining whether only the block HiggsSignalsResults or all possible output blocks (i.e. also the block HiggsSignals PeakObservables and/or Higgs SignalsMassCenteredobservables), respectively, are written to the file. The wrapper subroutine

HiggsSignals_create_SLHA_output_default( int detailed)

does the same but for the default filename called HSoutput.slha.

HiggsSignals_SLHA_output(int detailed)

If HiggsSignals is run on an SLHA input file, the subroutine HiggsSignals_SLHA_output appends the HiggsSignals results as blocks to the SLHA input file.

The following "setup_" subroutines can be used to change the default settings of the HiggsSignals run. Thus, they should be called before the subroutine run_ Higgssignals.

setup_assignmentrange(double Lambda)
This subroutine can be used to change the mass range, in which a Higgs boson is forced to be assigned to a peak observable, see Sect. 3.1.3. The value Lambda corresponds to $\Lambda$ in Eq. (15).

setup_correlations(int corr_mu, int corr_mh)

The subroutine can be used to switch off (on) the correlations among the systematic uncertainties in the $\chi^{2}$ evaluation of the signal strength [Higgs mass] part by setting corr_mu [Corr_mh] $=0(1)$. If this subroutine is not called, the default is to evaluate the $\chi^{2}$ with correlated uncertainties (corr_mu = corr_mh $=1)$.

setup_mcmethod_dm_theory ( int mode)

If the mass-centered $\chi^{2}$ method is used, the treatment of the Higgs mass theory uncertainty can be set by calling this subroutine with mode $=1$ to use the mass variation (default), or mode $=2$ for convolving the theory mass uncertainty with the $\hat{\mu}$ plot. See Sect. 3.2 for more details of these methods.

setup_output_level(int level)

The user may control the screen output from the HiggsSignals run with the subroutine, where level takes values from 0 to 3 , corresponding to the following output:

0 Silent mode (suitable for model parameter scans, etc.) (default),

1 Screen output for each analysis with its peak and/or masscentered observables. The channel signal strength modifiers and SM channel weights, cf. Eqs. (2) and (3), respectively, are given for all channels considered by the analysis.

2 Screen output of the essential experimental data of the peak observables and/or implemented $\hat{\mu}$ plots (as used for the mass-centered $\chi^{2}$ method). For each observable, the signal channels are listed with the implemented efficiencies.

3 Creates text files holding essential information about the experimental data and the model predictions for each observable. In the peak-centered $\chi^{2}$ run mode, the files peak_information.txt and peak_ massesandrates. txt are created. The first file lists all peak observables, including a description and references to the publications, whereas the second file gives the observed and model-predicted values for the Higgs mass ${ }^{16}$ and signal rates and their corresponding pull

\footnotetext{
16 If multiple Higgs bosons are assigned to the peak, we give the mass of the Higgs boson contributing dominantly to the signal rate.
} 
values, which we define as:

pull value $=\frac{\text { predicted value }- \text { observed value }}{\text { (Gaussian combined }) \text { uncertainty }}$

Note that in this expression the effect of correlated uncertainties is not taken into account. In the mass-centered $\chi^{2}$ run mode, the files mctables_information.txt and mcobservables_information.txt are created. The first file gives general information about the analyses with an implemented $\hat{\mu}$-plot. The second file lists all mass-centered observables, which have been constructed during the HiggsSignals run, including the mass position, the observed and predicted signal strength values as well as their pull values.

For any of the options level $=1-3$, the main HiggsSignals results are printed to the screen at the end of the run.

\subsection{Example programs}

HiggsSignals provides the seven example programs HSeffC, HShadr, HSwi thSLHA, HBandHSwi thSLHA, HSwithToys, HS_scale_uncertainties, and HBandHSwithFH. They are contained in the subfolder

./example_programs /

of the main HiggsSignals distribution and can be compiled all together (except HBandHSwi thFH) by running

make HSexamples

or separately by calling:

make < name of example program $>$

The first program, HSeffC, considers a model with one neutral Higgs boson and uses the effective couplings input subroutines of HiggsBounds to set the input. It demonstrates how to scan over a certain Higgs mass range and/or over various effective couplings while calculating the total $\chi^{2}$ for every scan point. The code furthermore contains two functions: get_g2hgaga, which calculates the loopinduced $H \gamma \gamma$ effective coupling from the effective (treelevel) Higgs couplings to third generation fermions and gauge bosons [101] (assuming a Higgs boson mass of $126 \mathrm{GeV}$ ), and a second function which interpolates the cross section uncertainty of the composed single Higgs production from the uncertainties of the gluon fusion and $b \bar{b} \rightarrow H$ processes using the effective $H g g$ and $H b \bar{b}$ couplings. This can be relevant if the Higgs coupling to bottom quark is strongly enhanced.
The second example program, HShadr, performs a two dimensional scan over common scale factors of the hadronic production cross sections of $p \stackrel{(-)}{p} \rightarrow H$ and $p \stackrel{(-)}{p} \rightarrow t \bar{t} H$ on the one side, denoted by $\mu_{g g f+t t H}$, and of $p \stackrel{(-)}{p} \rightarrow q \bar{q} H$, $p \stackrel{(-)}{p} \rightarrow W H$ and $p \stackrel{(-)}{p} \rightarrow Z H$ on the other side, denoted by $\mu_{\mathrm{VBF}+V H}$. The Higgs branching ratios are kept at their SM values.

The third example program, HSwithSLHA, uses the SLHA input of HiggsBounds, i.e. an SLHA file which contains the two special input blocks for HiggsBounds. It can be executed with

\section{./HSwithSLHA < number of SLHA files > $<$ SLHAfilename $>$}

The program can test several SLHA files in one call. The total number of SLHA files must therefore be given as the first argument. The SLHA files must all have the same name, and should be enumerated by SLHA_filename.x, where $\mathrm{x}$ is a number. Running, for example,

\section{./HSwithSLHA 2 SLHAexample.fh}

would require the two SLHA files SLHAexample. fh. 1 and SLHAexample. fh. 2 to be present. The output is written as SLHA blocks, cf. Sect. 4.2, which are appended to each input SLHA file. The example program HBandHSwi thSLHA can be run in an analogous way. It employs both HiggsBounds and HiggsSignals on the provided SLHA file(s), demonstrating how these two codes can be run together efficiently.

The example program HSwi thToys demonstrates how to set new values (corresponding to pseudo-measurements) for $\hat{\mu}$ and $\hat{m}$ for each signal. In the code, HiggsSignals is first run on the SM with a Higgs mass around $126 \mathrm{GeV}$ using the effective couplings input format. Then, the predicted signal strengths are read out from the HiggsSignals output and set as pseudo-measurements. A second HiggsSignals run on these modified observables then results in a total $\chi^{2}$ of zero.

The example program HS_scale_uncertainties also runs on the SM with a Higgs mass around $126 \mathrm{GeV}$. It scans over a universal scale factor for $(i)$ the experimental uncertainty of the signal strength $\hat{\mu}$ only, (ii) the theoretical uncertainties of the production cross sections and branching ratios only, and finally (iii) both experimental and theoretical uncertainties. The output of each scan is saved in text files. In this way, rough projections of the model compatibility to a more accurate measurement in the future (with the same central values) can be made.

The last example, HBandHSwi thFH, demonstrates how to run HiggsBounds and HiggsSignals simultaneously on a realistic model, in this case the MSSM. Here, FeynHiggs [88-91] is used to calculated the MSSM pre- 
Table 7 Example file for an implemented peak observable

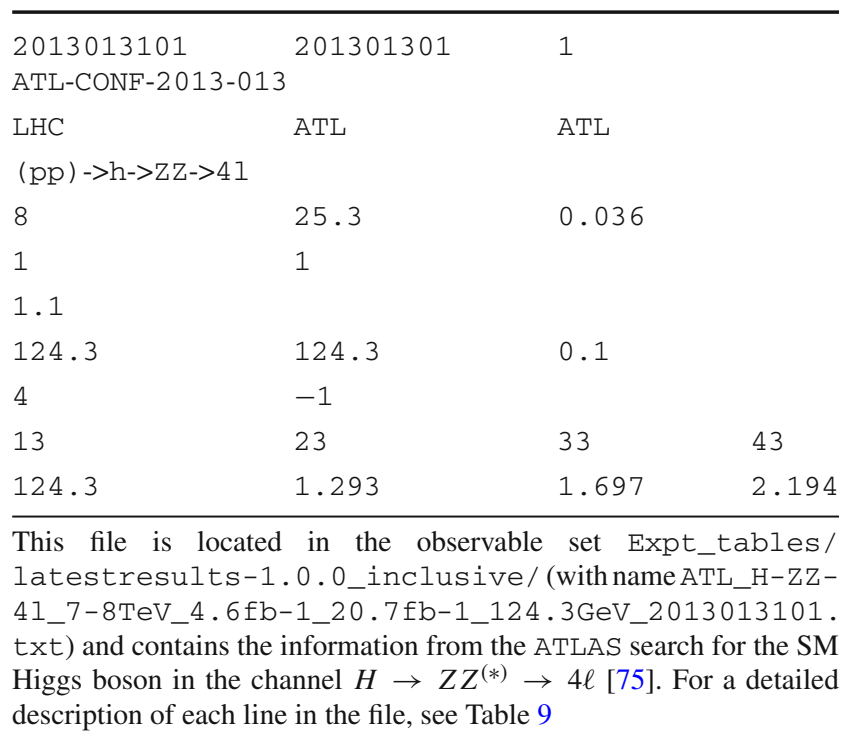

dictions needed as input for HiggsBounds and Higgs Signals.

\subsection{Input of new experimental data into HiggsSignals}

The ambition with HiggsSignals is to always keep the code updated with the latest experimental results. Nevertheless, there are several situations when a user may want to manually add new data (or pseudo-data) to the program, for example to assess the impact of a hypothetical future measurement. For advanced users, we therefore provide a full description of the data file format used by HiggsSignals.

For each observable that should be considered by Higgs Signals, there must exist a textfile (file suffix: . txt). This file should be placed in a directory

\section{Expt_tables/(expdata)/}

where (expdata) is the name identifying the new (or existing) experimental dataset. ${ }^{17}$ All analysis files in this directory will then be read in automatically by HiggsSignals during the initialization.

As an example we show in Tables 7 and 8 the two data files for the inclusive measurement of the ATLAS $H \rightarrow$ $Z Z^{(*)} \rightarrow 4 \ell$ analysis [75], which define a peak observable and provide the full $\hat{\mu}$ plot as needed by the mass-centered $\chi^{2}$ method, respectively. The first 11 rows of these files encode general information about the analysis and the observable (each row is required), as described in Table 9. Comments can be included in the top rows if they are starting with a \# symbol. Note that the observable ID must be unique,

\footnotetext{
17 The identifier (expdata) is the argument which has to be passed to initialize_HiggsSignals at initialization, cf. Sect. 4.4.
}

Table 8 Example file for an analysis with a full $\hat{\mu}$ plot as needed for the mass-centered $\chi^{2}$ method

\begin{tabular}{llll}
\hline 2013013201 & 201301301 & 2 & \\
ATL-CONF-2013-013 & ATL & ATL & \\
LHC & & & \\
$(\mathrm{pp})->$ h->ZZ->41 & & & \\
8 & 25.3 & 0.036 & \\
1 & 1 & & \\
1.1 & & & \\
110.0 & 180.0 & 0.1 & \\
4 & -1 & 33 & -0.1845 \\
13 & 23 & -0.6395 & -0.1730 \\
110.0 & -0.6568 & -0.6384 & -0.1615 \\
110.1 & -0.6563 & -0.6372 & -0.1499 \\
110.2 & -0.6558 & -0.6361 & -0.1384 \\
110.3 & -0.6552 & -0.6349 & $\vdots$ \\
110.4 & -0.6547 & $\vdots$ & \\
$\vdots$ & $\vdots$ & & \\
\hline
\end{tabular}

This file is located in Expt_tables/latestresults-1.0.0_ inclusive/ (with name ATL_H-ZZ-41_7-8TeV_4.6fb-1_ $\left.20.7 \mathrm{fb}-1 \_2013013201 . \mathrm{txt}\right)$. It is the same analysis for which we already defined a peak observable in Table 7. For a detailed description of each line in the file, see Table 9

whereas the analysis ID must be the same for (peak- or masscentered) observables, which correspond to the same analysis and where a multiple assignment of the same Higgs boson to the corresponding observables shall be avoided. In the (yet hypothetical) case that two distinct signals have been observed within the same analysis, their peak observables thus need to have the same analysis ID, otherwise a Higgs boson might be assigned to both signals. All integers should not have more than 10 digits.

The channel codes in the 10th row are given as two-digit integers, where the first digit encodes the production mode, and the second digit the decay mode. The corresponding numbers are given in Table 5. For example, the channel code of $(p p) \rightarrow H W \rightarrow(b \bar{b}) W$ is 35. In the example of Table 7, we thus consider all five production modes, but only a single decay mode, i.e. $H \rightarrow \gamma \gamma$.

Channel efficiencies can be included in the 11th row. They correspond to the channels as defined by the channel codes on the previous row, and thus have to be given in the same order. If the experimental channel efficiencies are unknown (as in the given example of an inclusive measurement), the reference mass in the 9th row should be set equal to -1 , in which case the 11th row will be ignored. Since it must still be present, it could be left blank for the sake of clarity. Note that the channel efficiencies are defined as the fraction of events passing the analysis cuts, and not the relative contribution of this channel to total signal yield. The latter would 
Table 9 Input format for general analysis information encoded in the first 11 rows of the experimental data file

\begin{tabular}{ll}
\hline Row & Description \\
\hline 1 & Observable ID, Analysis ID, Observable type (1: peak, 2: mass) \\
2 & Publication reference \\
3 & Collider ID, Collaboration ID, Experiment ID \\
4 & Description of the search channel \\
5 & CM energy (TeV), Integrated luminosity $\left(\mathrm{fb}^{-1}\right)$, Relative luminosity uncertainty \\
6 & Higgs boson type (1: neutral, $2:$ charged), Enable $\chi^{2}$ from $m_{H}$ (0: no, 1: yes) \\
7 & Mass resolution of analysis (GeV), assignment group (optional string without whitespaces) \\
8 & Lowest Higgs mass, highest Higgs mass, Higgs mass interval (of the following datatable) \\
9 & Number of search channels, reference mass for efficiencies (-1: no efficiencies given) \\
10 & Search channel codes (see Table 5) (\# entries must equal \# channels)) \\
11 & Channel efficiencies (\# entries must equal \# channels) \\
\hline
\end{tabular}

use information about the channel cross section, which in our case is already taken care of by the channel weights $\omega$, cf. Eq. (3). Furthermore, it is only the relative efficiencies among the channels that are important, and not their overall normalization (for the same reason). We therefore typically normalize the relative efficiencies such that the first element in the 11th row is equal to 1 . As an example, the user may investigate one of the category measurements provided in the folder Expt_tables/latestresults-1.0.0/.

From the 12th row onwards, the signal strength data is listed. Each row contains four values: the Higgs mass, the measured signal strength modifier at the lower edge of the $1 \sigma$ uncertainty ("cyan") band, $\hat{\mu}-\Delta \hat{\mu}$, the central value (bestfit) $\hat{\mu}$, and finally the signal strength modifier at the upper edge of the $1 \sigma$ uncertainty band, $\hat{\mu}+\Delta \hat{\mu}$. In the case of a peak observable definition, as in Table 7 , the data file ends after the 12th row, since the signal strength is only measured at a single Higgs mass value (corresponding to the signal). In contrast, for the construction of mass-centered observables, the data is listed here for the full investigated mass range, which is typically extracted from the corresponding $\hat{\mu}$ plot using EasyNData [102].

As a further remark, we point out a general limitation in the implementation of experimental data: some results from the LHC experiments are given for the combination of data collected at different center-of-mass energies, e.g. at 7 and $8 \mathrm{TeV}$. These results cannot be disentangled by HiggsSignals. Therefore, these observables are implemented as if the data was collected at the center-of-mass energy, which can be assumed to be dominating the experimental data. This approximation is valid, since both the observed and the predicted signal strengths are treated as SM normalized quantities. The only remaining inaccuracy lies in the SM channel weights, Eq. (3), which depend on the center-of-mass energy.

A complication arises in the assignment of Higgs observables if an analysis with one measured mass peak value is split up in several categories, each containing an individual signal rate measurement, see e.g. [84, 103]. In this case, each category result defines a peak observable, however only one of these observables can be associated with the mass measurement from the analysis, which is going to contribute to the $\chi^{2}$. In all other categories this contribution has to be switched off. Nevertheless, this difference in the implementation can lead to inconsistent assignments of the Higgs boson(s) to the category observables. In order to enforce a consistent assignment, peak observables can build an assignment group. This enforces that the Higgs boson(s) are assigned to either all or none of the observables in this group, judged by the assignment status of the observable containing the mass measurement. For each peak observable, the assignment group can be specified in the experimental table, cf. Table 9. Note that the analysis IDs of the category peak observables have to be different from each other.

\section{HiggsSignals applications}

In this section we discuss a few example applications which demonstrate the performance of HiggsSignals. Most of the examples are chosen such that their results can be validated with official results from ATLAS and CMS. The quality of agreement of the reproduced HiggsSignals results with the official results justifies the Gaussian limit approximation in the statistical approach of HiggsSignals. Note that to a certain extent (which is difficult to estimate), the accuracy of the reproduced results suffers from the lack of publicly available information of the analysis efficiencies on the various production modes. At the end of this section, we briefly discuss a few HiggsSignals example applications, where the results incorporate all presently available Higgs data from the LHC and the Tevatron. Another example application of HiggsSignals within the context of the MSSM was presented in Ref. [16]. 


\subsection{Performance studies of HiggsSignals}

\subsubsection{The peak-centered $\chi^{2}$ method for a SM-like Higgs boson}

As a first application we discuss the performance of the peakcentered $\chi^{2}$ method on a SM-like Higgs boson. As already shown in Fig. 1b, a simple one parameter fit can be performed to the signal strength modifier $\mu$, which scales the predicted signal rates of all investigated Higgs channels uniformly. In this fit the Higgs mass is held fixed at e.g. $m_{H}=125.7 \mathrm{GeV}$. Using the signal strength measurements of the individual search channels obtained by the CMS collaboration [77], as given in Fig. 1b, the best-fit signal strength reconstructed with HiggsSignals is $\hat{\mu}_{\text {comb }}=0.77 \pm 0.14$. This agrees well with the official CMS result, $\hat{\mu}_{\text {comb }}^{\mathrm{CMS}}=0.80 \pm 0.14$ [77]. Using HiggsSignals with similar data from ATLAS [104], where the experimental results for all categories are unfortunately not available at a common value for the Higgs mass, the published value of $\hat{\mu}_{\text {comb }}^{\mathrm{ATLAS}}=1.30 \pm 0.20$ at $m_{H}=$ $125.5 \mathrm{GeV}$ [104] is nevertheless reproduced reasonably well by $\hat{\mu}_{\text {comb }}=1.24 \pm 0.20$.

Now, we collect as peak observables the measured signal rates from the LHC experiments ATLAS [75,103-110] and CMS [77,82,84-87,111-116], as well as the Tevatron experiments CDF [117] and DØ [118], as summarized in Fig. 2. If possible, we implement results from the 7 and $8 \mathrm{TeV}$ LHC runs as separate observables. However, if the only quoted result is a combination of both center-of-mass energies we implement it as an $8 \mathrm{TeV}$ result. As mentioned in Sect. 3, we employ the quoted asymmetric uncertainties to account for the dominant effects of potentially remaining non-Gaussian behavior of the measurements. The $H \rightarrow \gamma \gamma$ and $H \rightarrow Z Z^{(*)} \rightarrow 4 \ell$ analyses of ATLAS and CMS have a rather precise mass resolution, thus we treat the implemented mass value of their signal as a measurement which enters the Higgs mass part of the total $\chi^{2}$, cf. Sect. 3.1. Note however that the implemented mass value is not necessarily the most precise measurement of the Higgs mass but rather the mass value for which the signal strength was published by the experimental analysis. The Higgs mass can be determined more accurately from a simultaneous fit to the mass and the signal strength. This can be done with the mass-centered $\chi^{2}$ method, as discussed in the next subsection. Note also that the Higgs mass values assumed in the signal strength measurements can differ by up to $\sim 2.5 \mathrm{GeV}$. It would be desirable if the experiments would present their best-fit signal strengths for all available channels (including specially tagged categories) also for a common Higgs mass (equal or close to the Higgs mass value preferred by the combined data) once a combination of different channels is performed. In the present case, global fits combining the signal strength measurements performed at different Higgs masses rely on the assumption that these measurements do not vary too much within these mass differences.

It can nevertheless be interesting to discuss the total $\chi^{2}$ distribution obtained in the peak-centered $\chi^{2}$ method as a function of the Higgs mass, $m_{H}$. This serves as a demonstration of the three different Higgs mass uncertainty parametrizations (box, Gaussian, box+Gaussian pdfs), as well as the implications of taking into account the correlations among the systematic uncertainties in the $\chi^{2}$ calculation. Furthermore, features of the automatic assignment of the Higgs boson to the peak observables can be studied. In the following example, we set the predicted signal strength for all Higgs channels to their SM values $\left(\mu_{i} \equiv 1\right)$ and set the production and decay rate uncertainties to the values given in Eq. (9), as recommended by the LHC Higgs Cross Section Working Group for the SM Higgs boson around $m_{H} \simeq 125 \mathrm{GeV}$. We then evaluate the total peak-centered $\chi^{2}$ for each Higgs boson mass $m_{H} \in[110,140] \mathrm{GeV}$ using the peak observables presented in Fig. 2. In the SM the Higgs mass is treated as a free parameter, which corresponds to setting the theory mass uncertainty to zero. In order to illustrate the effects of a non-zero theory mass uncertainty, we also consider a model with SM-like Higgs couplings, but which has a $2 \mathrm{GeV}$ theory uncertainty on the predicted Higgs mass.

The total $\chi^{2}$ mass distribution is shown in Fig. 3 for four different cases: In Fig. 3a,b the correlations among the systematic uncertainties of the signal rates, luminosity and Higgs mass predictions are neglected, whereas they are taken into account in Fig. 3c,d. In order to demonstrate the difference between the three parametrizations of the Higgs mass uncertainty we show the $\chi^{2}$ distribution assuming a theoretical Higgs mass uncertainty of $\Delta m=0 \mathrm{GeV}$ in Fig. 3a,c and $\Delta m=2 \mathrm{GeV}$ in Fig. 3b, d, respectively. Furthermore, Fig. 3 includes the number of peak observables, which have been assigned with the Higgs boson, as a function of the Higgs mass. These are depicted by the faint graphs for each Higgs mass uncertainty parametrization.

The discontinuous shape of the $\chi^{2}$ distribution is caused by changes in the Higgs boson assignment to the individual observables. Recall that, if the Higgs mass $m_{H}$ is too far away from the implemented mass position of the peak observable, the Higgs boson is not assigned to the signal. This yields a $\chi^{2}$ contribution corresponding to no predicted signal, $\mu=0$, cf. Sect. 3.1. Most of the peak observables have different mass resolutions, therefore the $\chi^{2}$ distribution has a staircase-like shape. At each step, the total number of peak observable assignments changes.

As can be seen in Fig. 3 all three parametrizations of the theoretical Higgs mass uncertainty yield the same total $\chi^{2}$ values if the Higgs mass $m_{H}$ is far away from the implemented signal mass position, because typically observables which enter the Higgs mass part of the $\chi^{2}$ in the Gaussian parametrization exhibit a decent mass resolution, and the 


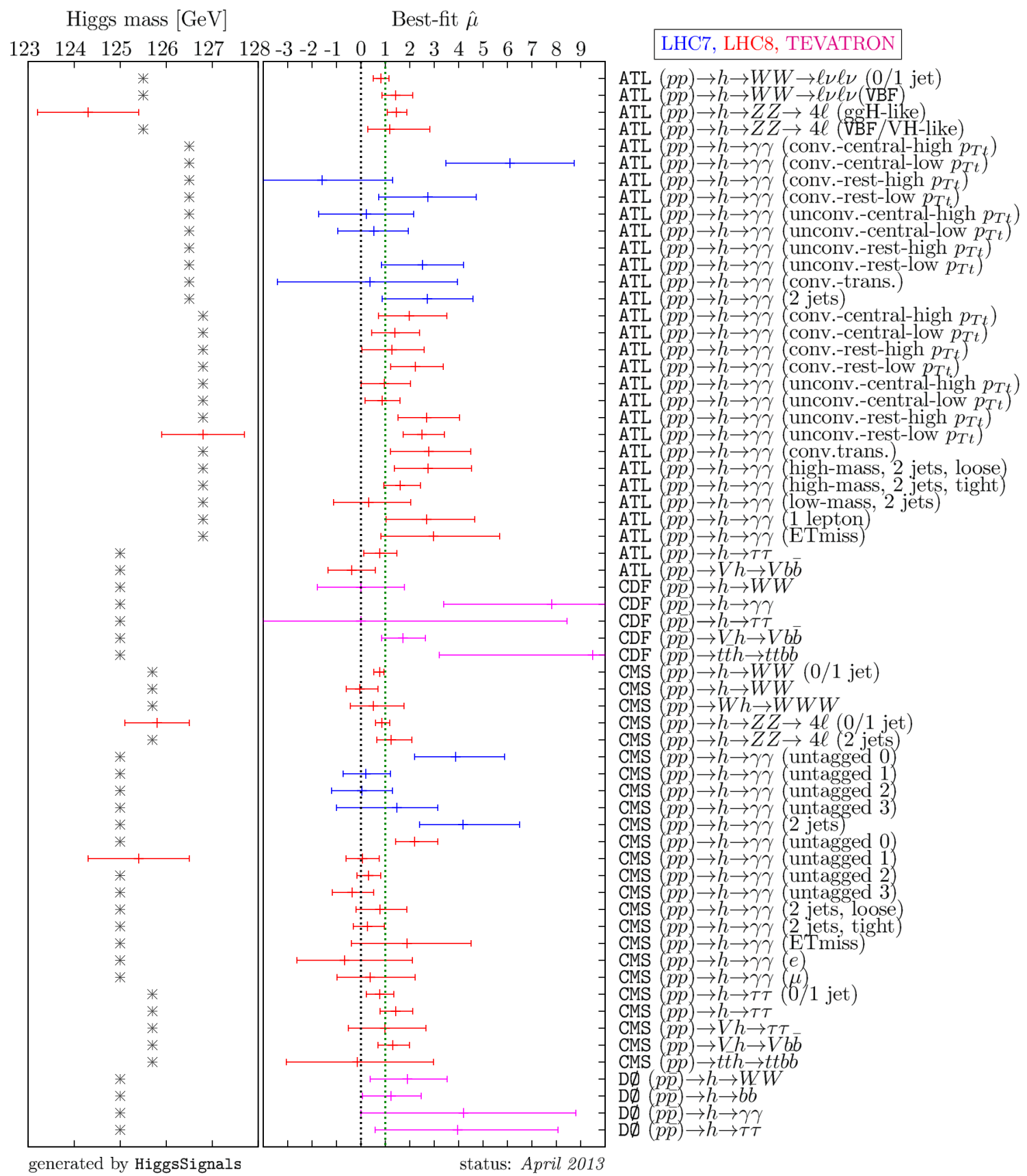

Fig. 2 Overview of the Higgs signal rate and mass measurements (status shortly after the Moriond conference 2013) from ATLAS [75,103110], CMS [77,82,84-87,111-116] and the Tevatron experiments CDF [117] and DØ [118], as they are implemented in HiggsSignals-1.0.0 as peakobservables. The left panel shows the Higgs mass value for which the signal strength was measured. A value with error bars indicates that the mass value is treated as a Higgs mass observable in the peak-centered $\chi^{2}$ method, whereas a gray asterisk only serves as an indication of the Higgs mass value, which was assumed in the rate measurement. This value does not enter directly the total $\chi^{2}$.

Higgs boson is only assigned if this $\chi^{2}$ is low, i.e. $m_{H} \approx \hat{m}$. Conversely, at the $\chi^{2}$ minimum at a Higgs mass $m_{H} \sim 125$ $126 \mathrm{GeV}$, we obtain slightly different $\chi^{2}$ values for the three
For some LHC analyses, measurements for both the 7 and $8 \mathrm{TeV}$ data exist, shown in blue and red, respectively. If the measurement is based on the combined $7 / 8 \mathrm{TeV}$ dataset, we treat it as an $8 \mathrm{TeV}$ measurement only. For the $H \rightarrow \gamma \gamma$ analyses from ATLAS and CMS, the special tagged categories were implemented as separate peak observables, including their efficiencies, but collected together in assignment groups. In total there are 4 Higgs mass observables and 63 Higgs signal rate observables. This data is used for the performance scans in Fig. 3 and the example applications in Sect. 5.3

parametrizations: Firstly, assuming that every observable is assigned with the Higgs boson, the minimal $\chi^{2}$ is in general slightly higher in the Gaussian case than in the box and 

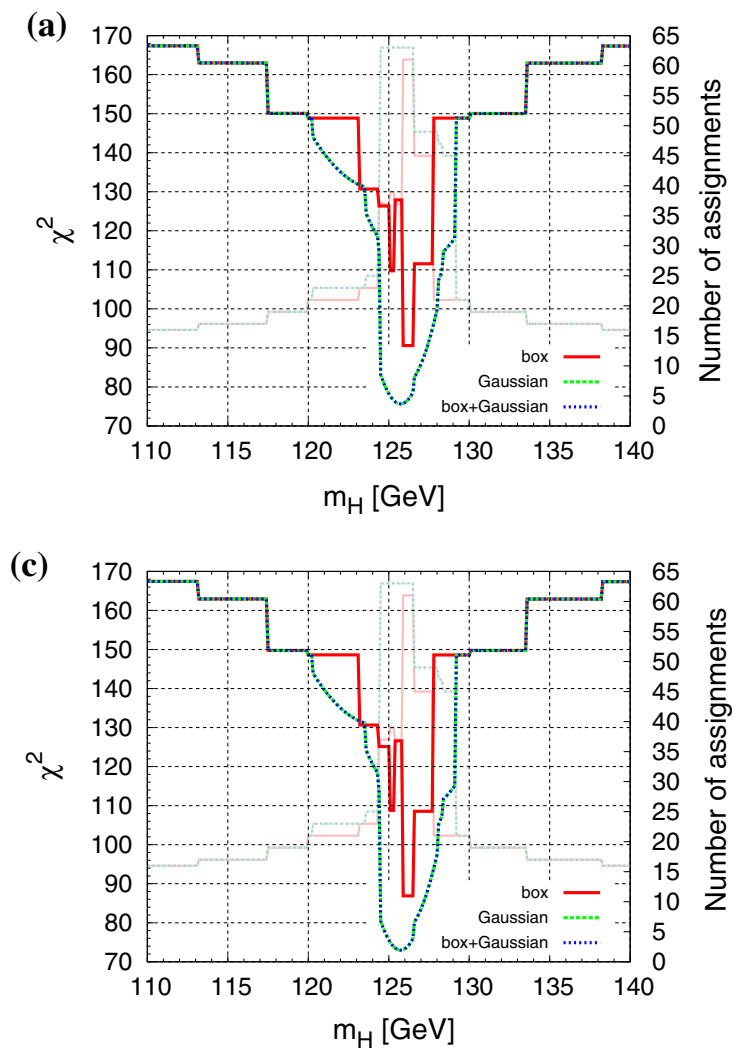

Fig. 3 Total $\chi^{2}$ distribution obtained by the peak-centered $\chi^{2}$ method for a SM Higgs boson with mass $m_{H}$ obtained from the 63 peak observables (status: April 2013) shown in Fig. 2. In (a, b), the total $\chi^{2}$ is evaluated without taking into account the correlations among the systematic uncertainties, whereas they are fully included in $(\mathbf{c}, \mathbf{d})$. In $(\mathbf{a}, \mathbf{c})$ no theoretical mass uncertainty $\Delta m$ is assumed (like in the SM) whereas in (b, d) we set $\Delta m=2 \mathrm{GeV}$. For each setting, we show the total $\chi^{2}$ obtained

box+Gaussian case if the Higgs mass measurements do not have the same central values for all (mass sensitive) peak observables. In that case, there will always be a non-zero $\chi^{2}$ contribution from the Higgs mass measurements for any predicted value of the Higgs mass. Secondly, in the case of no theoretical mass uncertainty, the box parametrization does not exhibit a full assignment of all currently implemented peak observables at any Higgs mass value. This is because the mass measurements of the ATLAS $H \rightarrow \gamma \gamma$ [103] and $H \rightarrow Z Z^{(*)} \rightarrow 4 \ell[75]$ observables have a mass difference of $2.5 \mathrm{GeV}$, which corresponds to a discrepancy of around $2.5 \sigma$ [119]. Thus, the Higgs boson is only assigned to either of these (groups of) observables, receiving a maximal $\chi^{2}$ penalty from the other observable (group). In fact, we observe a double minimum structure in Fig. 3a,c, because for a Higgs mass $m_{H} \in[125.4,125.8] \mathrm{GeV}$, neither the ATLAS $H \rightarrow \gamma \gamma$ nor the $H \rightarrow Z Z^{(*)} \rightarrow 4 \ell$ observables are assigned with the Higgs boson, leading to a large total $\chi^{2}$. This illustrates that the box-shaped pdf is an inappropriate description of the Higgs mass likelihood in the absence of sizable theoretical mass uncertainties.
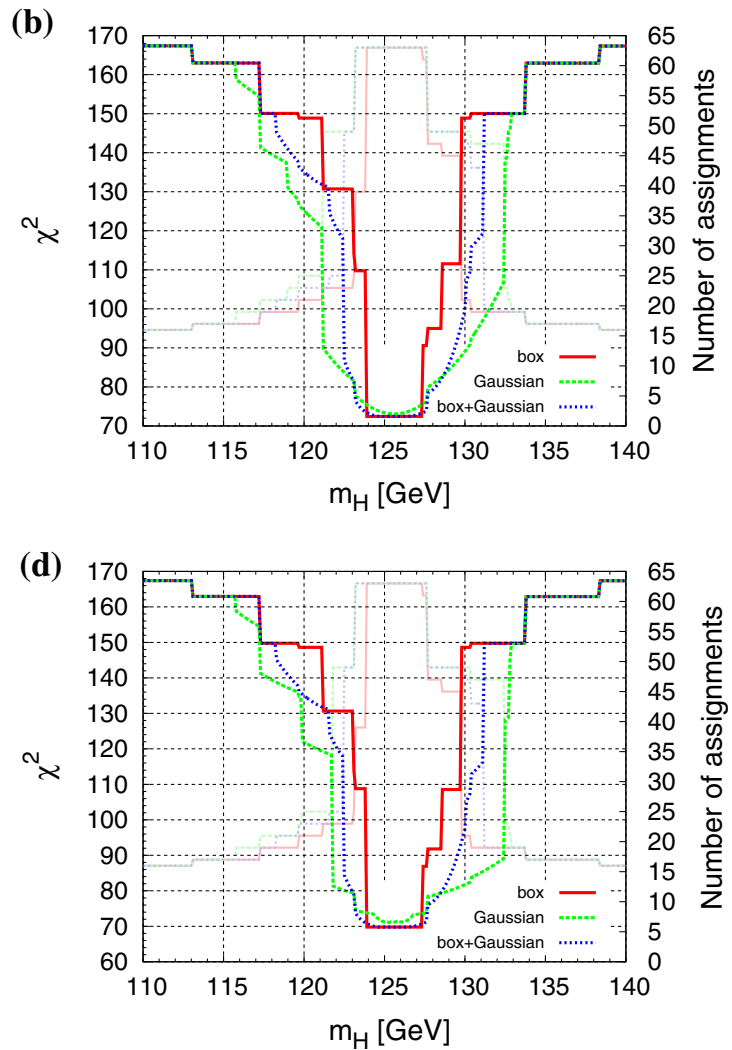

for all three parametrizations of the theoretical Higgs mass uncertainty: box (solid red), Gaussian (dashed green) and box+Gaussian (dotted blue) pdf. For each case, we also give the total number of peak observables, which have been assigned with the Higgs boson, depicted by the corresponding faint lines. a No correlations, $\Delta m=0 \mathrm{GeV}$. b No correlations, $\Delta m=2 \mathrm{GeV}$. c With correlations, $\Delta m=0 \mathrm{GeV}$. d With correlations, $\Delta m=2 \mathrm{GeV}$

A difference between the Gaussian and the theory box with experimental Gaussian (box+Gaussian) parametrization appears only for non-zero $\Delta m$. For $\Delta m=2 \mathrm{GeV}$ the minimal $\chi^{2}$ is obtained for a plateau $m_{H} \approx(124.8-126.5) \mathrm{GeV}$ in the box+Gaussian case, whereas in the Gaussian case we have a non-degenerate minimum at $m_{H}=125.7 \mathrm{GeV}$. However, outside this plateau the $\chi^{2}$ shape of the box+Gaussian increases faster than in the Gaussian case, since the uncertainty governing this Gaussian slope is smaller.

For the Gaussian parametrization of the theoretical Higgs mass uncertainty and no theoretical mass uncertainty the minimal $\chi^{2}$ at $m_{H}=125.7 \mathrm{GeV}$ changes from 75.7 to 73.0 (for 63 signal strength observables and 4 mass observables) if we include the correlations among the systematic uncertainties in the $\chi^{2}$ evaluation. In the case of a non-zero theoretical mass uncertainty, also the shape of the total $\chi^{2}$ distribution can be affected when the correlations are taken into account. Recall that only in the Gaussian parametrization the correlations of the theoretical mass uncertainties enter the $\chi^{2}$ evaluation, featuring a sign dependence on the relative position of the predicted Higgs mass value with respect to the two 
(a)

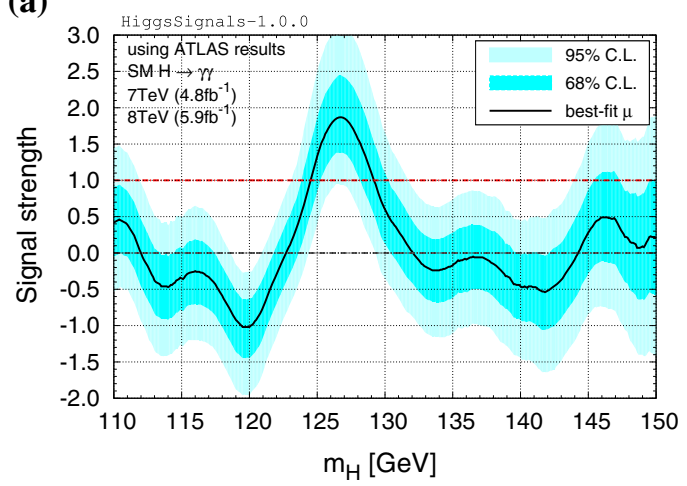

(c)

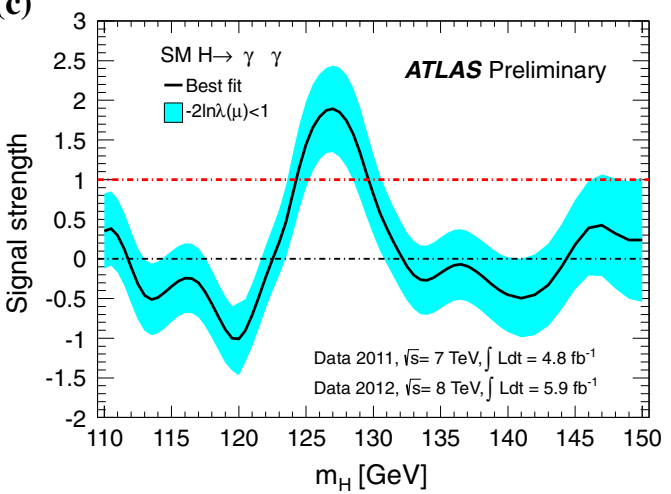

Fig. 4 Reconstruction of the combined best-fit signal strength from the results of the individual dataset / channels with the mass-centered $\chi^{2}$ method (a, b). For comparison, we give the official ATLAS results in (c, d). a Simultaneous evaluation of 7 and $8 \mathrm{TeV}$ results from the ATLAS SM $H \rightarrow \gamma \gamma$ search [105]. b Simultaneous evaluation of

observed Higgs mass values, cf. Section 3.1.2. This results in a shallower slope of the $\chi^{2}$ distribution at Higgs masses larger than all mass measurements, $m_{H} \gtrsim 126.8 \mathrm{GeV}$, since all mass observables are positively correlated in this case.

In conclusion we would like to emphasize that, although the direct $\chi^{2}$ contribution from (the few) mass measurements to the total $\chi^{2}$ might appear small in comparison to the $\chi^{2}$ contribution from (many) signal strength measurements, the automatic assignment of Higgs boson(s) to the peak observables introduces a strong mass dependence, even for peak observables without an implemented mass measurement. Hereby, the procedure tries to ensure that a comparison of the predicted and observed signal strength is valid for each observable (depending on the mass resolution of the corresponding Higgs analysis), or otherwise considers the signal as not explainable by the model.

\subsubsection{Combining search channels with the mass-centered $\chi^{2}$ method}

As a first demonstration of the mass-centered $\chi^{2}$ method we evaluate simultaneously the 7 and $8 \mathrm{TeV}$ results from ATLAS (b)

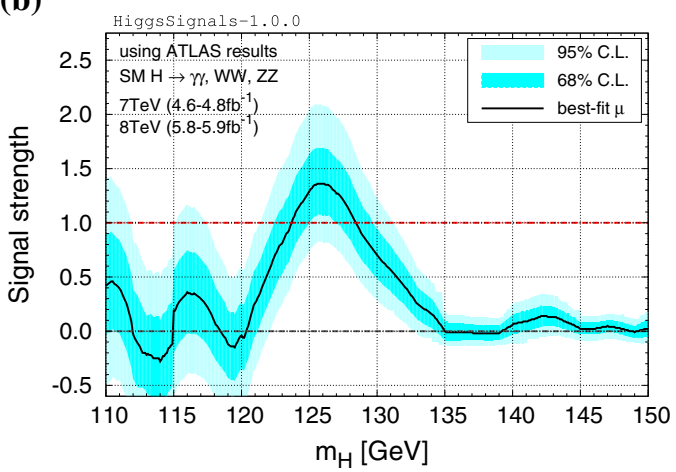

(d)

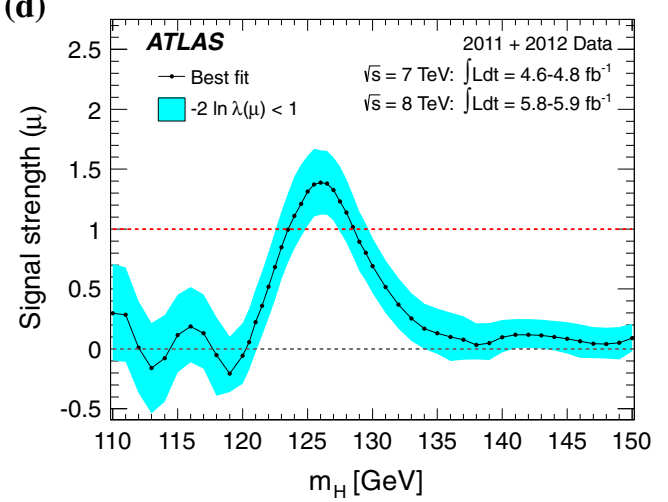

ATLAS searches for $H \rightarrow \gamma \gamma, Z Z$ and $W W$ [105,120,121]. c Official ATLAS combination of 7 and $8 \mathrm{TeV}$ results from the ATLAS SM $H \rightarrow \gamma \gamma$ search [105]. d Official ATLAS combination of the SM $H \rightarrow \gamma \gamma, Z Z, W W, b \bar{b}$ and $\tau^{+} \tau^{-}$searches [17]

for the Higgs searches $H \rightarrow \gamma \gamma$ [105], as well as its evaluation together with the $H \rightarrow W W^{(*)} \rightarrow \ell \nu \ell v$ [120] and $H \rightarrow Z Z^{(*)} \rightarrow 4 \ell$ [121] searches. This is possible because the full $\hat{\mu}$ plot was published for these analyses for 7 and $8 \mathrm{TeV}$, except for the $H \rightarrow Z Z^{(*)} \rightarrow 4 \ell$ search where only the combined $7 / 8 \mathrm{TeV}$ result is available. ${ }^{18}$

We scan the relevant Higgs mass range $m_{H}=(110-$ 150) $\mathrm{GeV}$, as well as the signal strength $\mu$, and at each point $\left(m_{H}, \mu\right)$ evaluate the mass-centered $\chi^{2}$ using the corresponding $\hat{\mu}$ plots as mass-centered observables. We then find the best-fit $\mu$ value (and the corresponding $1 \sigma$ and $2 \sigma$ regions) by minimizing the $\chi^{2}$ (finding $\Delta \chi^{2}=1$ and $\Delta \chi^{2}=4$, respectively) for a fixed Higgs mass $m_{H}$. This is shown in Fig. 4a and b for the $H \rightarrow \gamma \gamma$ channel and the combination of $H \rightarrow \gamma \gamma, H \rightarrow W W^{(*)} \rightarrow \ell \nu \ell v$ and $H \rightarrow Z Z^{(*)} \rightarrow 4 \ell$, respectively. These results nicely agree with the corresponding official ATLAS results [17,105], which are shown in Fig. 4c,d for comparison. Especially at the signal around $\simeq 126 \mathrm{GeV}$ the Gaussian limit approxima-

\footnotetext{
18 Since it is not possible to disentangle this result into 7 and $8 \mathrm{TeV}$, we implemented this observable as $8 \mathrm{TeV}$ only data in Higgs Signals.
} 




Fig. 5 Results from a simultaneous fit to the Higgs mass and signal strength using the experimental data from the ATLAS searches $H \rightarrow$ $\gamma \gamma$ [122], $H \rightarrow W W^{(*)} \rightarrow \ell \nu \ell \nu$ [109] and $H \rightarrow Z Z^{(*)} \rightarrow 4 \ell$ [75]. The corresponding results from ATLAS are overlaid as faintly colored contours

tion works very well due to the relatively large number of events (in the $H \rightarrow \gamma \gamma$ analysis). Note that in Fig. 4d also the channels $H \rightarrow \tau \tau$ and $V H \rightarrow b \bar{b}$ are included, however, these observables are rather insignificant for this result due to large uncertainties on the signal strength measurement as well as a poor mass resolution.

Instead of minimizing the $\chi^{2}$ for a fixed Higgs mass $m_{H}$, we now perform a two parameter fit to $m_{H}$ and $\mu$, using the latest currently available $\hat{\mu}$ plots from the ATLAS searches ${ }^{19}$ $H \rightarrow \gamma \gamma$ [122], $H \rightarrow W W^{(*)} \rightarrow \ell \nu \ell \nu$ [109] and $H \rightarrow$ $Z Z^{(*)} \rightarrow 4 \ell$ [75]. For a given signal hypothesis, $\left(m_{H}, \mu\right)$, we scan the full mass range, $m_{H}^{\prime} \in[115,150] \mathrm{GeV}$ with a step size of $0.1 \mathrm{GeV}$, and the signal strength modifier $\mu^{\prime}$ in steps of 0.05 . For each scanning point we evaluate the masscentered $\chi^{2}$ value, $\chi_{\mathrm{MC}}^{2}$, for the hypothesis $\left(m_{H}^{\prime}, \mu^{\prime}\right)$, where

$\mu^{\prime}=\left\{\begin{array}{lll}\mu & \text { if } \quad m_{H}^{\prime}=m_{H} \\ 0 & \text { if } \quad m_{H}^{\prime} \neq m_{H}\end{array}\right.$

The obtained $\chi^{2}$ values from this scan are summed and associated with the point $\left(m_{H}, \mu\right)$. Thus we test the combined hypothesis of having a Higgs boson at $m_{H}$ with signal strength $\mu$, and no signal elsewhere. The procedure is then repeated for all points in the two-dimensional $\left(m_{H}, \mu\right)$ plane to obtain the 2D $\chi^{2}$ likelihood map. The results are shown in Fig. 5 for each Higgs decay mode separately. For comparison, we also show the official ATLAS results [109,110,122] as faintly colored contours. Qualitatively, the obtained 68 and $95 \%$ C.L. regions (corresponding to $\Delta \chi^{2}=2.30$ and

\footnotetext{
19 ATLAS did not include a new $\hat{\mu}$ plot in their $H \rightarrow \gamma \gamma$ search update at the Moriond 2013 conference [103]. Therefore, we have to use an older result here. We use the $\hat{\mu}$ plot from [122] which includes the mass scale systematic (MSS) uncertainty.
}

$\Delta \chi^{2}=5.99$, respectively) agree fairly well for $H \rightarrow Z Z$ and $H \rightarrow W W$, whereas the $H \rightarrow \gamma \gamma$ result is shifted towards larger Higgs masses by around $0.8 \mathrm{GeV}$. A potential reason for this discrepancy is that effects of the mass scale systematic (MSS) uncertainty are only indirectly taken into account in HiggsSignals by simply using the corresponding plateau-shaped $\hat{\mu}$ plot [122] instead of including the MSS uncertainty in the profile likelihood as a nuisance. Nevertheless, the 68 and $95 \%$ C.L. regions still have a large overlap. Note also that the spiky structures of the contour ellipses in Fig. 5 are rather an artifact of our data extraction with EasyNData [102] than a physical effect. ${ }^{20}$

A simultaneous fit to the ATLAS Higgs channels $H \rightarrow$ $\gamma \gamma$ [122], $H \rightarrow Z Z^{(*)} \rightarrow 4 \ell$ [75] and $H \rightarrow W W^{(*)} \rightarrow$ $\ell \nu \ell v[109]$ can also be performed. The best fit point of such a combination is found at

$m_{H}=125.4_{-0.4}^{+0.2} \mathrm{GeV}, \mu=1.4_{-0.2}^{+0.3}$,

where the uncertainties given refer to the 1D profiled $68 \%$ confidence interval. We have verified that these results remain stable when varying the step sizes in the scan.

The two discussed examples show the usefulness of the mass-centered $\chi^{2}$ method. We focussed here on the validation of the method by comparing with official results from ATLAS. It is however easy to go beyond that and take all available data from ATLAS and CMS (and the Tevatron) into account for a simultaneous analysis. This we leave for a future study. However, we would like to emphasize again that the usefulness of this method strongly depends on the information (here in particular the $\hat{\mu}$ plots for the individual channels) the experimental collaborations decide to publish.

\subsection{Validation with official fit results for Higgs coupling scaling factors}

A major task after the discovery of a Higgs-like state is the determination of its coupling properties and thus a thorough test of its compatibility with the SM. Both ATLAS $[104,123]$ and CMS [77,115] have obtained results for Higgs coupling scaling factors in the framework of restricted benchmark models proposed by the LHC Higgs Cross Section Working Group [101]. Numerous other studies have been performed, both for Higgs coupling scaling factors [20-48] as well as for particular models, including composite Higgs scenarios [49-52], Two Higgs Doublet Models (2HDMs) [53-57], supersymmetric models [58-66] as well as other, more exotic extensions of the SM $[67,68]$. Here, we want to focus on the reproduction of the official ATLAS and CMS results using

\footnotetext{
${ }^{20}$ It would therefore be desirable if the experimental collaborations published the data of the $\hat{\mu}$ plots also in tabular form in accurate precision.
} 
Table 10 Signal strength measurements, $\hat{\mu}$, from various ATLAS Higgs searches implemented in HiggsSignals as peak observables

\begin{tabular}{|c|c|c|c|c|c|c|c|}
\hline \multirow[t]{2}{*}{ Higgs search channel } & \multirow[t]{2}{*}{ Energy $\sqrt{s}(\mathrm{TeV})$} & \multirow[t]{2}{*}{$\hat{\mu} \pm \Delta \hat{\mu}$} & \multicolumn{5}{|c|}{ SM signal composition (\%) } \\
\hline & & & $\mathrm{ggH}$ & VBF & WH & $\mathrm{ZH}$ & $t \bar{t} H$ \\
\hline$H \rightarrow W W^{(*)} \rightarrow \ell \nu \ell v(0 / 1$ jet $)[109,110]$ & $7 / 8$ & $0.82_{-0.32}^{+0.33}$ & 97.2 & 1.6 & 0.7 & 0.4 & 0.1 \\
\hline$H \rightarrow W W^{(*)} \rightarrow \ell \nu \ell v(2$ jet $)[109,110]$ & $7 / 8$ & $1.42_{-0.56}^{+0.70}$ & 19.8 & 80.2 & 0.0 & 0.0 & 0.0 \\
\hline$H \rightarrow Z Z^{(*)} \rightarrow 4 \ell($ ggH-like $)[75,110]$ & $7 / 8$ & $1.45_{-0.37}^{+0.43}$ & 92.5 & 4.5 & 1.9 & 1.1 & 0.0 \\
\hline$H \rightarrow Z Z^{(*)} \rightarrow 4 \ell(\mathrm{VBF} / \mathrm{VH}-\mathrm{like})[75,110]$ & $7 / 8$ & $1.18_{-0.90}^{+1.64}$ & 36.8 & 43.1 & 12.8 & 7.3 & 0.0 \\
\hline$H \rightarrow \gamma \gamma$ (unconv.-central-low $\left.p_{T t}\right)[105]$ & 7 & $0.53_{-1.48}^{+1.41}$ & 92.9 & 3.8 & 2.0 & 1.1 & 0.2 \\
\hline$H \rightarrow \gamma \gamma$ (unconv.-central-high $p_{T t}$ ) [105] & 7 & $0.22_{-1.95}^{+1.94}$ & 65.5 & 14.8 & 10.8 & 6.2 & 2.7 \\
\hline$H \rightarrow \gamma \gamma$ (unconv.-rest-low $p_{T t}$ ) [105] & 7 & $2.52_{-1.68}^{+1.68}$ & 92.6 & 3.7 & 2.2 & 1.2 & 0.2 \\
\hline$H \rightarrow \gamma \gamma$ (unconv.-rest-high $p_{T t}$ ) [105] & 7 & $10.44_{-3.70}^{+3.67}$ & 64.4 & 15.2 & 11.8 & 6.6 & 2.0 \\
\hline$H \rightarrow \gamma \gamma\left(\right.$ conv.-central-low $\left.p_{T t}\right)[105]$ & 7 & $6.10_{-2.62}^{+2.63}$ & 92.7 & 3.8 & 2.1 & 1.1 & 0.2 \\
\hline$H \rightarrow \gamma \gamma\left(\right.$ conv.-central-high $\left.p_{T t}\right)$ [105] & 7 & $-4.36_{-1.81}^{+1.80}$ & 65.7 & 14.4 & 11.0 & 6.2 & 2.8 \\
\hline$H \rightarrow \gamma \gamma$ (conv.-rest-low $\left.p_{T t}\right)[105]$ & 7 & $2.74_{-2.01}^{+1.98}$ & 92.7 & 3.6 & 2.2 & 1.2 & 0.2 \\
\hline$H \rightarrow \gamma \gamma$ (conv.-rest-high $\left.p_{T t}\right)$ [105] & 7 & $-1.59_{-2.90}^{+2.89}$ & 64.4 & 15.1 & 12.1 & 6.4 & 2.0 \\
\hline$H \rightarrow \gamma \gamma$ (conv.-trans.) [105] & 7 & $0.37_{-3.79}^{+3.58}$ & 89.2 & 5.0 & 3.7 & 1.9 & 0.3 \\
\hline$H \rightarrow \gamma \gamma(2$ jet $)[105]$ & 7 & $2.72_{-1.85}^{+1.87}$ & 23.3 & 75.9 & 0.5 & 0.2 & 0.1 \\
\hline$H \rightarrow \gamma \gamma\left(\right.$ unconv.-central-low $\left.p_{T t}\right)$ [103] & 8 & $0.87_{-0.70}^{+0.73}$ & 92.0 & 5.0 & 1.7 & 0.8 & 0.5 \\
\hline$H \rightarrow \gamma \gamma$ (unconv.-central-high $\left.p_{T t}\right)$ [103] & 8 & $0.96_{-0.95}^{+1.07}$ & 78.6 & 12.6 & 4.7 & 2.6 & 1.4 \\
\hline$H \rightarrow \gamma \gamma$ (unconv.-rest-low $p_{T t}$ ) [103] & 8 & $2.50_{-0.77}^{+0.92}$ & 92.0 & 5.0 & 1.7 & 0.8 & 0.5 \\
\hline$H \rightarrow \gamma \gamma$ (unconv.-rest-high $p_{T t}$ ) [103] & 8 & $2.69_{-1.17}^{+1.35}$ & 78.6 & 12.6 & 4.7 & 2.6 & 1.4 \\
\hline$H \rightarrow \gamma \gamma\left(\right.$ conv.-central-low $\left.p_{T t}\right)[103]$ & 8 & $1.39_{-0.95}^{+1.01}$ & 92.0 & 5.0 & 1.7 & 0.8 & 0.5 \\
\hline$H \rightarrow \gamma \gamma\left(\right.$ conv.-central-high $\left.p_{T t}\right)[103]$ & 8 & $1.98_{-1.26}^{+1.54}$ & 78.6 & 12.6 & 4.7 & 2.6 & 1.4 \\
\hline$H \rightarrow \gamma \gamma\left(\right.$ conv.-rest-low $\left.p_{T t}\right)[103]$ & 8 & $2.23_{-1.01}^{+1.14}$ & 92.0 & 5.0 & 1.7 & 0.8 & 0.5 \\
\hline$H \rightarrow \gamma \gamma\left(\right.$ conv.-rest-high $\left.p_{T t}\right)[103]$ & 8 & $1.27_{-1.23}^{+1.32}$ & 78.6 & 12.6 & 4.7 & 2.6 & 1.4 \\
\hline$H \rightarrow \gamma \gamma$ (conv.trans.) $[103]$ & 8 & $2.78_{-1.57}^{+1.72}$ & 92.0 & 5.0 & 1.7 & 0.8 & 0.5 \\
\hline$H \rightarrow \gamma \gamma$ (high-mass, 2 jet, loose) [103] & 8 & $2.75_{-1.38}^{+1.78}$ & 45.3 & 53.7 & 0.5 & 0.3 & 0.2 \\
\hline$H \rightarrow \gamma \gamma$ (high-mass, 2 jet, tight) $[103]$ & 8 & $1.61_{-0.67}^{+0.83}$ & 27.1 & 72.5 & 0.3 & 0.1 & 0.0 \\
\hline$H \rightarrow \gamma \gamma$ (low-mass, 2 jet) [103] & 8 & $0.32_{-1.44}^{+1.72}$ & 38.0 & 2.9 & 40.1 & 16.9 & 2.1 \\
\hline$H \rightarrow \gamma \gamma\left(E_{T}^{\text {miss }}\right.$ sign. $)[103]$ & 8 & $2.97_{-2.15}^{+2.71}$ & 4.4 & 0.3 & 35.8 & 47.4 & 12.2 \\
\hline$H \rightarrow \gamma \gamma(1 \ell)[103]$ & 8 & $2.69_{-1.66}^{+1.97}$ & 2.5 & 0.4 & 63.3 & 15.2 & 18.7 \\
\hline$H \rightarrow \tau \tau[104,106]$ & $7 / 8$ & $0.77_{-0.65}^{+0.70}$ & 88.1 & 7.1 & 3.1 & 1.7 & 0.0 \\
\hline$V H \rightarrow V(b b)[104,107]$ & $7 / 8$ & $-0.38_{-0.97}^{+0.97}$ & 0.0 & 0.0 & 63.8 & 36.2 & 0.0 \\
\hline
\end{tabular}

Results from combined $7 / 8 \mathrm{TeV}$ data are implemented as $8 \mathrm{TeV}$-only in HiggsSignals. The $H \rightarrow \gamma \gamma$ measurements where performed at a Higgs mass of $m_{H}=126.5 \mathrm{GeV}[126.8 \mathrm{GeV}]$ for the $7 \mathrm{TeV}[8 \mathrm{TeV}]$ results, while the remaining channels are measured at $m_{H}=125.5 \mathrm{GeV}$. In the last columns, we give the assumed signal composition for a SM Higgs boson

the Higgs coupling scaling factors as defined in the benchmark models of Ref. [101] in order to validate the Higgs Signals implementation.
We validate with the ATLAS and CMS results, as presented at the Moriond 2013 conference [104,115]. The measurements from ATLAS and CMS, which are used as observ- 
Table 11 Signal strength measurements, $\hat{\mu}$, from various CMS Higgs searches implemented in HiggsSignals as peak observables

\begin{tabular}{|c|c|c|c|c|c|c|c|}
\hline \multirow[t]{2}{*}{ Higgs search channel } & \multirow[t]{2}{*}{ Energy $\sqrt{s}(\mathrm{TeV})$} & \multirow[t]{2}{*}{$\hat{\mu} \pm \Delta \hat{\mu}$} & \multicolumn{5}{|c|}{ SM signal composition (\%) } \\
\hline & & & $\operatorname{ggH}$ & VBF & WH & $\mathrm{ZH}$ & $t \bar{t} H$ \\
\hline$H \rightarrow W W^{(*)} \rightarrow \ell \nu \ell \nu(0 / 1$ jet $)[87]$ & $7 / 8$ & $0.77_{-0.24}^{+0.17}$ & 95.0 & 5.0 & 0.0 & 0.0 & 0.0 \\
\hline$H \rightarrow W W^{(*)} \rightarrow \ell v \ell v(\mathrm{VBF})[111,112]$ & $7 / 8$ & $-0.05_{-0.55}^{+0.75}$ & 38.2 & 61.8 & 0.0 & 0.0 & 0.0 \\
\hline$W H \rightarrow W\left(W W^{(*)}\right) \rightarrow 3 \ell 3 v[124]$ & $7 / 8$ & $0.51_{-0.94}^{+1.26}$ & 0.0 & 0.0 & $100.0^{\mathrm{a}}$ & 0.0 & 0.0 \\
\hline$H \rightarrow Z Z^{(*)} \rightarrow 4 \ell(0 / 1$ jet $)[82]$ & $7 / 8$ & $0.86_{-0.26}^{+0.32}$ & 89.8 & 10.2 & 0.0 & 0.0 & 0.0 \\
\hline$H \rightarrow Z Z^{(*)} \rightarrow 4 \ell(2$ jet $)[82]$ & $7 / 8$ & $1.24_{-0.58}^{+0.85}$ & 71.2 & 28.8 & 0.0 & 0.0 & 0.0 \\
\hline$H \rightarrow \gamma \gamma($ untagged 0$)[84,113]$ & 7 & $3.88_{-1.68}^{+2.00}$ & 61.4 & 16.9 & 12.0 & 6.6 & 3.1 \\
\hline$H \rightarrow \gamma \gamma($ untagged 1) $[84,113]$ & 7 & $0.20_{-0.93}^{+1.01}$ & 87.7 & 6.2 & 3.6 & 2.0 & 0.5 \\
\hline$H \rightarrow \gamma \gamma($ untagged 2$)[84,113]$ & 7 & $0.04_{-1.24}^{+1.25}$ & 91.4 & 4.4 & 2.5 & 1.4 & 0.3 \\
\hline$H \rightarrow \gamma \gamma($ untagged 3) $[84,113]$ & 7 & $1.47_{-2.47}^{+1.68}$ & 91.3 & 4.4 & 2.6 & 1.5 & 0.2 \\
\hline$H \rightarrow \gamma \gamma(2$ jet $)[84,113]$ & 7 & $4.18_{-1.78}^{+2.31}$ & 26.7 & 72.6 & 0.4 & 0.2 & 0.0 \\
\hline$H \rightarrow \gamma \gamma($ untagged 0) [84] & 8 & $2.20_{-0.78}^{+0.95}$ & 72.9 & 11.7 & 8.2 & 4.6 & 2.6 \\
\hline$H \rightarrow \gamma \gamma($ untagged 1) [84] & 8 & $0.06_{-0.67}^{+0.69}$ & 83.5 & 8.5 & 4.5 & 2.6 & 1.0 \\
\hline$H \rightarrow \gamma \gamma$ (untagged 2) [84] & 8 & $0.31_{-0.47}^{+0.50}$ & 91.5 & 4.5 & 2.3 & 1.3 & 0.4 \\
\hline$H \rightarrow \gamma \gamma$ (untagged 3) [84] & 8 & $-0.36_{-0.81}^{+0.88}$ & 92.5 & 3.9 & 2.1 & 1.2 & 0.3 \\
\hline$H \rightarrow \gamma \gamma(2$ jet, tight $)[84]$ & 8 & $0.27_{-0.58}^{+0.69}$ & 20.6 & 79.0 & 0.2 & 0.1 & 0.1 \\
\hline$H \rightarrow \gamma \gamma(2$ jet, loose $)[84]$ & 8 & $0.78_{-0.98}^{+1.10}$ & 46.8 & 51.1 & 1.1 & 0.6 & 0.5 \\
\hline$H \rightarrow \gamma \gamma(\mu)[84]$ & 8 & $0.38_{-1.36}^{+1.84}$ & 0.0 & 0.2 & 50.4 & 28.6 & 20.8 \\
\hline$H \rightarrow \gamma \gamma(e)[84]$ & 8 & $-0.67_{-1.95}^{+2.78}$ & 1.1 & 0.4 & 50.2 & 28.5 & 19.8 \\
\hline$H \rightarrow \gamma \gamma\left(E_{T}^{\mathrm{miss}}\right)[84]$ & 8 & $1.89_{-2.28}^{+2.62}$ & 22.1 & 2.6 & 40.6 & 23.0 & 11.7 \\
\hline$H \rightarrow \tau \tau(0 / 1$ jet $)[86]$ & $7 / 8$ & $0.77_{-0.55}^{+0.58}$ & 95.0 & 5.0 & 0.0 & 0.0 & 0.0 \\
\hline$H \rightarrow \tau \tau(\mathrm{VBF})[86]$ & $7 / 8$ & $1.42_{-0.64}^{+0.70}$ & 19.8 & 80.2 & 0.0 & 0.0 & 0.0 \\
\hline$V H \rightarrow V(\tau \tau)[86,125]$ & $7 / 8$ & $0.98_{-1.50}^{+1.68}$ & 0.0 & 0.0 & 17.2 & 9.8 & 0.0 \\
\hline$V H \rightarrow V(b b)[84,85]$ & $7 / 8$ & $1.30_{-0.63}^{+0.73}$ & 0.0 & 0.0 & 63.8 & 36.2 & 0.0 \\
\hline$t t H \rightarrow t t(b b)[116]$ & $7 / 8$ & $-0.15_{-2.90}^{+3.12}$ & 0.0 & 0.0 & 0.0 & 0.0 & 100.0 \\
\hline
\end{tabular}

a The signal is contaminated to $12.0 \%$ by $W H \rightarrow W(\tau \tau)$

Results from combined $7 / 8 \mathrm{TeV}$ data are implemented as $8 \mathrm{TeV}$-only in HiggsSignals. The $H \rightarrow \gamma \gamma$ measurements where performed at a Higgs mass of $m_{H}=125.0 \mathrm{GeV}$, while the remaining channels are measured at $m_{H}=125.7 \mathrm{GeV}$. In the last columns, we give the assumed signal composition for a SM Higgs boson

ables for our reproduced fits, are summarized in Tables 10 and 11, respectively. In the ATLAS fits of Higgs coupling scaling factors the Higgs mass is assumed to be $m_{H}=$ 125.5 GeV. However, for a Higgs mass of $125.5 \mathrm{GeV}$ there are no signal strengths measurements for the $H \rightarrow \gamma \gamma$ categories available in the literature. Instead, we use the $\hat{\mu}$ measurements performed at 126.5 and $126.8 \mathrm{GeV}$ for the 7 and $8 \mathrm{TeV}$ data, respectively [103,105], keeping in mind that this might lead to some inaccuracies. The ATLAS $H \rightarrow W W^{(*)} \rightarrow \ell \nu \ell \nu$ and $H \rightarrow Z Z^{(*)} \rightarrow 4 \ell$ signal strength measurements were extracted from Ref. [110]. Note that for the remaining channels, $H \rightarrow \tau \tau$ and $V H \rightarrow V b \bar{b}$, only the inclusive $\hat{\mu}$ measurements are available in the literature, whereas the ATLAS fit also includes information of their sub-channels [104]. In the CMS fits of Higgs coupling scaling factors a Higgs mass of $m_{H}=125.7 \mathrm{GeV}$ is assumed. All signal strength measurements, as listed in Table 11, have been performed for this assumed Higgs mass value, except for the $H \rightarrow \gamma \gamma$ categories being measured at $m_{H}=125.0 \mathrm{GeV}$.

Before we discuss the benchmark fits of Higgs coupling scale factors, we look at ATLAS and CMS fits that explicitly target the different production modes by combining channels with a particular decay mode. These fits allow to investigate sources of potential deviations between the official and 
(a)

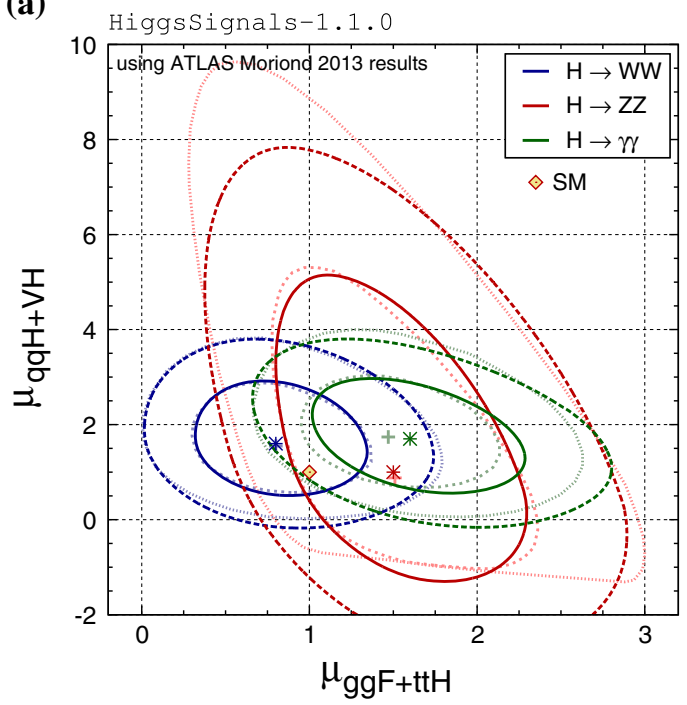

Fig. 6 Comparison of fit results for the universal scale factors for the production cross sections of gluon-gluon fusion (ggf) and top quark pair associated Higgs production ( $\mathrm{ttH}), \mu_{\mathrm{ggf}+\mathrm{ttH}}$, and of vector boson fusion $(\mathrm{qqH})$ and vector boson associated Higgs production $(\mathrm{VH}), \mu_{\mathrm{qqH}}+\mathrm{VH}$, using the individual Higgs search channel results from ATLAS [in a] and CMS [in b]. The $68 \%$ (95\%) C.L. regions are shown as deep colored, solid (dashed) and faintly colored, dotted (fine-dotted) con-

the reproduced HiggsSignals results separately for each Higgs boson decay mode. Furthermore, unknown channel efficiencies can be adjusted within reasonable ranges, such that the agreement of the fit outcome is optimized. The signal composition of all included observables after this optimization is given in Tables 10 and 11. For the $H \rightarrow \gamma \gamma$ categories we use the published channel efficiencies.

Two-parameter fits were performed for each decay mode to a signal strength modifier associated with the gluon fusion (ggf) and $t \bar{t} H$ production mechanisms, $\mu_{\mathrm{ggf}+\mathrm{ttH}}$, and a signal strength modifier for the VBF and $V H$ production modes, $\mu_{\mathrm{qqH}+\mathrm{VH}}$. The results of the same fits performed with Higgs Signals are shown in Fig. 6 in direct comparison with the results from ATLAS [104,110] and CMS [77], which are faintly overlaid in the figure. Using the ATLAS results, Fig. 6a, the derived $H \rightarrow W W$ ellipse is in perfect agreement with the official result. Also the $H \rightarrow \gamma \gamma$ and $H \rightarrow Z Z$ ellipses agree reasonably well. The reproduced $H \rightarrow \gamma \gamma$ ellipse is slightly shifted towards larger values of $\mu_{\mathrm{ggf}+\mathrm{ttH}}$. A potential source of this discrepancy may be the different mass positions at which the measurements are performed. Moreover, the inclusion of correlations among the experimental systematic uncertainties becomes more important, the more the measurements are divided into smaller subsets/categories. These correlations are not publicly known and hence not taken into account by HiggsSignals. In the $H \rightarrow Z Z$ result, a significant difference between the approximations in HiggsSignals and the full profile likelihood (PLL) treatment can be observed. The PLL has a longer tail (b)

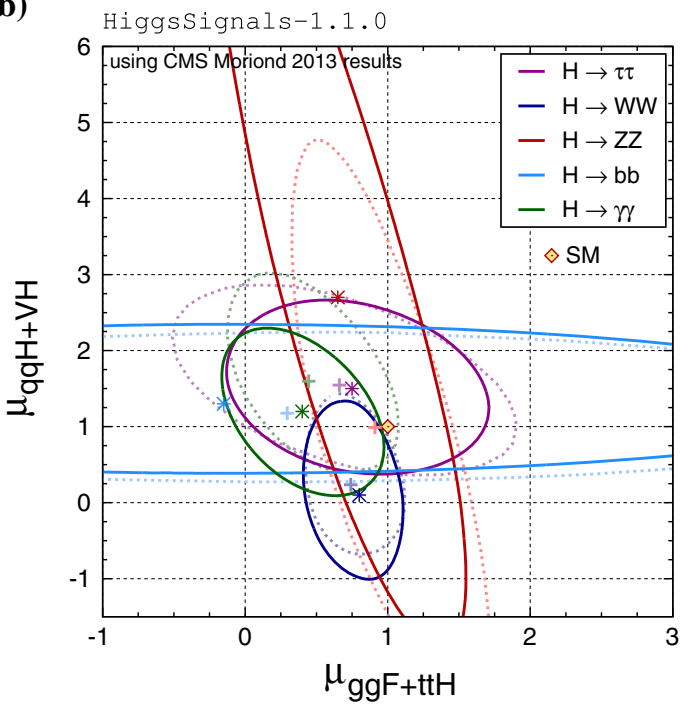

tours for the HiggsSignals results and official ATLAS/CMS result, respectively. The best fit points are given by the asterisk [plus sign] for the HiggsSignals [official] result. a Comparison with ATLAS results $[104,110]$. Both the 68 and $95 \%$ C.L. regions are shown. b Comparison with CMS results [77]. Only the $68 \%$ C.L. regions are shown

at large signal strengths, thus leading to extended 68 and $95 \%$ C.L. regions at large values of $\mu_{\mathrm{qqH}+\mathrm{VH}}$. This is partly due to the Gaussian approximation, which is more constraining at large values than a Poisson distribution with the same central value, as is used in the PLL. This is especially relevant for the very small event count for VBF $H \rightarrow Z Z$ candidates. In addition, missing information about correlations of experimental systematics might contribute to the observed difference at large $\mu_{\mathrm{qqH}+\mathrm{VH}}$. Note also that one of the two $H \rightarrow Z Z$ category measurements that are publicly available [110], cf. Table 10, is a combination of the VBF and $V H$ production channels, whereas the ATLAS analysis internally treats these channels as separate categories. The requirement of a positive probability density function (pdf) leads to the edge at negative $\mu_{\mathrm{qqH}+\mathrm{VH}}$ in the official ATLAS result. We checked that adding the requirement of a positive signal strength modifier in HiggsSignalsthis edge is reproduced quite well.

Using the CMS results, Fig. 6b, we find reasonably good agreement between HiggsSignals and the official results for $H \rightarrow W W, b b$, and $\tau \tau$. The $H \rightarrow \gamma \gamma$ ellipses roughly agree in the $\mu_{\mathrm{ggf}+\mathrm{ttH}}$ range as well as in the correlations of the fit parameters (seen in the tilt of the ellipses). However, our reproduced ellipse is shifted towards lower values of $\mu_{\mathrm{qqH}+\mathrm{VH}}$. In order to investigate the influence of correlated experimental systematic uncertainties, we introduced a tunable degree of correlation among the VBF-tagged $H \rightarrow$ $\gamma \gamma$ categories. A much better agreement between HiggsSignals and the official result is obtained when around 
$30 \%$ of the measured relative signal strength uncertainty of the VBF-tagged categories is treated as a fully correlated uncertainty. This indicates that including this type of (not public) information could potentially lead to an improvement of the HiggsSignals methodology in certain channels. A similar effect from correlations of experimental systematics may lead to the differences observed in the $H \rightarrow \tau \tau$ ellipses. The $H \rightarrow Z Z$ ellipse can only be roughly reproduced using the publicly available data for the two $H \rightarrow Z Z$ observables. Even after adjusting their production mode efficiencies, cf. Table 11, differences remain due to the Gaussian approximation and possibly further (publicly unavailable) information on the VBF-likeness of the observed signal events [82].

Using the results in Fig. 6, we can estimate the typical differences between the official results from ATLAS and CMS and the HiggsSignals implementation. We classify the difference in two ways: first, the $\Delta \chi^{2}$ in our fit between the official best fit point from the collaboration and the best fit point from HiggsSignals, and second, the distance between the two best fit points in the parameter space relative to the $1 \sigma$ uncertainty in the direction spanned by these two best-fit points.

For the comparison with the official ATLAS result, cf. Fig. 6 a, the $\Delta \chi^{2}$ is $0.158,3.5 \times 10^{-4}$ and $3.6 \times 10^{-3}$ for $H \rightarrow \gamma \gamma, H \rightarrow W W$ and $H \rightarrow Z Z$, respectively. For $H \rightarrow \gamma \gamma$ the difference is small but non-negligible, as pointed out before. The latter two can be regarded as insignificant. The difference between the best fit points of ATLAS and HiggsSignals, relative to the corresponding $1 \sigma$ uncertainty is $24,6.6$ and $7.7 \%$, respectively. Also here, a reasonable agreement well within $1 \sigma$ is observed.

For the comparison with the official CMS result, cf. Fig. $6 \mathrm{~b}$, the differences in $\chi^{2}$ between the best fit points are 0.51 for $H \rightarrow \gamma \gamma, 0.34$ for $H \rightarrow Z Z$, and less than 0.05 for the other channels. Plausible reasons for the differences in $H \rightarrow \gamma \gamma$ and $H \rightarrow Z Z$ are discussed above. For the remainder of channels there is very good agreement. The same picture arises for the relative distance of best fit points in parameter space with respect to the $1 \sigma$ uncertainty measured in the same direction, where the largest deviation is observed for $H \rightarrow \gamma \gamma$ with $44 \%$. Still, this is well within $1 \sigma$ and should be sufficient for exploratory studies of new physics models. All other channels agree significantly better.

We now turn to the discussion of global fits in the Higgs coupling scale factor benchmark scenarios. Regarding the interpretation of the following benchmark fits, it should be kept in mind that only two parameters are allowed to deviate from their SM values, while all other Higgs couplings and partial decay widths have been fixed to their SM values. The way an observed deviation from the SM manifests itself in the parameter space of coupling strength modifiers $\kappa_{i}$ will sensitively depend on how general the basis of the $\kappa_{i}$ is that one has chosen. Furthermore the framework of the coupling strength modifiers $\kappa_{i}$ as defined in Ref. [101] is designed for the analysis of relatively small deviations from the SM. In case a firm preference should be established in a parameter region that is very different from the SM case (e.g. a different relative sign of Higgs couplings), the framework of the coupling strength modifiers $\kappa_{i}$ would have to be replaced by a more general parametrization.

The first benchmark model we want to investigate is a two-dimensional fit to universal scale factors for the Higgs coupling to the massive SM vector bosons, $\kappa_{V}$, and to SM fermions, $\kappa_{F}$. In this fit it is assumed that no other modifications to the total width than those induced by the coupling scale factors $\kappa_{F}$ and $\kappa_{V}$ are present, allowing for a fit to the coupling strength modifiers individually rather than to ratios of the scale factors [101]. Note that the loop-induced effective $H \gamma \gamma$ coupling is derived in this approximation from the (scaled) tree-level couplings $H t \bar{t}$ and $H W^{+} W^{-}$and thus exhibits a non-trivial scaling behavior. In particular the interference between the $t$ and $W$ boson loops introduces a dependence on the relative sign of the scale factors $\kappa_{F}$ and $\kappa_{V}$. In the case of a relative minus sign this interference term gives a positive contribution to the $H \gamma \gamma$ coupling.

The reconstructed ATLAS and CMS fits obtained with HiggsSignals are shown in Figs. 7a and 8a, respectively. For comparison, we show the official fit results from ATLAS [104] and CMS [77] in Figs. 7b and 8b. We find overall very good agreement. The best points are located at

$\left(\kappa_{V}, \kappa_{F}\right)=\left\{\begin{array}{l}(1.12,0.85) \\ (0.88,0.95)\end{array}\right.$ with
$\chi^{2} /$ ndf $=\left\{\begin{array}{ll}34.7 / 28 & (\text { ATLAS }) \\ 19.2 / 22 & (\text { CMS })\end{array}\right.$.

The (2D) compatibility with the SM hypothesis of these points is 11.1 and $28.4 \%$ for the reproduced ATLAS and CMS fit, respectively.

In order to probe the presence of BSM physics in the Higgs boson phenomenology a fit to the loop-induced Higgs couplings to gluons, $\kappa_{g}$, and photons, $\kappa_{\gamma}$, can be performed. In this fit it is assumed that all other (tree-level) Higgs couplings are as in the SM and no new Higgs boson decay modes exist. Figures 9a and 10a show the 2D likelihood maps in the $\left(\kappa_{\gamma}, \kappa_{g}\right)$ parameter plane for the HiggsSignals result using the ATLAS and CMS observables, respectively. The corresponding official ATLAS and CMS results are given in Figs. $9 \mathrm{~b}$ and 10b. Again, we observe reasonably good agreement with the official results. We find the best fit points at

$$
\begin{aligned}
\left(\kappa_{\gamma}, \kappa_{g}\right) & =\left\{\begin{array}{l}
(1.25,1.02) \\
(0.88,0.85)
\end{array}\right. \text { with } \\
\chi^{2} / \mathrm{ndf} & = \begin{cases}34.0 / 28 & (\text { ATLAS }) \\
18.2 / 22 & (\text { CMS })\end{cases}
\end{aligned}
$$


(a)

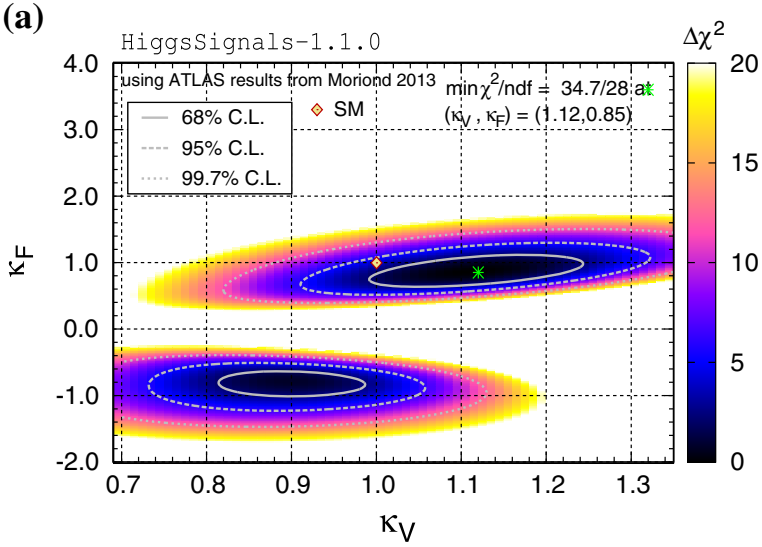

(b)



Fig. 7 Comparison of the two-parameter fits probing different coupling strength scale factors for fermions, $\kappa_{F}$, and vector bosons, $\kappa_{V}$, derived by HiggsSignals (a) and ATLAS [104] (b). The signal strength measurements used for the HiggsSignals fit are listed in Table 10. The Higgs mass is chosen to be $m_{H}=125.5 \mathrm{GeV}$
Fig. 8 Comparison of the two-parameter fits probing different coupling strength scale factors for fermions, $\kappa_{F}$, and vector bosons, $\kappa_{V}$, obtained using HiggsSignals (a) and by CMS [77] (b). The signal strength measurements used for the Higgs Signals fit are listed in Table 11. The Higgs mass is chosen to be $m_{H}=125.7 \mathrm{GeV}$ (a)

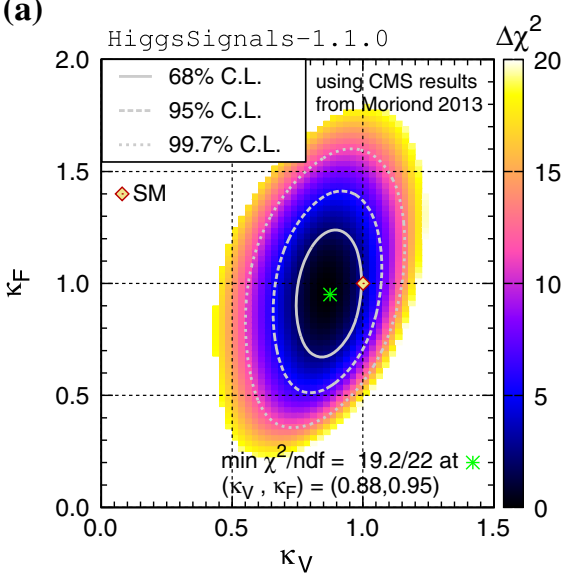

(b)

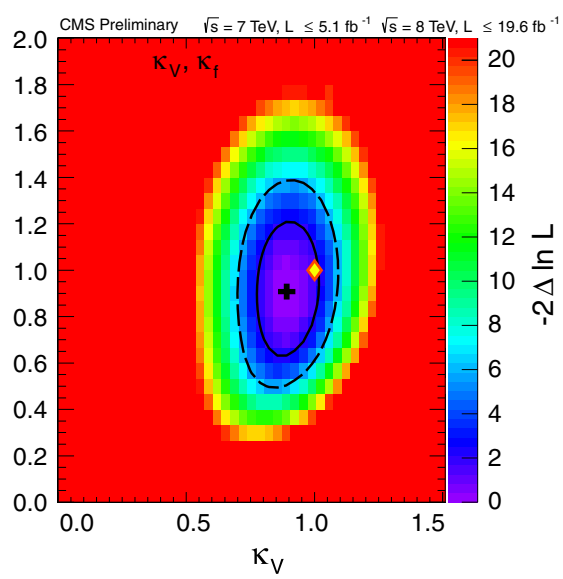

(a)

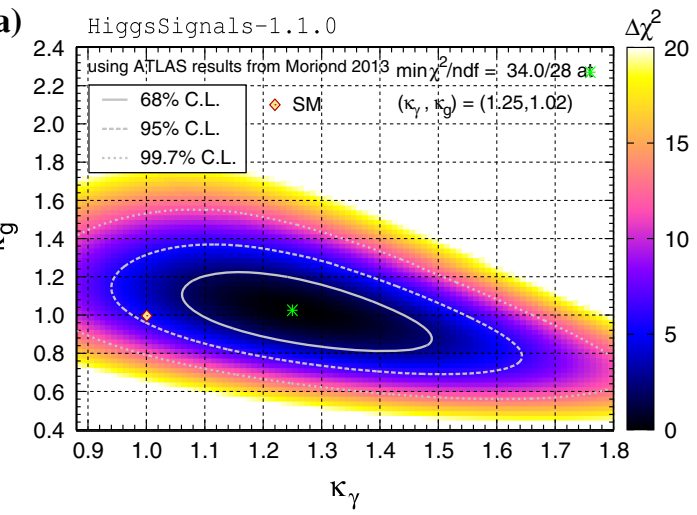

Fig. 9 Comparison of the two-parameter fits probing different coupling strength scale factors to gluons, $\kappa_{g}$, and photons, $\kappa_{\gamma}$, obtained by HiggsSignals (a) and ATLAS [104] (b). It is assumed that no new Higgs boson decay modes are open, $\Gamma_{\mathrm{BSM}}=0 \mathrm{GeV}$, and that no other

These are (2D) compatible with the SM at the level of 7.6 and $17.1 \%$, respectively. In the ATLAS fit, the best-fit region obtained by HiggsSignals is slightly shifted with respect (b)



modifications of the couplings occur with respect to their SM values. The signal strength measurements used for the HiggsSignals fit are listed in Table 10. The Higgs mass is chosen to be $m_{H}=125.5 \mathrm{GeV}$

to the official result towards lower values of $\kappa_{g}$ by roughly $\Delta \kappa_{g} \sim 0.05-0.10$, whereas the agreement in $\kappa_{\gamma}$ direction is very good. In the CMS fit, the agreement is better. Here, 


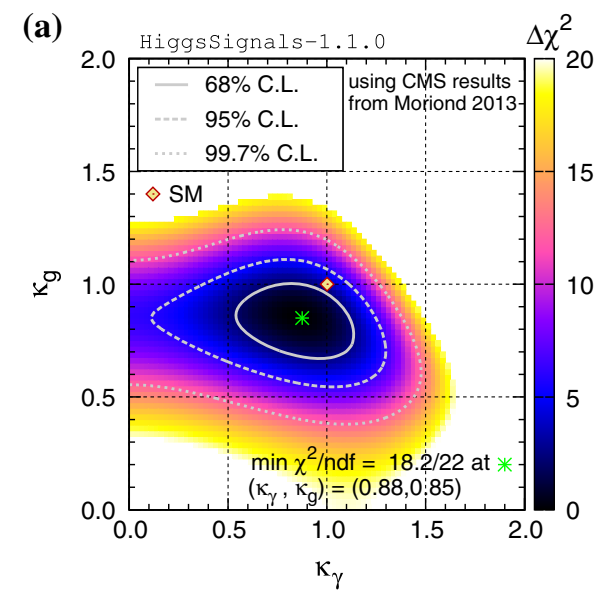

Fig. 10 Comparison of the two-parameter fits probing different coupling strength scale factors to gluons, $\kappa_{g}$, and photons, $\kappa_{\gamma}$, obtained using HiggsSignals (a) and by CMS [77] (b). It is assumed that no new Higgs boson decay modes are open, $\Gamma_{\mathrm{BSM}}=0 \mathrm{GeV}$, and that no



other modifications of the couplings occur with respect to their SM values. The signal strength measurements used for the Higgs Signals fit are listed in Table 11. The Higgs mass is chosen to be $m_{H}=125.7 \mathrm{GeV}$

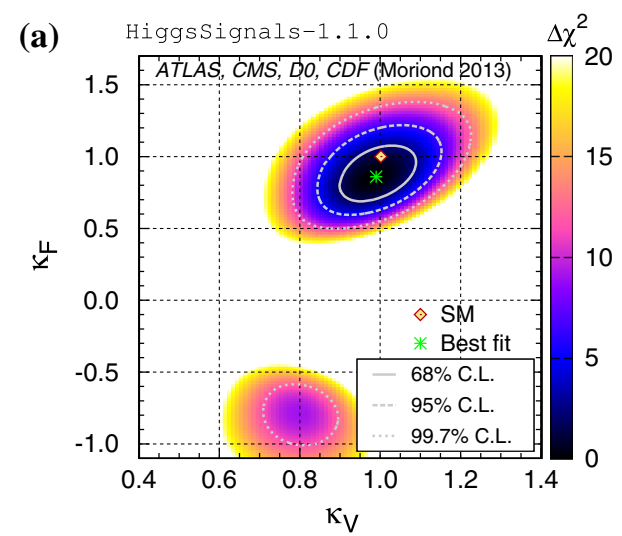

Fig. 11 Two-dimensional fit results for the two different benchmark scenarios of Higgs coupling scaling factors discussed above. a Common scale factors for the vector boson and fermion couplings, $\kappa_{V}$ and $\kappa_{F}$, respectively, b Scale factors for the loop-induced Higgs couplings

the HiggsSignals $\Delta \chi^{2}$ distribution is slightly shallower than the official CMS likelihood at low values of $\kappa_{\gamma}$, leading to slightly larger C.L. contours.

We conclude this section by pointing out that, despite some discrepancies that are observed in fits to single decay modes using subsets of the available measurements, Fig. 6, the combination of all available channels from each experiment reproduces the official results quite well. We are therefore confident that the accuracy of the HiggsSignals method is sufficient for surveys of new physics parameter spaces compatible with the Higgs measurements, and for simple coupling scale factor fits. For a more precise determination of the Higgs boson coupling structure with HiggsSignals, however, it would be desirable if the experimental collabo-

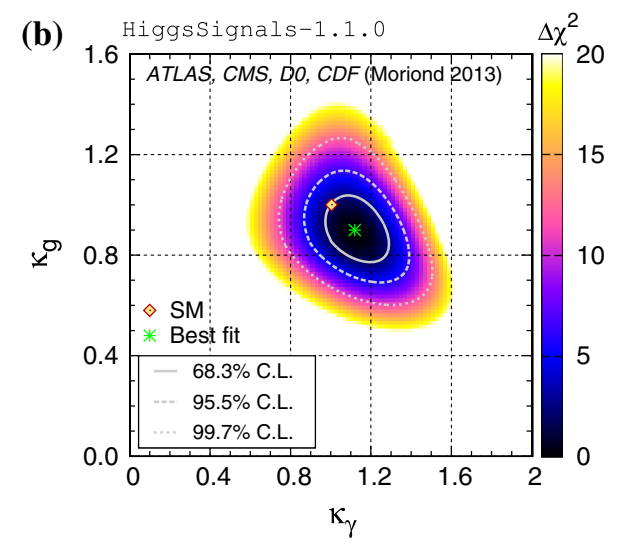

to photons, $\kappa_{\gamma}$, and gluons, $\kappa_{g}$. In these fits, the Higgs boson mass is assumed to be $126 \mathrm{GeV}$. The full available data from the Tevatron and LHC experiments as presented at the Moriond 2013 conference (and shortly after) is used. This data is summarized in Fig. 2

rations made information on efficiencies, correlated experimental uncertainties and all category measurements publicly available in a more complete way. We would expect a significant reduction of the observed remaining discrepancies if this information was included in HiggsSignals.

\subsection{Example applications of HiggsSignals}

We now go beyond validation and repeat the two discussed Higgs coupling scaling factor fits including the full presently available data from the LHC and Tevatron experiments, as listed in Fig. 2. This includes data presented up until shortly after the Moriond 2013 conference. We assume a Higgs 
boson mass of $126 \mathrm{GeV}$. The fit results for the Higgs coupling scale factors $\left(\kappa_{V}, \kappa_{F}\right)$, defined in Sect. 5.2 and [101], are shown in Fig. 11a. The best-fit point is found at

$$
\begin{gathered}
\kappa_{V}=0.99_{-0.06}^{+0.06}, \quad \kappa_{F}=0.86_{-0.10}^{+0.14}, \\
\text { with } \chi^{2} / \mathrm{ndf}=68.7 / 61,
\end{gathered}
$$

where the profiled one-dimensional $68 \%$ C.L. uncertainties are given. For this fit the SM point is found to be located well within the $68 \%$ C.L. contour, with a (2D) $\chi^{2}$ compatibility with the best fit point of $59.5 \%$. Compared to the individual results from ATLAS [104] and CMS [77] presented in Figs. 7 and 8, a significant degradation of the fit quality of the non-SM minimum (i.e. for negative $\kappa_{F}$ ) is observed, which highlights the power of such simultaneous global analyses.

A similar improvement is seen for the $\left(\kappa_{\gamma}, \kappa_{g}\right)$ fit, shown in Fig. 11b, where the best fit point is found at

$$
\begin{gathered}
\kappa_{\gamma}=1.12_{-0.08}^{+0.10}, \quad \kappa_{g}=0.90_{-0.08}^{+0.09}, \\
\text { with } \chi^{2} / \text { ndf }=67.6 / 61,
\end{gathered}
$$

which can be compared with Figs. 9 and 10. Here, the SM is compatible with the fit result at the level of $31.8 \%$. The fit shows a weak tendency towards slightly reduced $\kappa_{g}$ and slightly enhanced $\kappa_{\gamma}$. The discrimination power on $\kappa_{g}$ will increase only slowly with more data, since the large uncertainty of the rate prediction for single Higgs production is already the dominant limitation of the precision of the combined fit [45].

As a further example application we performed fits in three of the MSSM benchmark scenarios recently proposed for the interpretation of the SUSY Higgs search results at the LHC [126]. These scenarios are defined in terms of two free parameters, $\tan \beta=v_{2} / v_{1}$ (the ratio of the vacuum expectation values of the two Higgs doublets), and either $M_{A}$ (the $\mathcal{C P}$-odd Higgs boson mass) or $\mu$ (the Higgsino mass parameter). The other parameters are fixed to their default values as specified in [126] to exhibit certain features of the MSSM Higgs phenomenology. For each parameter point in these two-dimensional planes we calculated the model predictions with FeynHiggs -2.9 .4 and evaluated the total $\chi^{2}$, comprised of the LEP Higgs exclusion $\chi^{2}$ value [7,11] obtained from HiggsBounds $-4[15,16]$, as well as the total $\chi^{2}$ from HiggsSignals using the peak-centered $\chi^{2}$ method. The theoretical mass uncertainty of the lightest Higgs boson is set to $2 \mathrm{GeV}$ when treated as a Gaussian uncertainty (i.e. in the LEP exclusion $\chi^{2}$ from HiggsBounds and in Higgs Signals), and to $3 \mathrm{GeV}$ in the evaluation of $95 \%$ C.L. LHC exclusions with HiggsBounds.

The first scenario is an updated version of the well-known $m_{h}^{\max }$ benchmark scenario [126,129], where the masses of the gluino and the squarks of the first and second generation were set to higher values in view of the latest bounds from SUSY searches at the LHC, see [126] for details. The



Fig. 12 Distribution of $\Delta \chi^{2}$ in the (updated) $m_{h}^{\max }$ benchmark scenario of the MSSM [126]. The result from HiggsSignals and the LEP exclusion $\chi^{2}$ of HiggsBounds are added. The patterned areas indicate parameter regions excluded at $95 \%$ C.L. from the following LHC Higgs searches: CMS $h / H / A \rightarrow \tau \tau$ [127] (orange, checkered), ATLAS $t \rightarrow H^{+} b \rightarrow \tau^{+} v_{\tau} b$ [128] (green, coarsely striped), CMS SM Higgs combination [115] (red, striped). The $95 \%$ C.L. LEP excluded region $[7,11]$, corresponding to $\chi_{\mathrm{LEP}, \mathrm{HB}}^{2}=4.0$, is below the black dashed line. The best-fit point, $\left(M_{A}, \tan \beta\right)=(674 \mathrm{GeV}, 5.0)$ with $\chi^{2} / \mathrm{ndf}=70.2 / 66$, is indicated by a green star. The 68 and $95 \%$ C.L. preferred regions (based on the $2 \mathrm{D} \Delta \chi^{2}$ probability w.r.t. the best fit point) are shown as solid and dashed gray lines, respectively

results are shown in Fig. 12 in the $\left(M_{A}, \tan \beta\right)$ plane. Besides the colors indicating the $\Delta \chi^{2}=\chi^{2}-\chi_{\text {best-fit }}^{2}$ distribution relative to the best-fit point (shown as a green star) we also show the parameter regions that are excluded at $95 \%$ C.L. by LHC searches for a light charged Higgs boson (darkgreen, coarsely striped) [128], neutral Higgs boson(s) in the $\tau \tau$ final state (orange, checkered) [127] and the combination of SM search channels (red, striped) [115], as obtained using HiggsBounds. As an indication for the parameter regions that are $95 \%$ C.L. excluded by neutral Higgs searches at LEP $[7,11]$ we include a corresponding contour (black, dashed) for the value $\chi_{\mathrm{LEP}, \mathrm{HB}}^{2}=4.0$. Conversely, the parameter regions favored by the fit are shown as 68 and $95 \%$ C.L. regions (based on the 2D $\Delta \chi^{2}$ probability w.r.t. the best fit point) by the solid and dashed gray lines, respectively.

As can be seen in the figure, the best fit regions are obtained in a strip at relatively small values of $\tan \beta \approx 4.5-7$, where in this scenario $M_{h} \sim 125.5 \mathrm{GeV}$ is found. At larger $\tan \beta$ values the light Higgs mass in this benchmark scenario (which was designed to maximise $M_{h}$ for a given $\tan \beta$ in the region of large $M_{A}$ ) turns out to be higher than the measured mass of the observed signal, resulting in a corresponding $\chi^{2}$ penalty. At very low $\tan \beta$ values the light Higgs mass is found to be below the preferred mass region, again resulting in a $\chi^{2}$ penalty. Here, the $\chi^{2}$ steeply rises (for $M_{h} \lesssim 122 \mathrm{GeV}$ ), because the mass-sensitive observables $\left(H \rightarrow \gamma \gamma, Z Z^{(*)}\right)$ cannot be explained by the light Higgs boson anymore, cf. Sect. 5.1.1. Values of $M_{A}>300 \mathrm{GeV}$ are preferred in this scenario, and thus the light Higgs has 


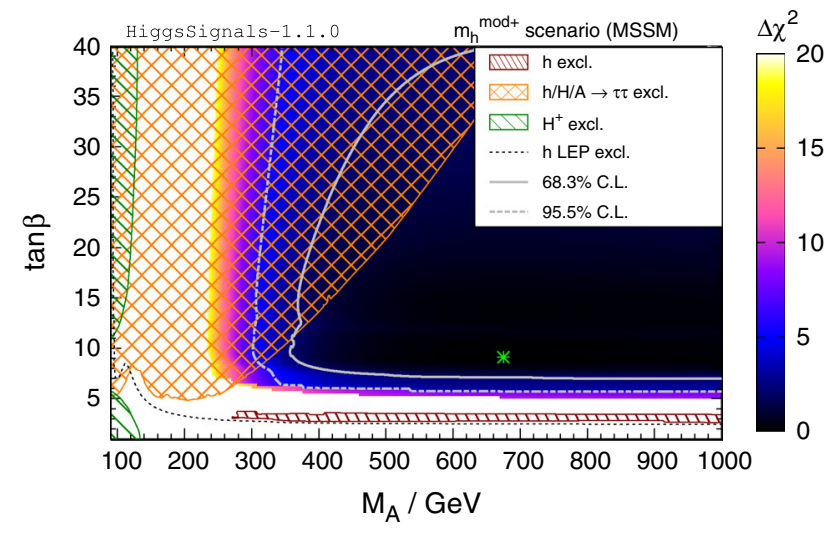

Fig. $13 \Delta \chi^{2}$ distribution (HiggsSignals and HiggsBounds LEP exclusion $\chi^{2}$ added) in the $m_{h}^{\bmod +}$ benchmark scenario of the MSSM [126]. The excluded regions and contour lines have the same meaning as in Fig. 12. The best-fit point (indicated by a green star) is found at $\left(M_{A}, \tan \beta\right)=(674 \mathrm{GeV}, 9.3)$ with $\chi^{2} /$ ndf $=70.7 / 66$

mainly SM-like couplings. Consequently, the $\chi^{2}$ contribution from the rate measurements is similar to the one for a SM Higgs boson. In this regime, the Higgs mass dependence of the total $\chi^{2}$ (from HiggsSignals) is comparable to the results shown in Fig. 3d. We find the best fit point at $\left(M_{A}, \tan \beta\right)=(674 \mathrm{GeV}, 5.0)$ with $\chi^{2} /$ ndf $=70.2 / 66$. The number of degrees of freedom (ndf) comprises 63 signal strengths and 4 mass measurements presented in Fig. 2, as well as one LEP exclusion observable from HiggsBounds.

The second scenario that we discuss here is a modification of the $m_{h}^{\max }$ scenario with a lower value of $X_{t}$, leading to $M_{h} \sim 125.5 \mathrm{GeV}$ over nearly the whole $\left(M_{A}, \tan \beta\right)$ plane [126]. This so-called $m_{h}^{\bmod +}$ scenario is shown in Fig. 13 (with the same colors and meaning of thecontours as for the $m_{h}^{\max }$ scenario, Fig. 12). The best fit point is found at $\left(M_{A}, \tan \beta\right)=(674 \mathrm{GeV}, 9.3)$ with $\chi^{2}=70.7 / 66$. Only slightly larger $\chi^{2}$ values are found over the rest of the plane, except for the lowest $M_{A}$ and $\tan \beta$ values, where $M_{h}$ is found to be below the preferred mass region. As in the preferred region for the $m_{h}^{\max }$ scenario the lightest Higgs boson is mostly SM-like here, and the $\chi^{2}$ from the rates is close to the one found in the $m_{h}^{\max }$ scenario.

As a final example, we performed a fit in the low $-M_{H}$ benchmark scenario of the MSSM [126]. This scenario is based on the assumption that the Higgs observed at $125.5 \mathrm{GeV}$ is the heavy $\mathcal{C} \mathcal{P}$-even Higgs boson of the MSSM. In this case the light $\mathcal{C P}$-even Higgs has a mass below the LEP limit for a SM Higgs boson of $114.4 \mathrm{GeV}$ [7], but is effectively decoupled from the SM gauge bosons. The other states of the Higgs spectrum are also rather light, with masses around $\sim 130 \mathrm{GeV}$, so that this scenario offers good prospects for the searches for additional Higgs bosons $[19,65,66]$. Since $M_{A}$ must be relatively small in this case the $(\mu, \tan \beta)$ plane is scanned [126], where only $\tan \beta \lesssim 10$ is considered.



Fig. $14 \Delta \chi^{2}$ distribution (HiggsSignals and HiggsBounds LEP exclusion $\chi^{2}$ added) in the low $-M_{H}$ benchmark scenario of the MSSM [126]. The excluded regions and contour lines have the same meaning as in Fig. 12, except the red, finely striped region, which gives the $95 \%$ C.L. exclusion from the CMS Higgs search $H \rightarrow Z Z^{(*)} \rightarrow 4 \ell$ [82], applied to the SM-like heavy $\mathcal{C P}$ even Higgs boson. The best-fit point (indicated by a green star) is found at $(\mu, \tan \beta)=(1850 \mathrm{GeV}, 4.9)$ with $\chi^{2} / \mathrm{ndf}=80.3 / 66$

The $\mathcal{C P}$-odd Higgs boson mass is fixed to $M_{A}=110 \mathrm{GeV}$. Our results are shown in Fig. 14. The $95 \%$ C.L. excluded regions are obtained from the same Higgs searches as in Fig. 12, except for the red patterned region, which results from applying the limit from the CMS SM Higgs search $H \rightarrow Z Z^{(*)} \rightarrow 4 \ell[82]$ to the SM-like, heavy $\mathcal{C P}$-even Higgs boson (see below).

Two distinct best-fit regions are found [126]: The parameter space with $\mu \sim(1.6-2.0) \mathrm{TeV}$ and $\tan \beta \sim 4-6$ predicts a heavy $\mathcal{C P}$-even Higgs boson with a well compatible mass value $M_{H} \approx 126 \mathrm{GeV}$ and SM-like couplings. However, large parts (at low $\tan \beta \lesssim 4$.9) of this region favored by the rate and mass measurements are severely constrained by charged Higgs searches [128]. The best-fit point is found at the edge of the excluded region at $(\mu, \tan \beta)=$ $(1850 \mathrm{GeV}, 4.9)$. The second region favored by the fit is located at large values of $\mu \sim(2.4-2.9) \mathrm{TeV}$ and $\tan \beta \sim$ $6-7$. Here, the masses of the $\mathcal{C} \mathcal{P}$-even Higgs bosons are generally lower. For instance, at $(\mu, \tan \beta) \sim(3070 \mathrm{GeV}, 6.0)$, we have $M_{h} \approx 76.1 \mathrm{GeV}$ and $M_{H} \approx 122.8 \mathrm{GeV}$. For slightly larger (lower) values of $\mu(\tan \beta)$ we find a steep edge in the HiggsSignals $\chi^{2}$ distribution, because $M_{H}$ becomes too low to allow for an assignment of the heavy $\mathcal{C} \mathcal{P}$-even Higgs boson to all mass-sensitive peak observables, cf. the results shown in Fig. 3d, Sect. 5.1.1. Due to the low mass of the light $\mathcal{C P}$-even Higgs boson in this region, the LEP channel $e^{+} e^{-} \rightarrow h A[11]$ is kinematically accessible and contributes a non-negligible $\chi^{2}$ which increases with $\mu$. The parameter space between the two preferred regions suffers a rather large $\chi^{2}$ penalty, since in particular the predicted rates for the $H \rightarrow Z Z^{(*)}, W W^{*}$ channels are above the rates measured at the LHC, as can also be seen from the $95 \%$ C.L. exclusion by HiggsBounds in this region. 
At the best-fit point we find a $\chi^{2} /$ ndf $=80.3 / 66$. Compared with the light $\mathcal{C P}$-even Higgs interpretation of the observed signal, as discussed in the $m_{h}^{\max }$ and $m_{h}^{\bmod +}$ scenarios, the fit quality is only slightly worse.

\section{Conclusions}

We have presented HiggsSignals, a public Fortran code to test the predictions of models with arbitrary Higgs sectors against measurements obtained from Higgs searches at the LHC, the Tevatron, and any potential future experiment. The code is publicly available at

\section{http://higgsbounds.hepforge.org}

The code features two statistical tests, one which determines the compatibility of the model with experimentally observed Higgs signals, and a second which tests for general compatibility with the observed Higgs data at the predicted mass(es) of the Higgs boson(s) in the theory. Since the two tests are complementary, we also provide a method to perform both simultaneously and use the combined results for models with multiple Higgs bosons.

The main experimental results used by Higgs Signals are the signal strength modifiers, $\hat{\mu}$, as a function of the Higgs mass in the various search channels. These results have to be supplemented by their respective experimental uncertainties, $\Delta \hat{\mu}$, and (preferably, if this information is available) with the experimental efficiencies and correlations. The information on $\hat{\mu}$ and $\Delta \hat{\mu}$ channel by channel constitutes the most general and robust experimental input for testing the theoretical predictions of different models, and we strongly encourage the experimental collaborations to continue to make them public with as much details provided as possible.

The default implementation of HiggsSignals uses the $\hat{\mu}$ results available from the LHC and the Tevatron, and it is planned to continuously update these results in forthcoming versions of HiggsSignals. However, it is easily possible for the user to include additional experimental data. For assessing possible future projections it is also possible to implement hypothetical future experimental results.

The input that has to be provided by the user (and which is similar to the HiggsBounds input) consists of the Higgs boson masses, preferably the corresponding theory uncertainties, the Higgs production cross sections and decay branching ratios, where several levels of approximation are possible. In case of the MSSM also the SLHA $[93,94]$ can be used as input/output format.

We presented in detail the two statistical methods provided by HiggsSignals: the peak-centered $\chi^{2}$ method, in which each observable is defined by a Higgs signal rate measured at a specific hypothetical Higgs mass, corresponding to a tentative Higgs signal. In the second, the mass-centered $\chi^{2}$ method, the $\chi^{2}$ is evaluated by comparing the signal rate measurement to the theory prediction at the Higgs mass predicted by the model. It was described how these two methods can be combined, as it is an option of HiggsSignals, to yield the most reliable consistency test. In this combination, the mass-centered $\chi^{2}$ method is applied only to those Higgs bosons which have not yet been tested with the peak-centered $\chi^{2}$ method against the same data. Similarly, in order to include a more complete set of constraints on the Higgs sector, it is recommended to use HiggsSignals together with HiggsBounds to test the model under consideration also against the existing Higgs exclusion bounds.

The installation, usage and subroutines of HiggsSignals were explained in detail, together with the various input and output formats. It was explained how the user can add new (hypothetical) experimental data. Several pre-defined example codes were presented that permit the user to get familiar with HiggsSignals and, by modifying the example codes, analyze own models of interest. As an example, by linking HiggsSignals to FeynHiggs, the consistency of any MSSM parameter point with the observed LHC signal can be analyzed in a simple way. Furthermore, some example codes demonstrate how to use HiggsBounds and HiggsSignals simultaneously in an efficient way.

We have presented several examples of the use of Higgs Signals. As a first example the combined best-fit signal strength has been determined. For the peak-centered $\chi^{2}$ method the mass dependence, the impact of correlations between the systematic uncertainties and the treatment of theoretical uncertainties has been discussed in detail. For the case of a SM-like Higgs boson, we demonstrated how the mass can be determined from a fit to the signal rate measurements as a function of the mass using the mass-centered $\chi^{2}$ method. Moreover, we employed this method for a combination of different search channels over the full investigated mass range. Various fits for coupling strength modifiers have been carried out using the peak-centered $\chi^{2}$ method. Their results have been compared for validation purposes with official results from the ATLAS and CMS collaborations, and very good agreement has been found.

It is expected that the agreement with the official results published by ATLAS and CMS could be improved even further if relative signal efficiencies of different production modes in all search channels would be publicly provided by the experimental collaborations. The same applies to a more complete description of the impact of individual experimental systematic uncertainties and their correlations amongst search channels. In particular, it would be useful if systematic uncertainties were given as a relative error on the quoted signal strength. We would furthermore welcome the publi- 
cation of the full $\hat{\mu}$ plot for every analysis to allow a $\chi^{2}$ test at various Higgs masses.

Going beyond just a validation of HiggsSignals results, we have also given a few examples of HiggsSignals applications. In particular, we have performed fits of Higgs coupling scaling factors including the full presently available data from both the LHC and the Tevatron. Furthermore we have investigated benchmark scenarios recently proposed for the SUSY Higgs search at the LHC, where we have taken into account both the limits obtained from the searches at LEP, the Tevatron and the LHC, as well as the information about the observed signal at about $126 \mathrm{GeV}$. The provided examples give only a first glimpse of the capabilities of HiggsSignals. The applicability of HiggsSignals goes far beyond those examples, and in particular it should be a useful tool for taking into account Higgs sector information in global fits.

Acknowledgments We thank Oliver Brein and Karina Williams for their great contributions to the HiggsBounds project, which was the basis for the development of HiggsSignals. We thank the Fittino collaboration, in particular Sebastian Heer, Xavier Prudent, Björn Sarrazin and Mathias Uhlenbrock, for comments and suggestions on the code development. We are grateful for helpful discussions with André David, Michael Dührssen, Michael Krämer, Stefan Liebler, Alex Read, Jana Schaarschmidt, Florian Staub and Lisa Zeune. T.S. would like to thank the Bonn-Cologne-Graduate-School for financial support and is grateful for the hospitality of the Oskar Klein Centre at Stockholm University, where part of the concepts of HiggsSignals were developed. This work is supported by the Helmholtz Alliance "Physics at the Terascale" and the Collaborative Research Center SFB676 of the DFG, "Particles, Strings, and the Early Universe". The work of S.H. was supported in part by CICYT (grant FPA 2010-22163-C02-01) and by the Spanish MICINN's Consolider-Ingenio 2010 Program under grant MultiDark CSD2009-00064. The work of O.S. is supported by the Swedish Research Council (VR) through the OKC.

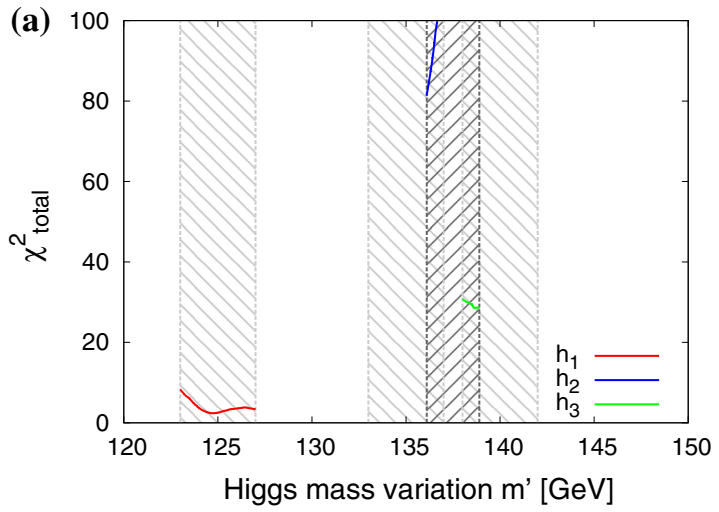

Fig. 15 Illustration of the treatment of the theoretical mass uncertainties by variation of the predicted Higgs boson masses (first option) for the toy model and observables discussed (see text). For the $H \rightarrow W W$ analysis, $h_{2}$ and $h_{3}$ are combined in a Higgs cluster $h_{23}$ with $m_{23}=$ $137.5 \mathrm{GeV}$ and $\Delta m_{23}=1.4 \mathrm{GeV}$. We show the tentative total $\chi_{i}^{2}\left(m^{\prime}\right)$ distributions for each Higgs boson $h_{i}$ for a the box-shaped and $\mathbf{b}$ the Gaussian parametrization. a Box-shaped parametrization of the theory
Open Access This article is distributed under the terms of the Creative Commons Attribution License which permits any use, distribution, and reproduction in any medium, provided the original author(s) and the source are credited.

Funded by $\mathrm{SCOAP}^{3}$ / License Version CC BY 4.0.

\section{Appendix}

7.1 Theory mass uncertainties in the mass-centered $\chi^{2}$ method

In order to illustrate the two possible treatments of theoretical mass uncertainties in the mass-centered $\chi^{2}$ method we first discuss a constructed toy example (Example 1). Then we show how a typical $\hat{\mu}$ plot changes if it is convolved with a Higgs mass pdf, which parametrizes the theoretical mass uncertainty (Example 2).

\section{Example 1: Variation of the predicted Higgs mass}

We look at a simple toy model with three neutral Higgs bosons $h_{i}(i=1,2,3)$ with masses $m_{1}=125 \mathrm{GeV}, m_{2}=$ $135 \mathrm{GeV}, m_{3}=140 \mathrm{GeV}$. For every Higgs boson the theoretical mass uncertainty is set to $2 \mathrm{GeV}$. We test this model using the experimental data from the four $\hat{\mu}$ plots of the ATLAS searches for $H \rightarrow \gamma \gamma$ [105] (7 and $8 \mathrm{TeV}$ separately), $H \rightarrow Z Z^{(*)} \rightarrow 4 \ell[121]$ and $H \rightarrow W W^{(*)} \rightarrow \ell v \ell v[120]$ (both $7+8 \mathrm{TeV}$ combination). The predicted signal strength modifiers are set for every analysis to $\mu_{1}=1.0, \mu_{2}=0.5$ and $\mu_{3}=0.2$ for the three neutral Higgs bosons, respectively. Note that the experimental mass resolution of the $H \rightarrow W W$ search is estimated to $8 \mathrm{GeV}$, while the $H \rightarrow Z Z$



mass uncertainties. The light gray striped regions show the scanned mass regions $M_{i}$ of the three Higgs bosons, whereas the darker gray striped region corresponds to $M_{k}$ of the Higgs boson cluster $k$. b Gaussian parametrization of the theory mass uncertainties. The light gray striped regions now indicate the $\chi^{2}$ contribution to the tentative total $\chi_{i}^{2}$ from the Higgs mass, cf. Eq. (19) 

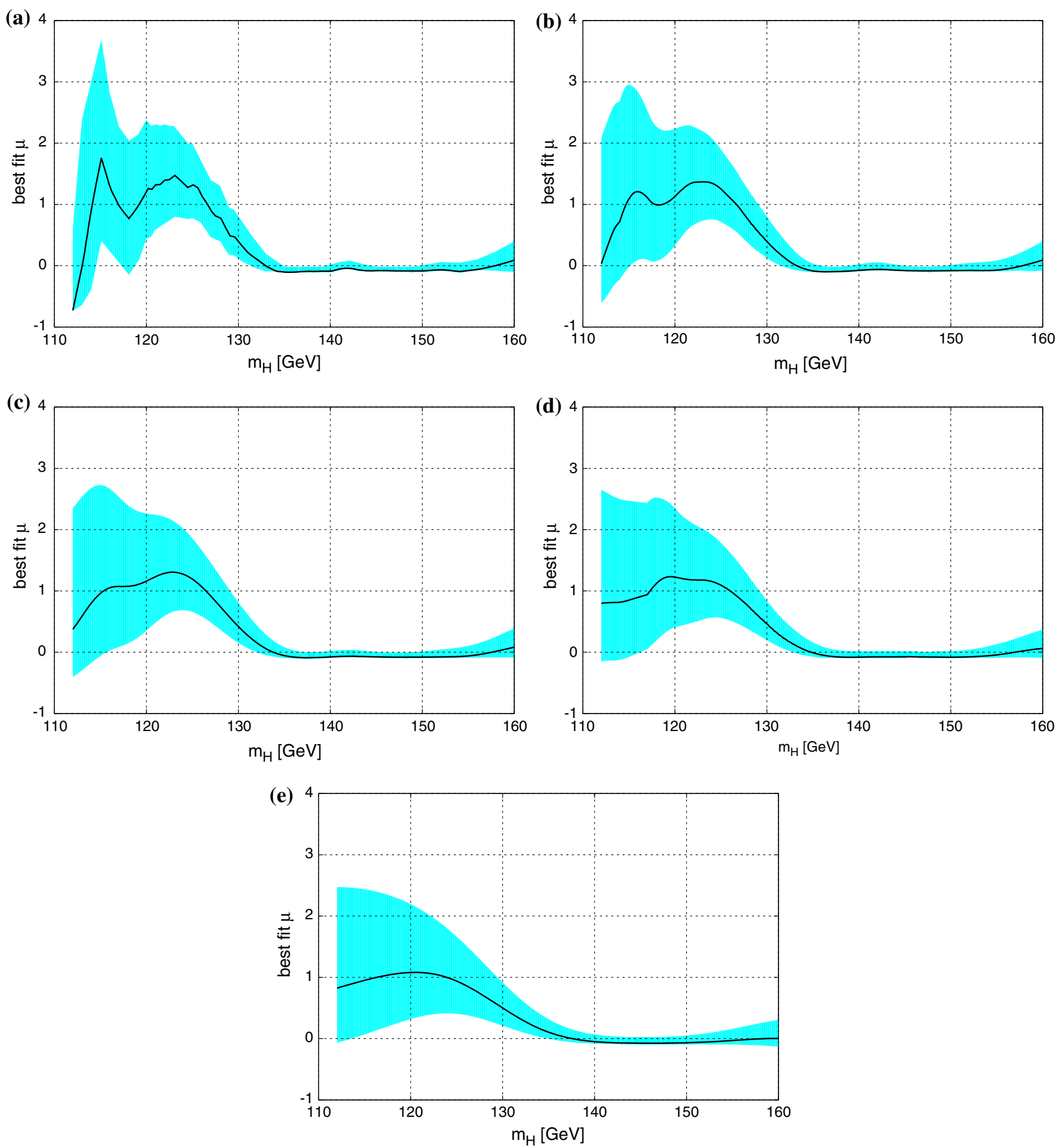

Fig. 16 Plots for the ATLAS $H \rightarrow Z Z$ analysis [121] after convolution with the Higgs mass pdf for $\Delta m=0 \mathrm{GeV}$ (a), $\Delta m=2 \mathrm{GeV}$ (b), (c) and $\Delta m=5 \mathrm{GeV}$ (d), (e), respectively. In b and $\mathbf{d}$ a uniform (box) pdf is used for the theoretical Higgs mass uncertainty, whereas a Gaussian parametrization was used in (c) and (e). a Original $\mu$ plot (from [121]) after the convolution with zero mass theory uncer-

and $H \rightarrow \gamma \gamma$ searches have a lower experimental mass uncertainty of $\lesssim 2 \mathrm{GeV}$. All $\hat{\mu}$ plots include the mass region between 120 and $150 \mathrm{GeV}$, thus all three Higgs bosons can be tested with all four analyses. tainty. $\mathbf{b} \mu$-plot after the convolution with a box-shaped mass pdf with $\Delta m=2 \mathrm{GeV}$. c $\mu$-plot after the convolution with a Gaussian mass pdf with $\Delta m=2 \mathrm{GeV}$. d $\mu$-plot after the convolution with a box-shaped mass pdf with $\Delta m=5 \mathrm{GeV}$. e $\mu$-plot after the convolution with a Gaussian mass pdf with $\Delta m=5 \mathrm{GeV}$

In the first step of the mass-centered $\chi^{2}$ method, HiggsSignals constructs possible Higgs boson combinations following the Stockholm clustering scheme. In our example, $h_{2}$ and $h_{3}$ are combined in a Higgs cluster, denoted by 
$h_{23}$, for the $H \rightarrow W W$ analysis since their mass difference is lower than the experimental mass resolution. In all other cases, the Higgs bosons are tested singly, thus we have in total 11 observables. The mass and its uncertainty associated with the Higgs cluster $h_{23}$ are derived from Eqs. (22) and (23) to $m_{23}=137.5 \mathrm{GeV}$ and $\Delta m_{23}=1.4 \mathrm{GeV}$. Its predicted signal strength is $\mu_{23}=0.7$.

In the second step, the observed quantities $\hat{\mu}_{\alpha}$ and $\Delta \hat{\mu}_{\alpha}$ have to be determined from the $\hat{\mu}$ plots for each observable $\alpha$. In order to take into account the theoretical mass uncertainties, the relevant mass region is scanned to construct the tentative total $\chi_{i}^{2}\left(m^{\prime}\right)$ distribution for each Higgs boson $h_{i}$, as described in Sect. 3.2. For this example, the $\chi_{i}^{2}\left(m^{\prime}\right)$ distributions for the box-shaped and Gaussian parametrization of the theoretical mass uncertainty are shown in Fig. 15a, b, respectively. At the mass position $\hat{m}_{i}$, where $\chi_{i}^{2}\left(m^{\prime}\right)$ is minimal, the observed quantities $\hat{\mu}_{\alpha}$ and $\Delta \hat{\mu}_{\alpha}$ are extracted from the $\hat{\mu}$ plots for those observables $\alpha$, which test the Higgs boson $i$.

In the box-shaped parametrization, the measured signal strengths of all mass-centered observables which test $h_{1}$ are defined at $\hat{m}_{1}=124.7 \mathrm{GeV}$, where $\chi_{1}^{2}$ is minimal. In contrast, the Higgs bosons $h_{2}$ and $h_{3}$ form the Higgs cluster $h_{23}$ in the $H \rightarrow W W$ analysis, therefore their allowed mass variations are restricted to the overlap regions $M_{2} \cap M_{23}$ and $M_{3} \cap M_{23}$, cf. Eq. (17), respectively. In those observables, where $h_{2}\left(h_{3}\right)$ is tested singly, the measured quantities are defined at $\hat{m}=136.1 \mathrm{GeV}(138.9 \mathrm{GeV})$. For the observable testing the Higgs cluster $h_{23}$ the observable is defined by the minimum of the joint $\chi^{2}$ distribution, which is located at $\hat{m}=138.9 \mathrm{GeV}$.

In the Gaussian parametrization the mass variation is less restricted. In contrast to the box-shaped parametrization, each mass variation is allowed over the full available mass range of the analyses, however, the additional contribution of the Higgs mass to the tentative $\chi^{2}$, cf. Eq. (19), tries to keep the varied mass close the its original predicted value. From the minimum of each tentative $\chi^{2}$ distribution, the observed quantities of analyses, which test either $h_{1}, h_{2}$ or $h_{3}$ singly, are defined at $\hat{m}=124.8,133.2$ and $140.3 \mathrm{GeV}$, respectively. For the Higgs cluster $h_{23}$ the position $\hat{m}=140.3 \mathrm{GeV}$ is chosen.

Example 2: Smearing of the $\hat{\mu}$-plot with $\Delta m$

We want to illustrate how the experimental data changes, if we choose to fold the theoretical Higgs mass uncertainty, $\Delta m$, into the original $\hat{\mu}$ plot, as discussed in Sect. 3.2. For this, we look at the $\hat{\mu}$ plot published by ATLAS for the $H \rightarrow Z Z^{(*)} \rightarrow 4 \ell$ search [121] and convolve it with a uniform (box) or Gaussian Higgs mass pdf, centered at $m_{H}$, for various theoretical mass uncertainties $\Delta m=(0,2,5) \mathrm{GeV}$, following Eqs. (20) and (21). This is done over the full mass range, $m_{H} \in[112,160] \mathrm{GeV}$, to obtain the results shown in Fig. 16. For $\Delta m=0 \mathrm{GeV}$, the $\hat{\mu}$ plot is unchanged, whereas for increasing $\Delta m$ it becomes smoother and fluctuations tend to vanish. This happens faster for the Gaussian pdf.

\section{References}

1. P.W. Higgs, Phys. Lett. 12, 132-133 (1964)

2. F. Englert, R. Brout, Phys. Rev. Lett. 13, 321-322 (1964)

3. P.W. Higgs, Phys. Rev. Lett. 13, 508-509 (1964)

4. G. Guralnik, C. Hagen, T. Kibble, Phys. Rev. Lett. 13, 585-587 (1964)

5. P.W. Higgs, Phys. Rev. 145, 1156-1163 (1966)

6. T. Kibble, Phys. Rev. 155, 1554-1561 (1967)

7. LEP Working Group for Higgs boson searches, R. Barate et al., Phys. Lett. B 565, 61-75 (2003). arXiv:hep-ex/0306033

8. TEVNPH (Tevatron New Phenomena and Higgs Working Group), CDF Collaboration, D $\varnothing$ Collaboration arXiv:1203.3774

9. TEVNPH (Tevatron New Phenomena and Higgs Working Group), CDF Collaboration, DØ Collaboration arXiv:1203.3774

10. CMS Collaboration, S. Chatrchyan et al., Phys. Lett. B 710, 26-48 (2012). arXiv: 1202.1488

11. ALEPH, DELPHI, L3 and OPAL Collaborations, S. Schael et al., Eur. Phys. J. C 47, 547-587 (2006). arXiv:hep-ex/0602042

12. ALEPH Collaboration, DELPHI Collaboration, L3 Collaboration, OPAL Collaboration, The LEP working group for Higgs boson searches, G. Abbiendi et al., Eur. Phys. J. C (2013), arXiv: 1301.6065

13. P. Bechtle, O. Brein, S. Heinemeyer, G. Weiglein, Comput. Phys. Commun. 182, 2605-2631 (2011). arXiv:1102.1898

14. P. Bechtle, O. Brein, S. Heinemeyer, G. Weiglein, K.E. Williams, Comput. Phys. Commun. 181, 138-167 (2010). arXiv:0811.4169

15. P. Bechtle, O. Brein, S. Heinemeyer, O. Stål, T. Stefaniak, et al., arXiv: 1311.0055

16. P. Bechtle, O. Brein, S. Heinemeyer, O. Stål, T. Stefaniak, G. Weiglein, K.E. Williams, arXiv:1301.2345

17. ATLAS Collaboration, G. Aad et al. Phys. Lett. B 716, 1-29 (2012). arXiv: 1207.7214

18. CMS Collaboration, S. Chatrchyan et al., Phys. Lett. B 716, 30-61 (2012). arXiv:1207.7235

19. S. Heinemeyer, O. Stål, G. Weiglein, Phys. Lett. B 710, 201-206 (2012). arXiv:1112.3026

20. P.P. Giardino, K. Kannike, M. Raidal, A. Strumia, JHEP 1206, 117 (2012). arXiv: 1203.4254

21. A. Azatov, S. Chang, N. Craig, J. Galloway, Phys. Rev. D 86, 075033 (2012). arXiv:1206.1058

22. D. Carmi, A. Falkowski, E. Kuflik, T. Volansky, arXiv:1206.4201

23. I. Low, J. Lykken, G. Shaughnessy, Phys. Rev. D 86, 093012 (2012). arXiv:1207.1093

24. T. Corbett, O. Eboli, J. Gonzalez-Fraile, M. Gonzalez-Garcia, Phys. Rev. D 86, 075013 (2012). arXiv:1207.1344

25. P.P. Giardino, K. Kannike, M. Raidal, A. Strumia, Phys. Lett. B 718, 469-474 (2012). arXiv: 1207.1347

26. J. Ellis, T. You, JHEP 1209, 123 (2012). arXiv:1207.1693

27. J. Espinosa, C. Grojean, M. Muhlleitner, M. Trott, JHEP 1212, 045 (2012). arXiv:1207.1717

28. D. Carmi, A. Falkowski, E. Kuflik, T. Volansky, J. Zupan, JHEP 1210, 196 (2012). arXiv: 1207.1718

29. S. Banerjee, S. Mukhopadhyay, B. Mukhopadhyaya, JHEP 1210, 062 (2012). arXiv: 1207.3588

30. F. Bonnet, T. Ota, M. Rauch, W. Winter, Phys. Rev. D 86, 093014 (2012). arXiv:1207.4599

31. B.A. Dobrescu, J.D. Lykken, JHEP 1302, 073 (2013). arXiv:1210. 3342 
32. G. Cacciapaglia, A. Deandrea, G.D. La Rochelle, J.-B. Flament, JHEP 1303, 029 (2013). arXiv: 1210.8120

33. T. Corbett, O. Eboli, J. Gonzalez-Fraile, M. Gonzalez-Garcia, Phys. Rev. D 87, 015022 (2013). arXiv: 1211.4580

34. E. Masso, V. Sanz, Phys. Rev. D 87, 033001 (2013). arXiv:1211. 1320

35. A. Azatov, J. Galloway, Int. J. Mod. Phys. A 28, 1330004 (2013). arXiv: 1212.1380

36. G. Belanger, B. Dumont, U. Ellwanger, J. Gunion, S. Kraml, JHEP 1302, 053 (2013). arXiv: 1212.5244

37. K. Cheung, J.S. Lee, P.-Y. Tseng, arXiv:1302.3794

38. G. Belanger, B. Dumont, U. Ellwanger, J. Gunion, S. Kraml, arXiv: 1302.5694

39. A. Falkowski, F. Riva, A. Urbano, arXiv:1303.1812

40. T. Alanne, S. Di Chiara, K. Tuominen, arXiv:1303.3615

41. P.P. Giardino, K. Kannike, I. Masina, M. Raidal, A. Strumia, arXiv: 1303.3570

42. A. Djouadi, G. Moreau, arXiv:1303.6591

43. W.-F. Chang, W.-P. Pan, F. Xu, arXiv:1303.7035

44. B. Dumont, S. Fichet, G. von Gersdorff, arXiv:1304.3369

45. A. Djouadi, arXiv: 1208.3436

46. R. Lafaye, T. Plehn, M. Rauch, D. Zerwas, M. Duhrssen, JHEP 0908, 009 (2009). arXiv:0904.3866

47. M. Klute, R. Lafaye, T. Plehn, M. Rauch, D. Zerwas, Phys. Rev. Lett. 109, 101801 (2012). arXiv:1205.2699

48. T. Plehn, M. Rauch, Europhys. Lett. 100, 11002 (2012). arXiv: 1207.6108

49. D. Carmi, A. Falkowski, E. Kuflik, T. Volansky, JHEP 1207, 136 (2012). arXiv: 1202.3144

50. A. Azatov, R. Contino, J. Galloway, JHEP 1204, 127 (2012). arXiv: 1202.3415

51. J. Espinosa, C. Grojean, M. Muhlleitner, M. Trott, JHEP 1205, 097 (2012). arXiv: 1202.3697

52. M. Montull, F. Riva, JHEP 1211, 018 (2012). arXiv: 1207.1716

53. W. Altmannshofer, S. Gori, G.D. Kribs, Phys. Rev. D 86, 115009 (2012). arXiv: 1210.2465

54. S. Chang, S.K. Kang, J.-P. Lee, K.Y. Lee, S.C. Park, et al., arXiv: 1210.3439

55. A. Celis, V. Ilisie, A. Pich, arXiv: 1302.4022

56. R. Enberg, J. Rathsman, G. Wouda, arXiv:1304.1714

57. B. Coleppa, F. Kling, S. Su, arXiv:1305.0002

58. R. Benbrik, M. Gomez, Bock, S. Heinemeyer, O. Stål, G. Weiglein, L. Zeune, Eur. Phys. J. C 72 (2012). arXiv:1207.1096

59. J.R. Espinosa, C. Grojean, V. Sanz, M. Trott, JHEP 1212, 077 (2012). arXiv:1207.7355

60. J. Cao, Z. Heng, J.M. Yang, J. Zhu JHEP 1210, 079 (2012). arXiv: 1207.3698

61. A. Arbey, M. Battaglia, A. Djouadi, F. Mahmoudi, Phys. Lett. B 720, 153-160 (2013). arXiv: 1211.4004

62. A. Arbey, M. Battaglia, F. Mahmoudi, arXiv:1303.7450

63. S. Scopel, N. Fornengo, A. Bottino, arXiv:1304.5353

64. S. Moretti, S. Munir, P. Poulose, arXiv:1305.0166

65. M. Drees, Phys. Rev. D 86, 115018 (2012). arXiv: 1210.6507

66. P. Bechtle, S. Heinemeyer, O. Stål, T. Stefaniak, G. Weiglein, L. Zeune, Eur. Phys. J. C 73, 2354 (2013). arXiv:1211.1955

67. X.-F. Han, L. Wang, J. M. Yang, J. Zhu, arXiv:1301.0090

68. J. Cao, P. Wan, J.M. Yang, J. Zhu, arXiv:1303.2426

69. P. Bechtle, K. Desch, H.K. Dreiner, M. Hamer, M. Krämer, et al., arXiv: 1310.3045

70. A. Djouadi, Phys. Rep. 457, 1-216 (2008). arXiv:hep-ph/ 0503172

71. A. Djouadi, Phys. Rep. 459, 1-241 (2008). arXiv:hep-ph/0503173

72. S. Dittmaier, M. Schumacher, Prog. Part. Nucl. Phys. 70, 1-54 (2013). arXiv:1211.4828

73. LHC Higgs Cross Section Working Group, S. Dittmaier et al., arXiv: 1101.0593
74. LHC Higgs Cross Section Working Group, S. Dittmaier et al., arXiv: 1201.3084

75. ATLAS Collaboration. ATLAS-CONF-2013-013

76. A. Denner, S. Heinemeyer, I. Puljak, D. Rebuzzi, M. Spira, Eur. Phys. J. C 71, 1753 (2011). arXiv:1107.5909

77. CMS Collaboration. CMS-PAS-HIG-13-005

78. R. Harlander, M. Kramer, M. Schumacher, arXiv:1112.3478

79. G. Cowan, K. Cranmer, E. Gross, O. Vitells, Eur. Phys. J. C 71, 1554 (2011). arXiv:1007.1727

80. S. Wilks, Ann. Math. Stat. 9, 60-62 (1938)

81. A. Wald, Trans. Am. Math. Soc. 54 (1943)

82. CMS Collaboration. CMS-PAS-HIG-13-002

83. P. Bechtle, T. Stefaniak, available online at http://higgsbounds. hepforge.org

84. CMS Collaboration. CMS-PAS-HIG-13-001

85. CMS Collaboration. CMS-PAS-HIG-12-044

86. CMS Collaboration. CMS-PAS-HIG-13-004

87. CMS Collaboration. CMS-PAS-HIG-13-003

88. S. Heinemeyer, W. Hollik, Comput. Phys. Commun. 124, 76-89 (2000). arXiv:hep-ph/9812320

89. S. Heinemeyer, W. Hollik, G. Weiglein, Eur. Phys. J. C 9, 343-366 (1999). arXiv:hep-ph/9812472

90. G. Degrassi, S. Heinemeyer, W. Hollik, P. Slavich, G. Weiglein, Eur. Phys. J. C 28, 133-143 (2003). arXiv:hep-ph/0212020

91. M. Frank, T. Hahn, S. Heinemeyer, W. Hollik, H. Rzehak et al., JHEP 0702, 047 (2007). arXiv:hep-ph/0611326

92. T. Hahn, S. Heinemeyer, W. Hollik, H. Rzehak, G. Weiglein, Comput. Phys. Commun. 180, 1426-1427 (2009)

93. P.Z. Skands, B. Allanach, H. Baer, C. Balazs, G. Belanger et al., JHEP 0407, 036 (2004). arXiv:hep-ph/0311123

94. B. Allanach, C. Balazs, G. Belanger, M. Bernhardt, F. Boudjema et al., Comput. Phys. Commun. 180, 8-25 (2009). arXiv:0801. 0045

95. M.S. Carena, S. Heinemeyer, C.E.M. Wagner, G. Weiglein, Eur. Phys. J. C 26, 601-607 (2003). arXiv:hep-ph/0202167

96. W. Porod, Comput. Phys. Commun. 153, 275-315 (2003). arXiv: hep-ph/0301101

97. W. Porod, F. Staub, Comput. Phys. Commun. 183, 2458-2469 (2012). arXiv:1104.1573

98. F. Staub, arXiv:0806.0538

99. F. Staub, Comput. Phys. Commun. 181, 1077-1086 (2010). arXiv: 0909.2863

100. F. Staub, Comput. Phys. Commun. 182, 808-833 (2011). arXiv: 1002.0840

101. LHC Higgs Cross Section Working Group, A. David et al., arXiv: 1209.0040

102. P. Uwer, arXiv:0710.2896

103. ATLAS Collaboration. ATLAS-CONF-2013-012

104. ATLAS Collaboration. ATLAS-CONF-2013-034

105. ATLAS Collaboration. ATLAS-CONF-2012-091

106. ATLAS Collaboration. ATLAS-CONF-2012-160

107. ATLAS Collaboration. ATLAS-CONF-2012-161

108. ATLAS Collaboration. ATLAS-CONF-2012-170

109. ATLAS Collaboration. ATLAS-CONF-2013-030

110. ATLAS Collaboration, G. Aad et al., Phys. Lett. B 726, 88-119 (2013). arXiv:1307.1427

111. CMS Collaboration. CMS-PAS-HIG-11-024

112. CMS Collaboration. CMS-PAS-HIG-12-042

113. CMS Collaboration. CMS-PAS-HIG-12-015

114. CMS Collaboration. CMS-PAS-HIG-12-039

115. CMS Collaboration. CMS-PAS-HIG-12-045

116. CMS Collaboration, S. Chatrchyan et al. JHEP 1305, 145 (2013). arXiv: 1303.0763

117. CDF Collaboration, T. Aaltonen et al., arXiv: 1301.6668

118. DØ Collaboration, V. M. Abazov et al., arXiv: 1303.0823 
119. ATLAS Collaboration. ATLAS-CONF-2013-014

120. ATLAS Collaboration. ATLAS-CONF-2012-098

121. ATLAS Collaboration. ATLAS-CONF-2012-092

122. ATLAS Collaboration. ATLAS-CONF-2012-168

123. ATLAS Collaboration. ATLAS-CONF-2012-127

124. CMS Collaboration. CMS-PAS-HIG-13-009

125. CMS Collaboration. CMS-PAS-HIG-12-053

126. M. Carena, S. Heinemeyer, O. Stål, C. Wagner, Eur. Phys. J. C 73, 2552 (2013). arXiv:1302.7033
127. CMS Collaboration, S. Chatrchyan et al. Phys. Lett. B 713, 68-90 (2012). arXiv: 1202.4083

128. ATLAS Collaboration, G. Aad et al., JHEP 1206, 039 (2012), arXiv: 1204.2760

129. M.S. Carena, S. Heinemeyer, C.E.M. Wagner, G. Weiglein, arXiv: hep-ph/9912223 